\title{
Triplex formation as monitored by EPR spectroscopy and molecular dynamics studies of spin -probe -labeled DNAs
}

\author{
Eva Darian \\ West Virginia University
}

Follow this and additional works at: https://researchrepository.wvu.edu/etd

\section{Recommended Citation}

Darian, Eva, "Triplex formation as monitored by EPR spectroscopy and molecular dynamics studies of spin -probe -labeled DNAs" (2002). Graduate Theses, Dissertations, and Problem Reports. 1618. https://researchrepository.wvu.edu/etd/1618

This Dissertation is protected by copyright and/or related rights. It has been brought to you by the The Research Repository @ WVU with permission from the rights-holder(s). You are free to use this Dissertation in any way that is permitted by the copyright and related rights legislation that applies to your use. For other uses you must obtain permission from the rights-holder(s) directly, unless additional rights are indicated by a Creative Commons license in the record and/ or on the work itself. This Dissertation has been accepted for inclusion in WVU Graduate Theses, Dissertations, and Problem Reports collection by an authorized administrator of The Research Repository @ WVU. For more information, please contact researchrepository@mail.wvu.edu. 


\title{
Triplex Formation as Monitored by EPR Spectroscopy and Molecular Dynamics Studies of Spin-probe Labeled DNAs
}

\author{
Eva Darian \\ Dissertation submitted to the \\ School of Pharmacy \\ at West Virginia University \\ in partial fulfillment of the requirements \\ for the degree of \\ Doctor of Philosophy \\ in \\ Pharmacy \\ Peter M. Gannett, Ph.D., Chair \\ Eugene Demchuk, Ph.D., \\ Larry E. Halliburton, Ph.D., \\ Grazyna Szklarz, Ph.D., \\ Björn Söderberg, Ph.D. \\ Department of Basic Pharmaceutical Sciences \\ Morgantown, West Virginia \\ 2002
}

Keywords: Triplex, DNA, spin labels, nitroxide, EPR, quantum mechanics, molecular dynamics 


\title{
Abstract \\ Triplex Formation as Monitored by EPR Spectroscopy and Molecular Dynamics Studies of Spin-probe Labeled DNAs.
}

\author{
Eva Darian
}

Molecular modeling has proven to be a powerful tool for studying structure and dynamics of biologically important molecules. Since the advent of nitroxide based spinprobes the electron paramagnetic resonance (EPR) study of spin-labeled macromolecules has been able to provide insight into structural and dynamics properties of DNAs, proteins, and related systems. Spin labels have been extensively used to study the dynamics of oligonucleotides. An example of this is 5-membered ring nitroxide 5-(2,2,5,5-tetramethyl-3ethynylpyrrolidine-1-oxyl)-uridine, which has been previously used in our laboratory to monitor triplex formation. Because of the difficult synthetic steps involved in the synthesis of this particular probe a new spin labeled DNA base, 5-(2,2,6,6-tetramethyl-4-ethynylpiperidyl3 -ene-1-oxyl)-uridine (6sp-uridine) is introduced in the current study. This spin label, 6sp, is readily prepared, in half the number of steps required for the previous one, and yet behaves in a spectroscopically analogous manner to its counterpart. The $6 \mathrm{sp}$ has been used here to detect the formation of a triplex DNA and to examine the relative rigidity of triplex DNA as compared to double stranded DNA using circular dichroism and EPR spectroscopy. Their EPR spectra show larger changes in response to differences in the mobility of the oligonucleotides they are attached to.

Extending their use in application to DNAs we have conducted Molecular Dynamics (MD) studies on six different oligonucleotides (ONs) molecules using the suite of programs contained in AMBER 5.0 with the Cornell force field. Quantum mechanical calculations at B3LYP level with standard 6-31G* basis set using Gaussian98 were performed. Together with available crystallographic data for different types of nitroxide molecules (Barone et al., J. Am. Chem. Soc. 1998, 120, 7069-7078), new parameters for NO- and $s p$-hybridized carbon moieties have been developed for the Cornell force field. MD simulations on single-stranded (ss), double-stranded (ds) and triple-stranded or triplex (tx) spin-probe labeled DNAs along with unmodified analogues have been studied over the course of $1 \mathrm{~ns}$. Structural and conformational properties of DNA molecules are described from the analysis of the trajectories. Dynamics of the spin-label was characterized by correlation time $\left(\tau_{c}\right)$. Our results indicate slower nitroxide motion associated with tx-DNA rather than ds- and ss-DNAs. The presence of spin labels has a substantial effect on the conformation of ss DNA, while dsand tx-DNA is not affected by the introduction of labels. We have also shown that the presence of the spin-label has small stabilizing effect on ds and tx DNAs. 


\section{Acknowledgments}

First of all I would like to express my sincerest thanks to Dr. Peter M. Gannett, my advisor, for the opportunity that he gave me in working in his laboratory. For his tremendous help in achieving my degree and enlarging my knowledge not only in the areas of synthetic and computational chemistry, but also in wide variety of spectroscopic techniques, such as NMR, EPR, and others. The large spectrum of interests that Dr. Gannett has is very impressive. It was really nice having him as advisor and hold conversations not only on the scientific level, but also at a more informal level.

Also, I would like to thank my committee members Dr. Eugene Demchuk, Dr. Larry Halliburton Dr. Grazyna Szklarz, and Dr. Björn Söderberg for finding time between their busy schedules and vacations to serve on my committee.

I sincerely thank my colleagues Jeannine Powell, Merle E. Johnson, Jonathan Daft, Sue Heavner, Spencer Ericksen. Special thanks to Jeannine and Merle for their guidance and help with the synthetic work. Sue and Spencer have helped me around the computer lab especially at the last stages of my writing.

I also would like to thank my closest friends Levon Pogossian, who became a reason of me being here in the Morgantown, Gagik Parsamian, Zara Melkoumian, and Anna Martirosyan for their professional and spiritual support.

Finally I would like to thank my husband Nelson for his patience and support toward my work. Also my parents; my mother, who put the ground for what I have become today and especially my father for his scientific impact he had in my carrier. Thanks to all my relatives, though being far away, for their encouragement. 


\section{Table of Contents}

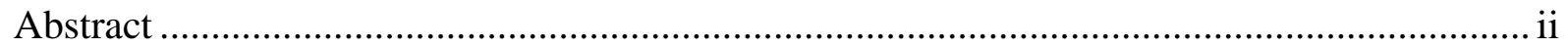

Acknowledgments............................................................................................... ii

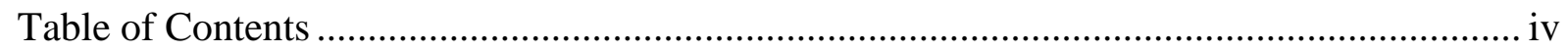

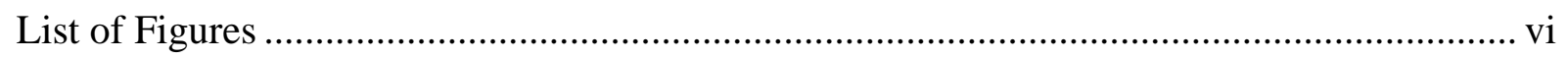

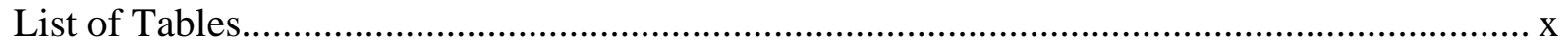

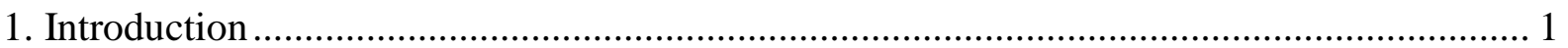

$1.1 \quad$ Background .............................................................................................. 1

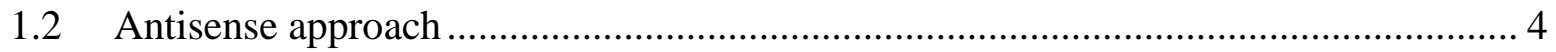

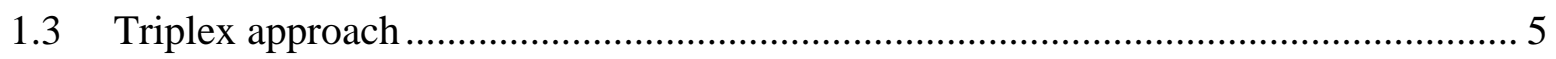

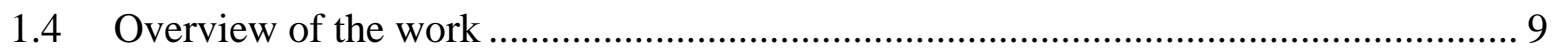

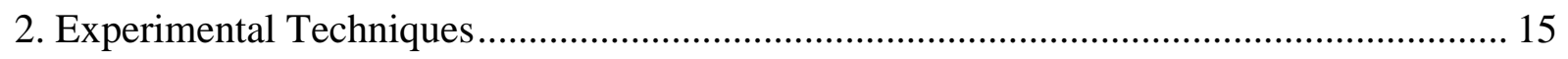

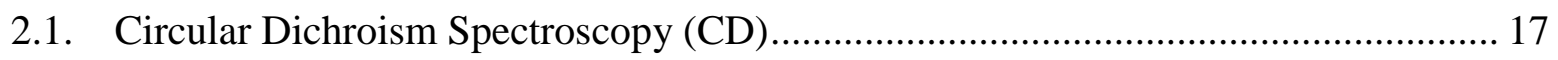

2.2. Principles of Electron Paramagnetic Resonance (EPR) .................................... 21

2.3. Nuclear Magnetic Resonance (NMR)......................................................... 27

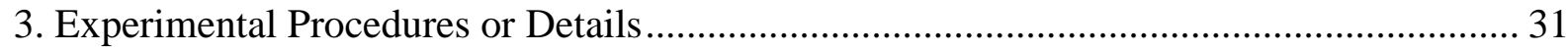

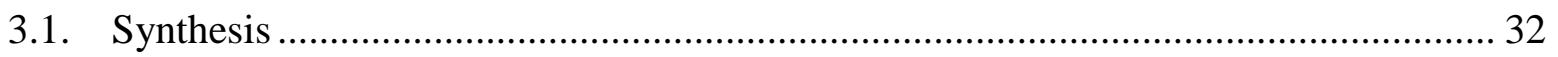

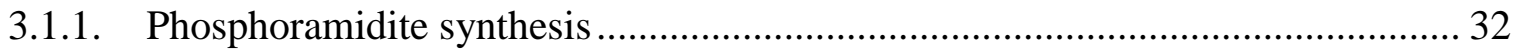

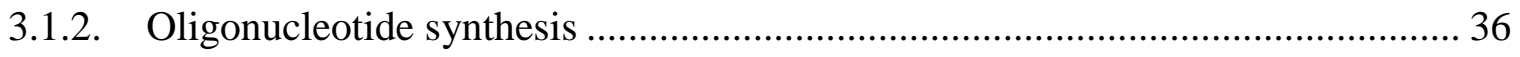

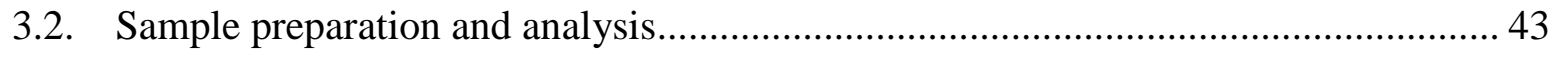

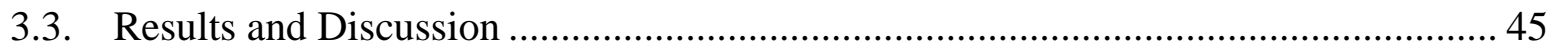

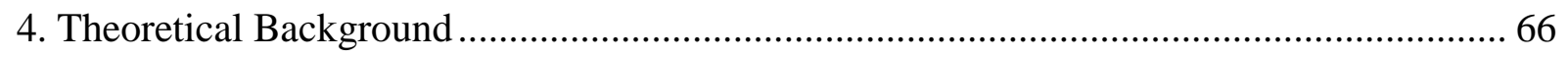

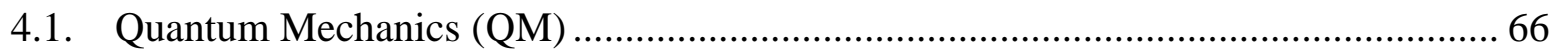




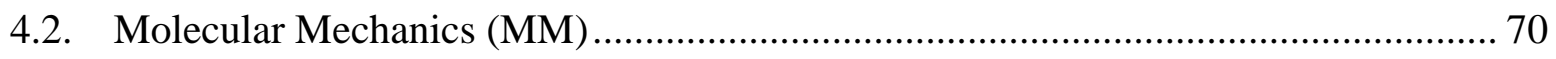

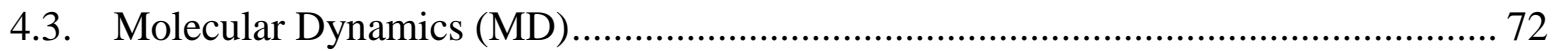

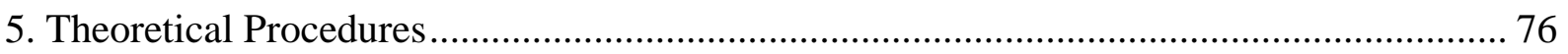

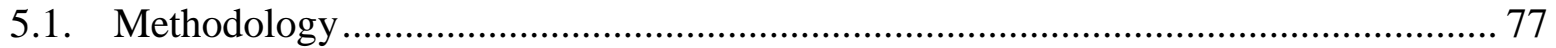

5.1.1. Development of nitroxide Force Field Parameters ...................................... 77

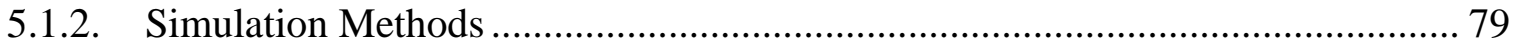

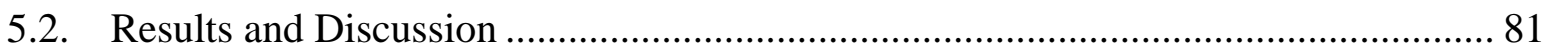

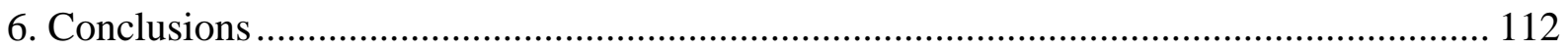

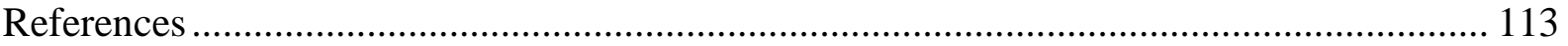

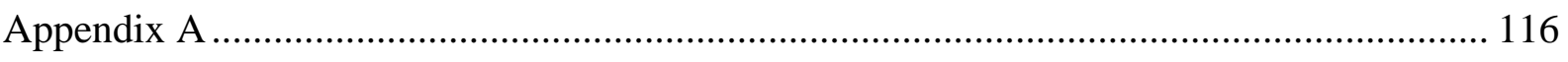

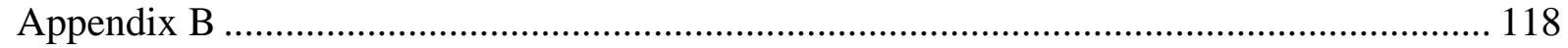




\section{List of Figures}

Figure 1.1. Double stranded DNA, single stranded mRNA, and an enzyme....................... 2

Figure 1.2. Watson-Crick base-pairing scheme for adenine (A), thymine $(T)$, guanine $(\mathrm{G})$ and cytosine $(\mathrm{C})$ bases.

Figure 1.3. Base triplet formation that follows Hoogsteen $(\mathrm{H})$ and Reverse-Hoogsteen $(\mathrm{RH})$

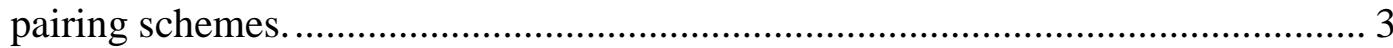

Figure 1.4. Intermolecular folding scheme illustrating the parallel and antiparallel orientation of the third strands. The homopyrimidine strand is in gray and the homopurine strand is in black. Solid line represents Watson-Crick base paring and the dashed line reverse Hoogsteen base paring.

Figure 1.5. Chemical structures of a) 5-membered ring nitroxide with acetylenic tether (5sp), b) $5 \mathrm{sp}$ phosphoramidite analogue, c) 6-membered ring nitroxide with acetylenic tether $(6 \mathrm{sp}), \mathrm{b}) 6 \mathrm{sp}$ phosphoramidite analogue.

Figure 2.1. Two-step melting of $U: A-U$ corresponding to formation of polyA;polyU and polyU at $45 \mathrm{C}$ and all single strands at 53..5 C. [After C. Stevens and G.

Felsenfeld, Biopolymers 2:293 (1964).]

Figure 2.2. a) Plane circularly polarized light resolved into its left $\mathrm{L}$ and right $\mathrm{R}$ components of similar intensities. b) Different absorbance of right (more absorbed) and left (less absorbed) components of the light result in elliptically polarized light with corresponding ellipse for the path of electric vector $\mathbf{E}$. c)

Figure 2.3. Energy level scheme as a function of the applied magnetic field $B$. $E_{1 / 2}$ and $E_{-1 / 2}$ represent the energies of $m_{S}=1 / 2$ and $m_{S}=-1 / 2$ states, respectively. $B_{r}$ is a resonant magnetic field.

Figure 2.4. Energy levels of hydrogen atom, showing hyperfine splitting. Solid lines indicate allowed transitions. For $\mathrm{A}=0$ transition is indicated with dotted line. .. 24

Figure 2.5. EPR spectra reflecting resonant field conditions $\mathrm{B}_{\mathrm{r} 1}$ and $\mathrm{B}_{\mathrm{r} 2}$. $\mathrm{A}$ is hyperfine coupling constant.

Figure 2.6. EPR spectra of nitroxide molecule at room temperature (a) and frozen sample

Figure 2.7. (a) Effects of magnetic fields on a NMR sample. A rotating frame coordinate system is defined by $x^{\prime}, y^{\prime}$, and $z$, with $z$ along $\mathbf{B}_{0}$. (b) Decay of phase coherence during precession of the magnetization in the $x$ - $y$ plane.

Figure 3.1. a) ${ }^{1} \mathrm{H}$ NMR and b) ${ }^{13} \mathrm{C}$ NMR spectra of 4-Ethynyltriisopropylsilyl-4-hydroxy2,2,6,6-tetramethyl-piperidine-1-oxyl (2). The sample was prepared in $\mathrm{CDCl}_{3}$, and reduced with phenylhydrazine. 
Figure 3.2. a) ${ }^{1} \mathrm{H}$ NMR and b) ${ }^{13} \mathrm{C}$ NMR spectra of 4-Ethynyltriisopropylsilyl-2,2,6,6tetramethyl-3,4-dehydro-piperidine-1-oxyl (3). The sample was prepared in $\mathrm{CDCl}_{3}$, and reduced with phenylhydrazine.

Figure 3.3. a) ${ }^{1} \mathrm{H}$ NMR and b) ${ }^{13} \mathrm{C}$ NMR spectra of 4-Ethynyl-2,2,6,6-tetramethyl-3,4dehydro-piperidine-1-oxyl (4). The sample was prepared in $\mathrm{CDCl}_{3}$, and reduced with phenylhydrazine.

Figure 3.4. ${ }^{1} \mathrm{H}$ NMR spectrum of 5-(2,2,6,6-tetramethyl-4-ethynylpiperidyl-3-ene-1-oxyl)uridine (5). The sample was prepared in $\mathrm{CD}_{3} \mathrm{CN}$, and reduced with phenylhydrazine.

Figure 3.5. ${ }^{1} \mathrm{H}$ NMR spectrum of 5-(2,2,6,6-tetramethyl-4-ethynylpiperidyl-3-ene-1-oxyl)5'-(4,4'-dimethoxyltriphenyl)-uridine (6). The sample was prepared in $\mathrm{CD}_{3} \mathrm{CN}$, and reduced with phenylhydrazine.

Figure 3.6. ${ }^{1} \mathrm{H}$ NMR spectrum of 5-(2,2,6,6-tetramethyl-4-ethynylpiperidyl-3-ene-1-oxyl)5'-(4,4'-dimethoxyltriphenyl)-uridine phosphoramidite (7). The sample was prepared in $\mathrm{CD}_{3} \mathrm{CN}$, and reduced with phenylhydrazine.

Figure 3.7. Synthetic scheme followed for the preparation of the new spin-label phosphoramidite 7. Reaction conditions: a) TIPS-三-Li, THF, $-78^{\circ} \mathrm{C}$, b) $\mathrm{SOCl}_{2}$, pyridine, $0^{\circ} \mathrm{C}$, c) TBAF, THF, d) $\left.\mathrm{Pd}\left[\mathrm{P}\left(\mathrm{C}_{6} \mathrm{H}_{5}\right)_{3}\right)\right]_{4}$, CuI, TEA, DMF, e) tritylchloride, TEA, $\mathrm{CH}_{2} \mathrm{Cl}_{2}$, f) $\mathrm{Cl}-\mathrm{P}\left(\mathrm{OCH}_{2} \mathrm{CH}_{2} \mathrm{CN}\right)\left(\mathrm{N}(i-\mathrm{Pr})_{2}\right)$, TEA, $\left.\mathrm{CH}_{2} \mathrm{Cl}_{2}\right)$.

Figure 3.8. Thermal denaturation curves for a) $A_{15}: T_{15}(\bullet)$, and $A_{15}:{ }^{6 s p} T_{15}(O)$ duplexes at $50 \mathrm{mM} \mathrm{MgCl} 2$ salt concentration and b) $\mathrm{T}_{15}-\mathrm{A}_{15}: \mathrm{T}_{15}(\mathbf{\square})$ and ${ }^{6 \mathrm{sp}} \mathrm{T}_{15}-\mathrm{A}_{15}: \mathrm{T}_{15}$ ( ) triplexes at $25 \mathrm{mM} \mathrm{MgCl} 2$ salt concentration.

Figure 3.9. $C D$ spectra as a function of temperature of (a) ${ }^{6 s p} T_{15}: A_{15}$ duplex and (b) $A_{15}: T_{15}$ duplex recorded from $30^{\circ} \mathrm{C}(\bullet)$ to $75^{\circ} \mathrm{C}(\boldsymbol{\square})$. All samples were prepared in $10 \mathrm{mM}$ phoshpate buffer, $\mathrm{pH} 7.4,100 \mathrm{mM} \mathrm{NaCl}$ and $50 \mathrm{mM} \mathrm{MgCl}_{2}$

Figure 3.10. CD spectra as a function of temperature of a) $\mathrm{T}_{15^{-}}-\mathrm{A}_{15}: \mathrm{T}_{15}$ triplex and b) ${ }^{6 \mathrm{sp}} \mathrm{T}_{15^{-}}$ $\mathrm{A}_{15}: \mathrm{T}_{15}$ triplex recorded from $5^{\circ} \mathrm{C}(\Delta)$ to $45^{\circ} \mathrm{C}(\bullet)$ and from $45^{\circ} \mathrm{C}(\bullet)$ to $65^{\circ} \mathrm{C}$ (ם). All samples were prepared in $10 \mathrm{mM}$ phosphate buffer, $\mathrm{pH} 7.4,100 \mathrm{mM} \mathrm{NaCl}$ and $10 \mathrm{mM} \mathrm{MgCl}_{2}$ salt concentration.

Figure 3.11. CD spectra as a function of temperature of a) $\mathrm{T}_{15^{-}} \mathrm{A}_{15}: \mathrm{T}_{15}$ triplex and (b) ${ }^{6 \mathrm{sp}} \mathrm{T}_{15^{-}}$ $\mathrm{A}_{15}: \mathrm{T}_{15}$ triplex recorded from $5^{\circ} \mathrm{C}(\Delta)$ to $45^{\circ} \mathrm{C}(\bullet)$ and from $45^{\circ} \mathrm{C}(\bullet)$ to $70^{\circ} \mathrm{C}(\square)$. All samples were prepared in $10 \mathrm{mM}$ phosphate buffer, $\mathrm{pH} 7.4,100 \mathrm{mM} \mathrm{NaCl}$ and $50 \mathrm{mM} \mathrm{MgCl} 2$. 56

Figure 3.12. EPR spectra of ${ }^{6 s p} \mathrm{~T}_{15}$ at a) $0^{\circ} \mathrm{C}$ and b) $25^{\circ} \mathrm{C}$. The solid line is the experimental spectrum and the dashed line is the simulated spectrum

Figure 3.13. EPR spectra of $\mathrm{A}_{15}:{ }^{6 \mathrm{sp}} \mathrm{T}_{15}$ at a) $0{ }^{\circ} \mathrm{C}$ and b) $25^{\circ} \mathrm{C}$. The solid line is the experimental spectrum and the dashed line is the simulated spectrum. 58 
Figure 3.14a. EPR spectra of: ${ }^{6 \mathrm{sp}} \mathrm{T}_{15}-\mathrm{A}_{15}: \mathrm{T}_{15}$ at $0^{\circ} \mathrm{C}$. Experimental and simulated spectra for both components as well as deconvoluted into fast and slow components are shown.

Figure 3.14b. EPR spectra of: ${ }^{6 \mathrm{sp}} \mathrm{T}_{15}-\mathrm{A}_{15}: \mathrm{T}_{15}$ at $25^{\circ} \mathrm{C}$. Experimental and simulated spectra for both components as well as deconvoluted into fast and slow components are shown.

Figure 5.1. Chemical structures of Dimethoxyl Nitroxide Radical (MNO), and Di(tertbutyl)aminoxyl Radical (TBNO).

Figure 5.2. Root-mean-square deviation of a) $\mathrm{A}_{15}: \mathrm{T}_{15}$ (solid), $\mathrm{A}_{15}:{ }^{5 \mathrm{sp}} \mathrm{T}_{15}$ (dotted) and $\mathrm{A}_{15}:{ }^{6 \mathrm{sp}} \mathrm{T}_{15}$ (dashed) duplex DNA and b) $\mathrm{T}_{15}-\mathrm{A}_{15}: \mathrm{T}_{15}$ (solid), ${ }^{5 \mathrm{sp}} \mathrm{T}_{15}-\mathrm{A}_{15}: \mathrm{T}_{15}$ (dotted) and ${ }^{6 s p} \mathrm{~T}_{15}-\mathrm{A}_{15}: \mathrm{T}_{15}$ (dashed) triplex DNA atoms from the initial structures as a function of time.

Figure 5.3. Root-mean-square deviation of a) $A_{15}: T_{15}$ (solid), $A_{15}:{ }^{5 \mathrm{sp}} \mathrm{T}_{15}$ (dotted) and $\mathrm{A}_{15}:{ }^{6 \mathrm{sp}} \mathrm{T}_{15}$ (dashed) duplex DNA and b) $\mathrm{T}_{15}-\mathrm{A}_{15}: \mathrm{T}_{15}$ (solid), ${ }^{5 \mathrm{sp}} \mathrm{T}_{15}-\mathrm{A}_{15}: \mathrm{T}_{15}$ (dotted) and ${ }^{6 s p} \mathrm{~T}_{15}-\mathrm{A}_{15}: \mathrm{T}_{15}$ (dashed) triplex DNA atoms to their respective mean structures as a function of time. 90

Figure 5.4. Definition of a) torsional angles and b) global groove parameters. 92

Figure 5.5. Average diameter for unmodified $(\bullet), 5 \operatorname{sp}(\square)$ and $6 \operatorname{sp}(\triangle)$ modified a) duplexes and b) triplexes.

Figure 5.6. Average backbone parameters for double stranded DNAs: $A_{15}: T_{15}(\bullet), A_{15}:{ }^{5 s} T_{15}$ (ם) and $\mathrm{A}_{15} \cdot{ }^{6 \mathrm{sp}} \mathrm{T}_{15}(\Delta)$. Bases 3-12 are the A-strand, bases 19-28 are the $* \mathrm{~T}$ strand $(*=5$ sp or $6 \mathrm{sp})$. 99

Figure 5.7. Average backbone parameters for triplex DNAs: $\mathrm{T}_{15}-\mathrm{A}_{15}: \mathrm{T}_{15}(\bullet),{ }^{5 \mathrm{sp}} \mathrm{T}_{15}-\mathrm{A}_{15}: \mathrm{T}_{15}$ (ם) and ${ }^{5 \mathrm{sp}} \mathrm{T}_{15}-\mathrm{A}_{15}: \mathrm{T}_{15}(\triangle)$. Bases 3-12 are the T-strand $(*=5$ sp or $6 \mathrm{sp})$, bases 19 28 are the A strand, and 32-43 are the T-strand. 100

Figure 5.8. Production run for $1 \mathrm{~ns}$ of $\mathrm{A}_{15}:{ }^{6 \mathrm{sp}} \mathrm{T}_{15}$ duplex DNA. Total (ETOT), kinetic (EKtot), and potential (EPtot) energies are in $\mathrm{kcal} / \mathrm{mole}$, density is in $\mathrm{g} / \mathrm{cm}^{3}$, pressure is in atmospheres, and volume in $\AA^{3}$.....

Figure 5.9. Production run for $1 \mathrm{~ns}$ of ${ }^{6 \mathrm{sp}} \mathrm{T}_{15}-\mathrm{A}_{15}: \mathrm{T}_{15}$ triplex DNA. Total (ETOT), kinetic (EKtot), and potential (EPtot) energies are in $\mathrm{kcal} / \mathrm{mole}$, density is in $\mathrm{g} / \mathrm{cm}^{3}$, pressure is in atmospheres, and volume in $\AA^{3}$.....

Figure 5.10. Definition of the magnetic axis coordinate system for calculation of rotational diffusion coefficients. 
Figure 5.11. Selected backbone torsional parameters for $5 \mathrm{sp}$ modified ss DNA. A) and of the $5^{\text {th }}$ base, B) of the $7^{\text {th }}$ base. Dotted plot represents unconstrained dynamics over $1 \mathrm{~ns}$, solid line represents constrained dynamics over $650 \mathrm{ps}$ run.

109

Figure 5.12. $5 \mathrm{sp}$ modified ss DNA snapshots at different points during the $1 \mathrm{~ns}$ run. 


\section{List of Tables}

Table 3.1. UV melting temperatures for the unmodified and spin-labeled modified duplexes and triplexes.

Table 3.2. Correlation times for single stranded, double stranded and triple stranded DNAs containing $\mathrm{T}^{6 \mathrm{sp}}$ spin labels. For two component fits, relative amounts in \% of both species present are also listed....

Table 5.1. Geometrical parameters for a) Dimethyl Nitroxide Radical (DMNO) and b) Di(tert-butyl)aminoxyl Radical (TBNO). 82

Table 5.2. Geometrical parameters for $5 \mathrm{sp}$ and $6 \mathrm{sp}$ nitroxide molecues

Table 5.3. Partial electronic charges of $5 \mathrm{sp}$ and $6 \mathrm{sp}$ fragments of modified thymidine evaluated by fitting the $6-31 \mathrm{G}^{*}$ electrostatic potentials with the RESP algorithm. 85

Table 5.4. Partial electronic charges of thymidine fragment DT from Amber compared to the thymidine fragment of modified thymidine DTMevaluated by fitting the $6-31 \mathrm{G}^{*}$ electrostatic potentials with the RESP algorithm. 86

Table 5.5. Average torsion angles for canonical DNA helices (in ${ }^{\circ}$ )

Table 5.6a. Global groove width parameters for unmodified and 5sp and 6sp modified DUPLEXES.

Table 5.6b. Global groove depth parameters for unmodified and 5sp and 6sp modified DUPLEXES.

Table 5.7a. Global groove width parameters for unmodified and 5sp and 6sp modified TRIPLEXES.

Table 5.7b. Global groove depth parameters for unmodified and $5 \mathrm{sp}$ and $6 \mathrm{sp}$ modified TRIPLEXES

Table 5.8. Diameter for unmodified and $5 \mathrm{sp}$ and $6 \mathrm{sp}$ modified DUPLEXES and TRIPLEXES.

Table 5.9. Global axis parameters for unmodified and $5 \mathrm{sp}$ and $6 \mathrm{sp}$ modified ds and tx DNAs.

Table 5.10. Internal energies, solvation free energies calculated with GB method, and a total energies of all duplexes and triplexes. All energies are calculated from 250 structures over $1000 \mathrm{ps}$ and the units of energy are kcal/mol. Standard deviations are presented in parenthesis (italic). 
Table 5.11. Rotational diffusion coefficients and correlation times calculated for $5 \mathrm{sp}$ modified ss DNA, and 5sp and 6sp modified ds and tx DNAs. Diffusion coefficients are in $10^{10} \mathrm{~s}^{-1}$

Table 5.12. Global axis parameters for unmodified and $5 \mathrm{sp}$ modified ss DNA. 110 


\section{CHPTER 1}

\section{Introduction}

\subsection{Background}

The conventional Watson-Crick double helix has lost its symmetry because of the appearance of local perturbations caused by base modification, mispairing, bulges, etc. An ever-growing collection of new structures including triple and quadruple helices has joined the standard duplex. Several decades ago it was shown that single stranded oligonucleotides can spontaneously interact with Watson-Crick duplexes to form triplex DNA structures ${ }^{1}$. Triplex DNA did not receive much attention then, however, the interest in triplex DNA structures has increased substantially very recently. This interest is enhanced mostly with the advent of technology and science and because of the potential biological role of triplex forming oligonucleotides (TFOs) in binding to target duplexes for experimental, therapeutic and diagnostic purposes. The demand for developing new techniques for describing and analyzing the new structures arises from this increasing complexity of DNA structure and conformation.

Much of the drive to understand nucleic acid interactions has come from the interest in understanding the mode of action of existing medicinal agents and from the desire to develop a new generation of superior drugs. Few drugs have been designed from a basic understanding of the biochemical mechanisms that are responsible for the disease stage. Besides, most drugs are designed to act at a level of the enzymes, which also tends to decrease their effectiveness. Since the DNA and messenger RNA (mRNA) are the key species involved in the enzyme production, it would be more efficient to design drugs that could act directly on either of them. Figure 1.1 illustrates the possible targets. 


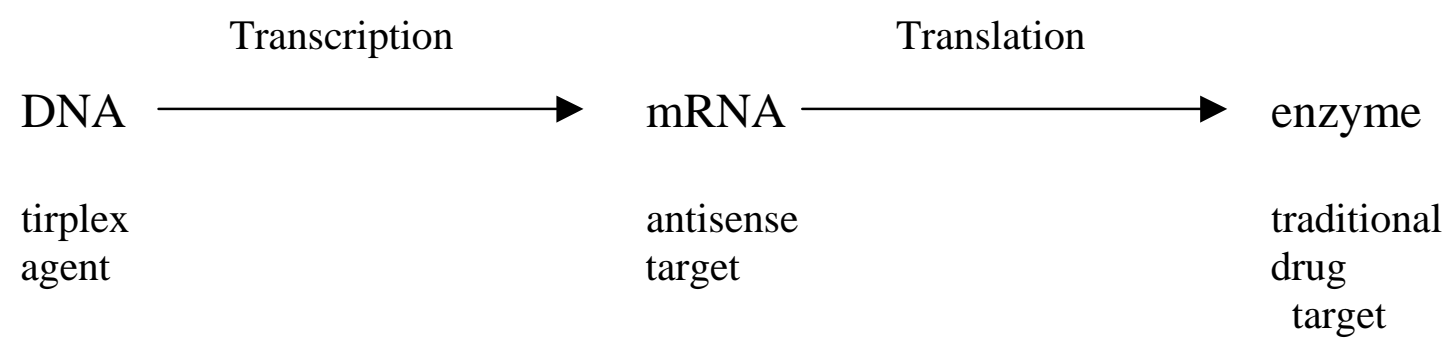

Figure 1.1. Double stranded DNA, single stranded mRNA, and an enzyme.

One way to improve drugs is to target mRNA with the single-stranded DNA (ss DNA) thus forming a DNA-RNA hybrid. The binding of the two strands follows Watson-Crick base pairing rules, where purines $(\mathrm{Pu})$ adenine and guanine $(\mathrm{A}$ and $\mathrm{G})$ pair with pyrimidines $(\mathrm{Py})$ thymine and cytosine ( $\mathrm{T}$ and $\mathrm{C}$ ) by forming two and three hydrogen bonds between $\mathrm{A}: \mathrm{T}$ and G:C bases, respectively as shown in Figure 1.2. This kind of hybrid formation can lead to inactivation of the complementary mRNA sequence.

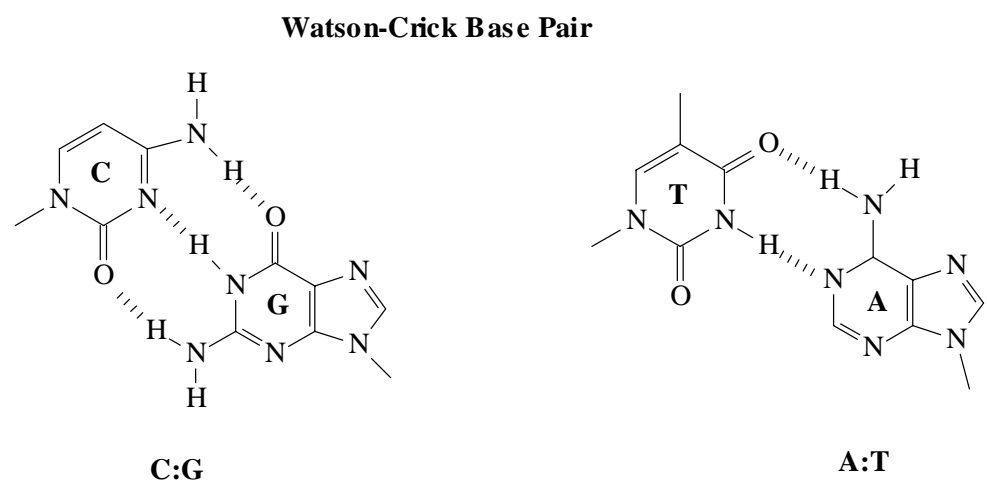

Figure 1.2. Watson-Crick base-pairing scheme for adenine (A), thymine $(T)$, guanine $(G)$ and cytosine $(\mathrm{C})$ bases.

The second, and even more efficient way, is to block the double-stranded DNA (ds DNA) with another ss DNA by forming triple-stranded DNA complex (tx DNA) or simply triplex. This, for example, could prevent DNA from producing more mRNA, binding of the 
regulatory protein to ds DNA, or could result in inhibition of oncogene such as c-myc transcription in vivo. The requirements for triplex formation are more restricted, because it must follow the Hoogsteen base pairing rules as illustrated in Figure 1.2. This means, that only certain and fewer base pairing combinations are possible. The resulting base-triplets form the core of a triple helix, where $\mathrm{C}^{+}$is the $\mathrm{N}-3$ protonated form of cytosine.

\section{Hoogsteen Base pair}

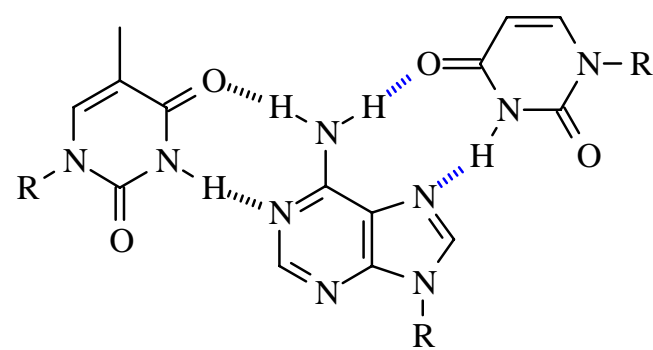

T:A-T

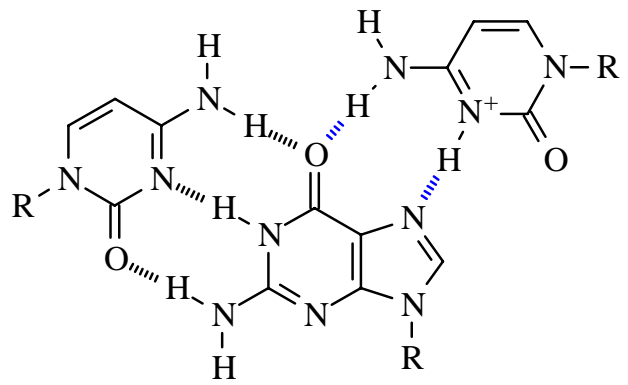

C:G-C ${ }^{+}$

Reverse Hoogsteen Base pair

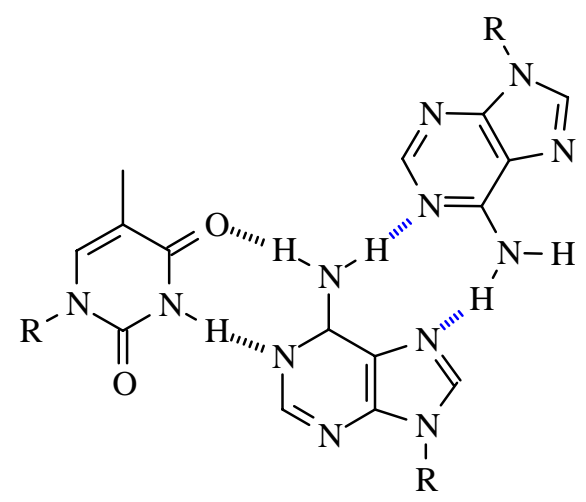

T:A-A

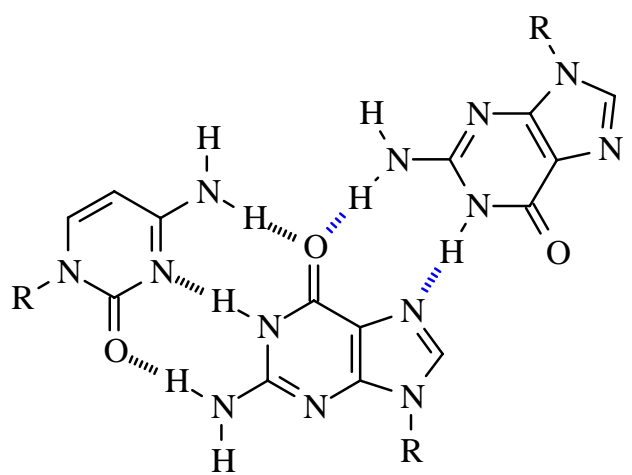

C:G-G

Figure 1.3. Base triplet formation that follows Hoogsteen $(\mathrm{H})$ and Reverse-Hoogsteen $(\mathrm{RH})$ pairing schemes. 
Analysis of eukaryotic DNA sequence databases reveals the widespread occurrence of polypurine-polypyrimidine tracts, many with the potential for triplex formation, thus having both biological function as well as therapeutic significance. Because of the third strand base pairing rules, a polypurine-polypyrimidine region will define a unique third strand pairing sequence. Given a unique sequence of DNA, it is possible to design therapeutic oligonucleotides (ONs) that will specifically bind to it and to inhibit gene expression. Development of new therapies based on ONs is at the stage of intense research. During the past 15 years, ONs have been increasingly used to inhibit gene expression and, as suggested above, there are two basic approaches to implementing ON-based therapy: an antisense and a triplex (or antigene) therapies.

\subsection{Antisense approach}

Over the past few years there have been many advances in the design and characterization of antisense ONs for the treatment of various gene based human diseases such as acquired immune disease (AIDs). In this approach, designed ONs are complementary to key targets in mRNA, which are synthesized on a DNA template in a process known as DNA transcription. DNA carries the information for protein synthesis, as well as transfer (tRNA), ribosomal (rRNA) and other RNA molecules that have structural and catalytic activities. Depending on the target site, the antisense oligonucleotide can act to inhibit translation (cap, activator protein binding site or AUG, and the sequence nucleotides that initiate peptide synthesis), to block splicing (splice donor or acceptor site) or to induce cleavage by endogenous Rnase $\mathrm{H}$, which cleaves RNA-DNA hybrids. Note that an oligonucleotide strand that is longer than the target sequence could fold back into hairpin and 
form a triplex at a target sequence. Such hairpin could form an especially stable and specific clamp to inhibit translation.

To be effective as an antisense drug an ON must possess a number of properties such as nuclease resistance, stable and specific complex formation with the target mRNA and cellular uptake. The promise of controlling gene expression in a specific and efficient manner has spurred a large research effort to develop antisense DNA therapy. At the same time this strategy has its limitations. These include poor absorption through cellular membranes, low affinity for the target mRNA sequences, and degradation by naturally occurring nuclease enzymes. Endonucleases, for example, break the phosphodiester bond at any point along the DNA chain. They can nick and digest covalently closed circular DNA and can introduce breaks internally within a linear DNA molecule. In trying to solve these problems synthetic modifications of DNA backbone by conversion of phosphates to phosphorothioate or methyl phosphonate and base modifications have been proposed. These methods can impart nuclease resistance and simultaneously improve cellular absorption. In order to bind to their target, ONs must be designed within Watson-Crick complementary sequence, which in turn provides the selectivity for the desired target. Selectivity depends also on base sequence and ONs length. Almost complete selectivity with the human genome is predicted for ONs 20-30 bases in length. For shorter chains, the chances of a given sequence occurring more than once in a mRNA are increased hence selectivity may be decreased.

\subsection{Triplex approach}

Interest in triple-helical conformations has been stimulated by several recent

discoveries. First, in 1987, Mirkin et $a l^{2,3}$ have shown that an intermolecular triplex could 
form within a single homopurine-homopyrimidine duplex DNA in supercoiled DNA. This observation reawakened a general interest in triplex DNA because many sequences in the human genome have the potential to form intermolecular triplex structures that are associated with regulatory regions of genes. Then, Frank-Kamenetskii and co-workers demonstrated that in addition to a continuous strand of purine bases, the homopurine-homopyrimidine region must contain mirror repeat symmetry ${ }^{2},{ }^{4}$. Mirror repeat is a region of DNA that has the same base sequence reading in both the $3^{\prime}$ and the $5^{\prime}$ direction from a central point in one strand of DNA. For example, a sequence like GAAGGAAG, can represent a mirror repeat. These sequences could form both parallel and anti-parallel triplex by dissociation of half of the mirror repeat duplex followed by triplex formation between the free pyrimidine strand and the remaining half of the mirror repeat (Figure 1.4).
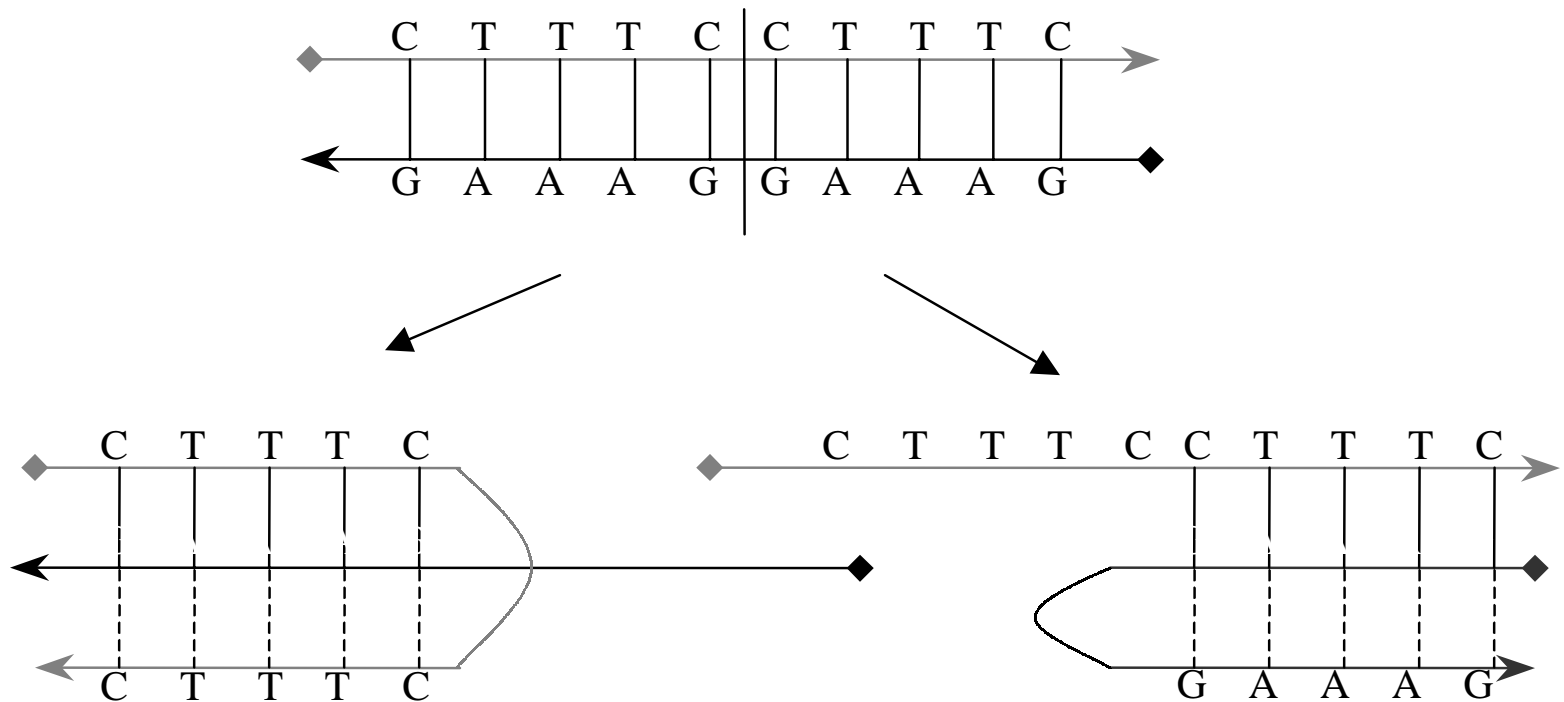

Figure 1.4. Intermolecular folding scheme illustrating the parallel and antiparallel orientation of the third strands. The homopyrimidine strand is in gray and the homopurine strand is in black. Solid line represents Watson-Crick base paring and the dashed line reverse Hoogsteen base paring. 
Such conformations could occur in cells in similar regions and may represent a gene control mechanism. In other words, what was shown was the involvement of triplex DNA formation in cellular regulation.

The discovery of the potential for triplexes to affect gene expression lead to the antigene therapy and this may become a crucial step for treatment of gene based diseases such as human immunodeficiency virus (HIV). The triplex-forming oligonucleotides (TFO) can be prepared that target specific sites on naturally occurring genes and this kind of formation can disrupt the copying of the genetic material into messenger RNA, the first step in protein synthesis. Within the past seven years triplex DNA has turned out to have therapeutic potential as well.

Triplex DNA formation must follow specific rules that are similar to those for ds DNA. Thus, there are specific motifs that allow for the formation of DNA triplets as it was shown in Figure 1.3. As mentioned earlier, there are four different ways in which homopurine-homopyrimidine mirror repeat sequences can fold into an intermolecular triplex. The most common structures are $P y-\mathrm{Pu}: \mathrm{Py}$ and $P u$-Pu:Py configurations (eg. T:A-T or C:G$C+$ and $\mathrm{C}: \mathrm{G}-G$ or T:A-A) where A:T and G:C corresponds to a normal ds DNA Watson-Crick base pairing and $T$ or $C+$ and $G$ or $A$ are the third bases that associate with ds DNA through hydrogen bonding interactions in Hoogsteen and reverse Hoogsteen base pairing fashion, respectively. With this knowledge, an oligonucleotide sequence, capable of forming a tx DNA complex can be designed to target a specific sequence in a ds DNA molecule. Moreover, since gene-based diseases like AIDs have DNA sequences that are unique to the virus that causes them, an ON can be designed to bind to the viral genomic DNA sequence and thereby inhibit selected processes in a diseased cell, to terminate the result of the disease 
process, or simply kill the cell. A sufficiently long DNA strand can bind with very high selectivity to a desired duplex gene. Also, additional agents to cross-link or cleave the genomic DNA could be attached to TFOs to permanently inactivate the gene with minimal or no toxic side effects.

There are several difficulties that are hampering the development of the antigene or triplex approach. First, although triplexes can be formed with high specificity, they generally have lower stability than duplexes under typical physiological conditions. Second, while there are many methods available for studying tx DNA in vitro, including thermal denaturation, circular dichroism (CD), X-ray crystallography, NMR and molecular modeling, few of these methods can be used in vivo. Currently, there is no direct methodology available for studying the delivery of antisense or triplex oligonucleotides to their targets directly in cellular systems, and only indirect methods can be used. Thus, the amount of information that can be obtained from more complex molecular systems is limited. Since the ON sequence was designed to be complementary to specific target, then in cells it has been assumed that binding to the intended target occurs. However, there are some examples that suggest the binding of non-sense ONs to target sequences. A non-sense sequence is a random sequence of bases non-complementary to mRNA sequence, and would not be predicted to bind based on Hoogsteen base-pairing schemes to their target.

A major search is under way to find small molecules that can stabilize triplex formation selectively. Triple helix stability can be enhanced by the use of modified nucleotides 5 . For example, 5-methylcytosine increases stability at neutral $\mathrm{pH}$, due to its hydrophobic effect, and 5-bromouracil can replace thymine. Probes are needed that can directly observe the binding of antisense or triplex ONs in cellular systems and that 
distinguish a non-specific response of cells from a specific one (i.e. DNA-mRNA or tx DNA formation) due to binding. Moreover, the existence of such a probe would be useful for examining processes like cellular uptake of ONs and their migration into the cellular nucleus.

\subsection{Overview of the work}

We are interested in using spin-probe labels to determine local base dynamics and to monitor ds- and tx-DNA formation. Structural and dynamic properties of these molecules can be obtained by one of two methods 1) experimentally, by mostly X-ray crystallography, NMR, CD spectroscopic studies etc., 2) theoretically, using molecular dynamics (MD) simulations and quantum mechanical computations. In the current work, both experimental and computational approaches are considered. To address some of the major problems hampering the progress and development of oligonucleotide based therapies and provide a tool to probe the binding, structure, dynamics and stability of antisense and triplex forming ONs upon binding to their targets, the spin probe technique is introduced. Spin labels are kinetically stable nitroxide radicals, often generated by the reaction between spin traps (nitroso compounds or nitrones) and short-lived free radicals. Since they possess an unpaired electron, they can be observed by electron paramagnetic resonance (EPR).

EPR spectroscopy has been used to analyze the conformational, dynamic, and other structural properties of spin labeled species such as DNAs, RNAs, proteins, membranes, and

DNA-protein complexes. ${ }^{6-8} 9$. In addition, many biological molecules are diamagnetic and therefore the EPR signal of the radical attached to a biopolymer can be easily distinguished from the background. 
Nitroxide based spin-probes have been used to study protein structure and proteinsubstrate interactions. Spin-labeled DNAs, based on proxyl 5-membered ring nitroxides have been intensively studied for many years to probe the conformational mobility and other structural and dynamics properties of oligonucleotides. ${ }^{10,11}$. Considerable information can be obtained about the nuclei in the immediate neighborhood of the absorbing spin, and sometimes relaxation-time data detect long-range effects. Diffusion constants, correlation times, and the type of hydration can be determined from EPR spectra of solutions. Spin labels are sensitive to the environment they are in and that sensitivity is reflected in their motion and thereby, in an EPR signal. The EPR spectra range from isotropic, reflecting more or less unconstrained spin labeled oligonucleotides, to anisotropic, as the spin labeled oligonucleotide becomes more constrained. Thus, by monitoring the EPR spectrum of a spin labeled oligonucleotide it is possible to observe the formation of a variety of DNA structures such as loops and double stranded DNA. ${ }^{12},{ }^{13},{ }^{14}$. Motional effects on nitroxide spectra can be analyzed by computer simulation of the spectra in the slow tumbling region and can be quantified through the information about correlation time, anisotropy of the motion and the local ordering of the probe. ${ }^{15}, 16$.

The tether length by which nitroxides are attached to the oligonucleotides base or backbone, for example, dictates the appearance of EPR spectra. Spin labels that are attached to DNA or RNA bases by a couple of single bonds or to the backbone, through covalent modification of phosphorothioate, make the tether pretty flexible. This type of modification produces relatively small differences between single and double stranded DNA states ${ }^{17}$. Spin labels that are more rigidly attached to the oligonucleotide, for example, via an acetylenic tether (5sp) (Figure 1.5a) as demonstrated by Spaltenstein et al, show large changes in their 
EPR spectra between the unbound and bound spectra. ${ }^{18,19}$. It has been also suggested that acetylenic modifications enhance duplex formation as well as increase the stability of triple helical complexes due to increased stacking in the third strand and triple helix junctions ${ }^{20,21}$. By synthesizing a spin labeled thymidine analog phosphoramidite, ${ }^{{ }^{\mathbf{s p}} \mathbf{T}}$ (Figure 1.5b) and preparing several oligonucleotides containing this spin label, Spaltenstein and coworkers have shown how useful they were for detecting and distinguishing between various DNA structures.

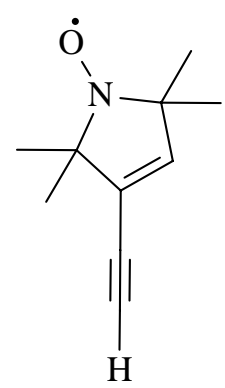

a

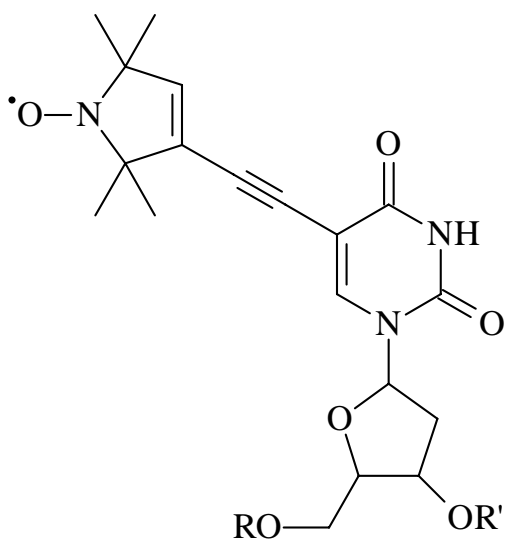

b

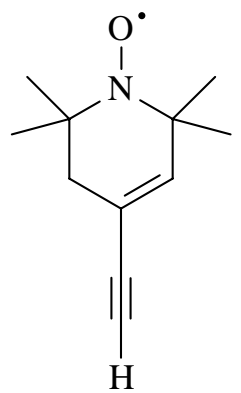

c

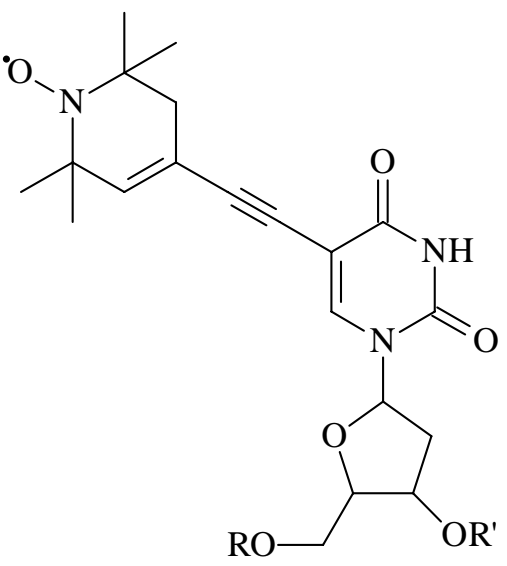

$\mathrm{d}$

Figure 1.5. Chemical structures of a) 5 -membered ring nitroxide with acetylenic tether (5sp), b) $5 \mathrm{sp}$ phosphoramidite analogue, c) 6-membered ring nitroxide with acetylenic tether (6sp), b) 6sp phosphoramidite analogue.

However, the difficulty of synthesizing ${ }^{\mathbf{5} p \mathbf{T}}$ and particularly the spin label $\mathbf{5 s p}$, motivated us to come up with more synthetically accessible and more easily prepared probes to facilitate their use. Therefore, we designed and synthesized a new spin label, 6sp (Figure $1.5 \mathrm{c}$ ) and thus continued to the synthesis of phosphoramidite ${ }^{6 \mathbf{s p}} \mathbf{T}$ (Figure $1.5 \mathrm{~d}$ ). The later phosphoramidite was prepared in 6 steps from readily available starting materials compared to the former, which required 12 steps $^{22}$. In addition, we have shown that these two spin labels 
are equivalent based on the EPR, CD, UV, and thermal denaturation spectra of single, double and triple stranded oligonucleotides.

The interplay between experimental and theoretical approaches greatly enhances our knowledge in understanding and interpreting the results. In order to better understand biological information transfer, molecular interactions of nucleic acids and polymorphic character of nucleic acid conformation, it is important to understand the structure, dynamics, stability and relative flexibility of ds DNAs and tx DNAs as well as DNA-RNA duplexes. A large number of theoretical calculations of nucleic acid structure have been performed ${ }^{23} 24$. Combination of molecular mechanics (MM) and statistical mechanics, computational chemistry of nucleic acids has made impressive breakthroughs beyond the pure quantum chemical studies ${ }^{25,26,27}$. Feasibility of oligonucleotides with modified bases has been studied with molecular modeling ${ }^{28}$. Simple modeling was used as a guide in the initial design of stable backbone modification to DNA proposed for use as an antisense therapeutic agent ${ }^{29}$. The application of computer-based models using analytical potential energy functions within the framework of classical mechanics has proven to be an increasingly powerful tool for studying structure and dynamics of small organic molecules or larger, biologically interesting, molecules. These methods have lead to an increased understanding of DNA structure, conformation and stability. Likewise they can be used to suggest or guide new research. In this work we will discuss some aspects of molecular dynamics simulations performed on several DNA molecules with and without modified nitroxide spin-labeled bases, comparing their stability and relative motion of the nitroxide with respect to the DNA molecule.

Molecular Dynamics (MD) simulations provide a useful and inexpensive method for investigating structure, stability and conformations of DNA structures in solution. Recently, 
MD simulations were adopted to study the conformational properties of short helixes including water molecules and ions, the effect of 8-oxodeoxyguanosine substitution on the stability of double stranded DNA ${ }^{30}$. DNA duplexes with G-T mismatces and methylphosphonate substituted backbone were also studied with MD simulations. In the present work we are extending the use of $\mathbf{5 s p}$ and $\mathbf{6 s p}$ modified DNAs in application to MD simulations to investigate the effect of these modified bases ${ }^{5 \mathrm{sp}} \mathbf{T}$ and ${ }^{6 \mathrm{sp}} \mathbf{T}$ (Figure 1.5). The stability, conformation and dynamics aspects of single stranded (ss), double stranded (ds) and triple stranded or triplex (tx) DNAs are considered as a result of spin label incorporation. Also, the relative motion of the nitroxide with respect to the DNA molecule is considered.

Suitable parameters for NO moiety of nitroxide type atoms using two training nitroxides MNO and TBNO have developed. In addition a new type of carbon atom C1, involved in acetylenic tether of the nitroxide, (for $s p$-hybridized carbon) with corresponding parameter was also developed and added to the Amber parm94 force field. MD simulations on both unmodified and 5 sp and 6 sp modified 15-mer ss, ds, and tx DNA models were conducted by inserting the spin-labeled analogue of thymidine ${ }^{5 \mathrm{sp}} \mathbf{T}$ and ${ }^{6 \mathrm{sp}} \mathbf{T}$ into the middle part of $d\left(\mathrm{~T}_{7} \mathrm{~T}^{*} \mathrm{~T}_{7}\right)$ strand.

Incorporation of the modified thymidines ${ }^{5 \mathrm{sp}} \mathbf{T}$ and ${ }^{6 \mathrm{sp}} \mathbf{T}$ does not lead to any significant increase in steric interactions, since these probes are directed away from and perpendicular to the DNA helical axis. The changes in helical parameters of the duplex to accommodate the spin label in the major groove are observed. There is some experimental evidence, that the acetylene tether of the nitroxide actually improves the stability of tx DNA formation. With the aid of molecular dynamics simulation the source of this improved stability has been explored. Global structural parameters of modified and unmodified triplexes are also 
compared. In contrast to duplexes, we did not see any noticeable changes in triplex structure of spin labeled DNA compared to unmodified one.

Single stranded oligonucleotides are very flexible compared to duplexes, because they do not have a base paring complementary strand to keep the molecule rigid. So, the simulations of ss DNAs become very tricky. By introducing the modification, drastic conformational changes were observed when unconstrained MD was employed. Information about the rate of the motion of the nitroxide calculated from the MD trajectories can be related to the correlation time $\left(\tau_{\mathrm{c}}\right)$. In order to calculate correlation times from MD trajectories, the theoretical model based on the statistical random walk was used.

Description of experimental techniques used in the current work is outlined in chapter 2. The experimental details of the synthesis of spin labeled oligonucleotides and sample preparation for spectroscopic measurements along with results and discussion are presented in chapter 3. Theoretical background of methods used in parameter development and simulations of DNAs is given in the following chapter 4. Chapter 5 describes computational methods and Molecular Dynamics simulation results of single, double and triple stranded unmodified and spin labeled DNAs. 


\section{CHAPTER 2}

\section{Experimental Techniques}

Several experimental techniques have been used in the present work. They include thermal denaturation studies, circular dichroism (CD), electron paramagnetic resonance (EPR), and nuclear magnetic resonance (NMR) spectroscopies. Combination of all these techniques is important for establishing the relevance of the conditions used in either of these techniques to achieve high quality results, for examining the conformational changes or complex formation, for example of DNA with oligonucleotides. In addition these processes can be monitored at a real time scale with, for example EPR technique.

In most cases, oligonucleotide complexes are stable at room temperature, which makes it difficult to determine the relative strength of different base paired interactions. By changing one of the most commonly used environmental variables, temperature, one could quantitatively study these complexes. If we monitor the absorbance of the homopolymer as a function of temperature, we can notice a sharp increase centered at some characteristic value. This is called the melting temperature, $T_{m}$, and it is specific for each sample. The sharpness of the transition approaches that of true phase transition between two phases of the molecule, such as double stranded and single stranded phases or alternatively melting of a triplex to a double and single strands (Figure 2.1.).

Although above and below the melting region the temperature could be quite constant, the properties of single strands can change with temperature. For example, some duplexes that melt at lower temperatures will produce partially stacked single strands. As the temperature is raised, they will show additional changes in absorbance. 


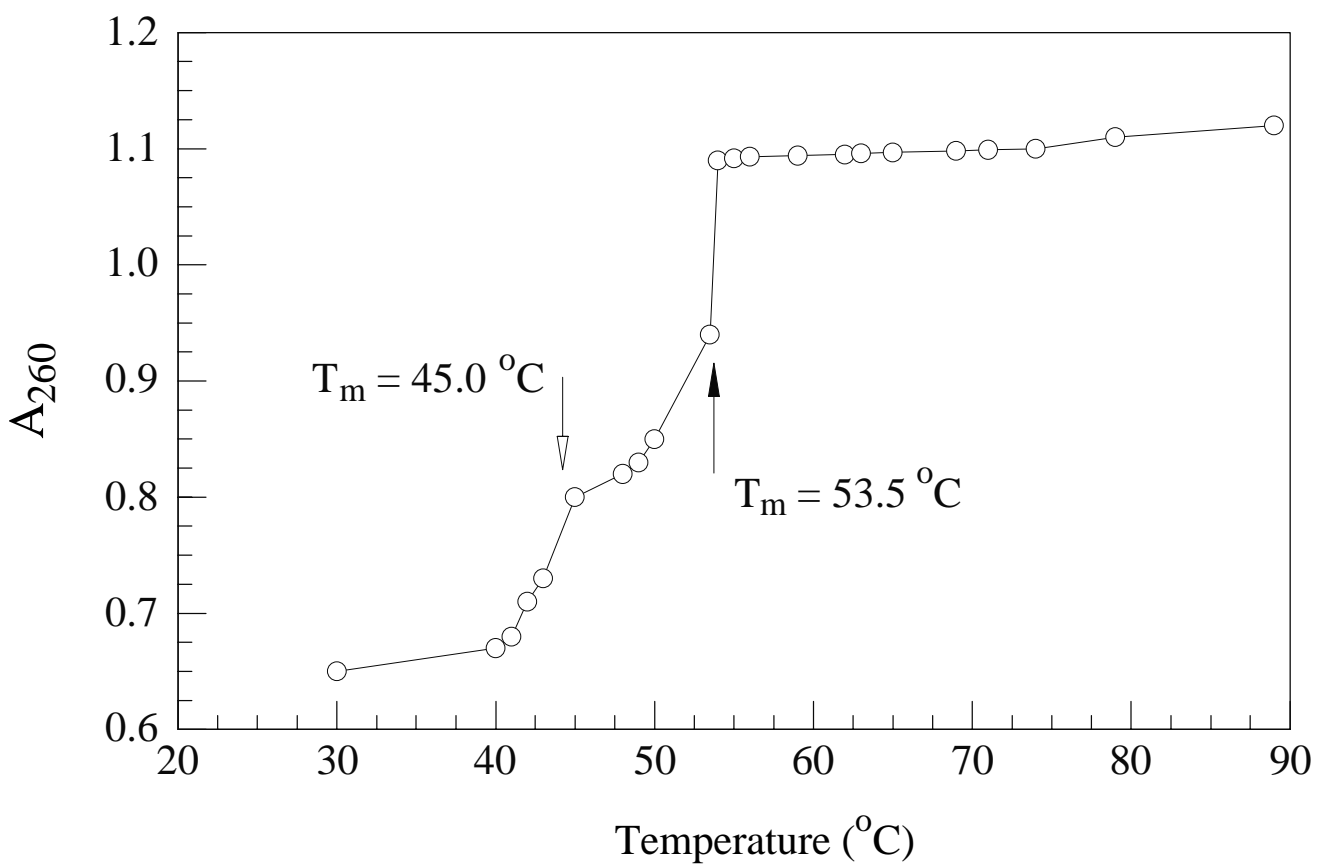

Figure 2.1. Two-step melting of U:A-U corresponding to formation of polyA;polyU and polyU at $45 \mathrm{C}$ and all single strands at 53..5 C. [After C. Stevens and G. Felsenfeld, Biopolymers 2:293 (1964).]

Circular dichroism (CD) spectroscopy ${ }^{31,32}$ is a form of light absorption spectroscopy that measures the difference in absorbance of right- and left-circularly polarized light (rather than the commonly used absorbance of isotropic light) by a substance. The phenomenon of circular dichroism is very sensitive to the secondary structure of biological molecules. The analysis of CD spectra between 300 and approximately $180 \mathrm{~nm}$ can therefore yield valuable information about different secondary structures of nucleic acids, polypeptides such as duplex and triplex DNAs, alpha helix, parallel and antiparallel beta sheet, turn, and other molecules.

EPR and NMR ${ }^{33-35}$ are powerful analytical tools widely used in many disciplines, such as physics, chemistry, biochemistry, material sciences, etc. to study a variety of systems. EPR requires microwave frequency, while NMR usually requires radiofrequency to induce 
the splitting of energy levels by a magnetic field. This splitting is referred to as the Zeeman effect. So, one can say that EPR and NMR study the energy required to reorient electronic and nuclear magnetic moments of the system in question, in magnetic field. The main feature of and a necessary requirement for an EPR experiment is that it is applicable to systems with a net electron spin angular momentum, i.e. systems with one or more unpaired electrons. A respectable number of systems fulfill this condition. These are (1) free radicals in the solid, liquid, or gaseous states, (2) biradicals, which can be considered as two slightly interacting

free radicals, (3) spin labels, (4) systems with two, three, or more radicals, (5) point defects in inorganic crystals (such as transition-metal ions and rare-earth ions). In this chapter the basic principles of Tm, CD, EPR, and NMR with discussion in application to nucleic acids are described.

\subsection{Circular Dichroism Spectroscopy (CD)}

Circularly polarized light plays an important and decisive role in CD spectroscopy. When a molecule is illuminated with a linearly polarized light it induces oscillating electric and magnetic fields in the molecule. These fields in turn invoke the oscillation of electrons and nuclei in the molecule. Electrons, being lighter than nuclei, will be polarized along the direction of the oscillating electric field vector $\mathbf{E}$.

In order to observe optical activity in a solution, two conditions must be satisfied: first, a substance must possess neither a center nor a plane of symmetry, and second, either plane or circularly polarized light must be employed in studying the interaction of electromagnetic radiation with matter. 
When the light passes through the optically active medium, it suffers no change in polarization. However, it will be partially absorbed, and the degree of absorption will depend upon whether the light is left or right circularly polarized. Since there is no known dichroic material that possesses extinction coefficients of different strengths in a broad spectral region, the circularly polarized light is produced with a polarizer, which is followed by a birefringent plane that has a slow and a rapid axes. Two in-phase vibrations parallel to the axes pass through the plate with different velocities. Hence, passing through the medium the speeds of right and left circularly polarized light change, i.e. $c_{L} \neq c_{R}$. This in turn leads to the wavelengths of these rays being different, $\lambda_{\mathrm{L}} \neq \lambda_{\mathrm{R}}$, and also these two rays are absorbed to a different extent, which produces different extinction coefficients $\varepsilon_{L} \neq \varepsilon_{R}$. This difference in absorbance $\Delta \varepsilon \equiv \varepsilon_{\mathrm{L}^{-}} \varepsilon_{\mathrm{R}}$ is called circular dichroism. Ellipticity is another effect caused by circular dichroism. Putting in quantitative terms, one can describe circular dichroism, in terms of Beer-Lambert-Bouguer law, which states that the absorbance A is proportional to the concentration $c$ and pathlength $l$ of a sample

$$
A=\log _{10} \frac{I_{0}}{I}=\varepsilon c l
$$

where $\varepsilon$ is molar extinction coefficient, $\mathrm{I}_{0}$ and $\mathrm{I}$ are the intensities of incident and leaving light, respectively. In an optically active medium, which is the main requirement for $\mathrm{CD}$, one can record two such absorptions for left $A_{L}$ and right $A_{R}$ circularly polarized light with respective leaving intensities $I_{L}$ and $I_{R}$ (note that $I_{0 L}=I_{0 R}$ ). Then the equation 2.1 can be rewritten as

$$
\Delta A=A_{L}-A_{R}=\log _{10} \frac{I_{0}}{I_{L}}-\log _{10} \frac{I_{0}}{I_{L}}=\log _{10} \frac{I_{R}}{I_{L}} \text {, hence }
$$




$$
\Delta \varepsilon=\frac{1}{c l} \Delta \mathrm{A}
$$

Figure 2.2 depicts both a plane-polarized (Figure 2.2a) light produced by combination of coherent right and left circularly polarized lights of equal intensities, and elliptically polarized (Figure 2.2b) light.

a)

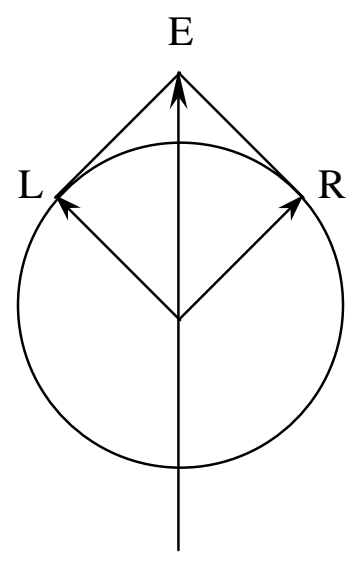

b)

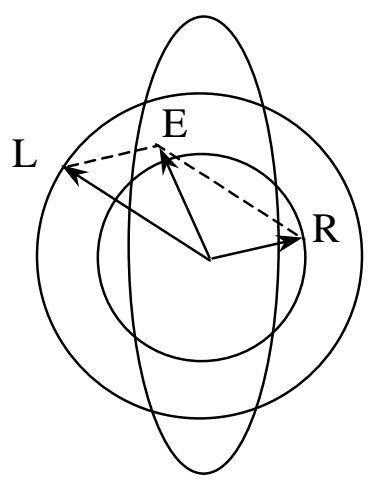

c)

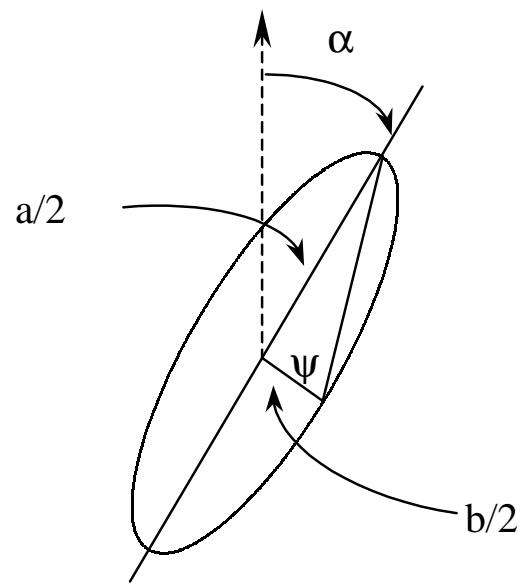

Figure 2.2. a) Plane circularly polarized light resolved into its left $L$ and right $R$ components of similar intensities. b) Different absorbance of right (more absorbed) and left (less absorbed) components of the light result in elliptically polarized light with corresponding ellipse for the path of electric vector $\mathbf{E}$. c)

Nucleic acids are complex optically active molecules and hence their properties are constructed from individual groups they are comprised of. The chromophores that make up nucleic acids are phosphate-sugar backbone and an unsaturated benzene-like base. The electronic transitions of phosphates occur only at high energy, at wavelengths shorter than $170 \mathrm{~nm}$, while sugars have low-intensity bands starting at $\sim 190 \mathrm{~nm}$. The sugar transitions are mostly involve $\sigma \sigma^{*}$ transitions and their contribution to $\mathrm{CD}$ are generally weak. Thus, in a convenient wavelength ranges $(180-700 \mathrm{~nm})$ the bases are the important ones in 
spectroscopic terms, since they have strong electronic transitions in the near- and far-UV region. All the heavy atoms have $2 p$ orbitals that overlap to form a benzene-like $\pi$ molecular orbitals. Each of these orbital contain $\pi$ electrons and only the lowest-energy $\pi$ orbitals will be filled making possible a large number of $\pi-\pi^{*}$ transitions to occur from low-energy filled $\pi$ orbitals to higher-energy unfilled $\pi$ orbitals. Though the bases do not have intrinsic optical activity, because they are symmetric and have a plane of symmetry, but since they are attached to the sugars, which in turn are asymmetric groups, a CD in the absorption bands of chromophoric bases can be induced. These bands can be observed either for the intense electronically allowed $\pi-\pi^{*}$ transitions, or for the weaker $n-\pi^{*}$ transitions. The later ones are possible because they are magnetically allowed.

The secondary structure of nucleic acids is polymorphic, meaning that the number of base pairs per turn, inclination of base pairs with respect to the helical axis, the handedness, base composition and other parameters are all solvent dependent. The CD spectroscopy is exquisitely sensitive to nucleic acid secondary structure, since it depends on base-base interaction and stacking. It is the method of choice for following changes in secondary structure, conformation and other properties as a function of solvent conditions.

When the DNA is in aqueous solution with moderate amount of sodium salt, it is presumed to be in the, historically termed B-form, the right-handed conformation. In this conformation B-DNA has a characteristic positive band centered at $275 \mathrm{~nm}$ wavelength. With changes in salt concentration one can monitor the collapse of this band, while the reminder of the spectrum remains unchanged. This is how P-form DNA was discovered, while investigating the effect of ethanol and methanol mixtures on the A- and B-forms of DNA. Depending on the base composition and solvent condition the B-form, for example poly-GC 
sequence, can be converted to Z, left handed DNA form. With the help of CD spectroscopy the first Z-form DNA was observed and also shown, that while in the B-form that sequence is quite different from the poly-(AT) one. The differences are observed in the intensities at long wavelength positive and a negative band at $210 \mathrm{~nm}$. This once again emphasizes that the CD spectrum is sequence dependent.

The application of CD spectroscopy has been extended to non-classical new types of structures such as triplexes, quadruplexes, and modified oligonucleotides. In particular, CD spectra are useful for the determination of complex formation between modified oligonucleotides and its target. Several parameters, for instance, the stoichiometry of complex formation or the melting temperature of hybrid can be determined. Hairpin formation within a single oligonucleotide with dangling arm can be observed with $\mathrm{CD}$. By adjusting the $\mathrm{Mg} 2+$ salt concentration one can achieve formation of two types of triple helices, parallel and antiparallel. The differences between these two types can be detected by this technique. One can easily follow thermal denaturation of these types of structures allowing therefore the comparison of the two triplexes. All these points make CD powerful technique in application to our studies.

\subsection{Principles of Electron Paramagnetic Resonance (EPR)}

Since its discovery about half a century ago by Zavoiskii the EPR technique has provided detailed structural information on a variety of organic and inorganic systems of paramagnetic nature. It was a great continuation of one of the early experiments of Stern and Gerlach when they showed that an atom with an electron magnetic moment could only have 
discrete orientations a magnetic field. This brings to the notion that electrons and nuclei obey quantum rather than classical mechanics. As a quantum-mechanical particle, it has an associated deBroglie wavelength $\lambda=h / p$, where $h=6.6260755 \cdot 10^{-34} \mathrm{~J} \mathrm{~s}$ is Planck's constant and $p$ is linear momentum, when considering the motion in a ring. The square of amplitude of the $\lambda$ gives the probability of finding this particle at any point on the ring. The wave function should be single-valued in order for the probability to be time-independent, otherwise the wave will destroy itself in propagating about the ring. Then we can write

$$
2 \pi r=\mathrm{m} \lambda=\mathrm{m} h / p
$$

where $\mathrm{m}=0,1,2,3, \ldots$, etc. and hence

$$
p r=p_{\phi}=\mathrm{m} h / 2 \pi \equiv \mathrm{m} \hbar
$$

For $\mathrm{m}=1$ the electron is said to be in a $p$ orbital, which is the case for most stable free radicals. This simple model leads to the fundamental phenomenon of quantization of angular momentum of an electron. In fact, the total orbital angular momentum should be considered, which includes both orbital angular momentum and an intrinsic magnetic moment, which is called spin of an electron. These two momenta are coupled to give rise to so-called spin-orbit interaction. This type of interaction is usually small for free radicals and hence could be ignored.

The basic idea behind an EPR experiment is that it studies the interaction between electronic magnetic moments and magnetic field. Magnetic dipole transitions are induced between energy levels of electron spins when they are placed in a static magnetic field $B$. The energy of the magnetic dipole in a magnetic field then can be expressed as

$$
E=-\vec{\mu} \cdot \vec{B}
$$


where $\mu$ is the dipole and $\mathrm{B}$ is the magnetic field vector. By considering $\mathrm{B}$ along the $\mathrm{z}$-axis, the component of electron spin magnetic moment can be written as

$$
\mu_{\mathrm{Z}}=-\mathrm{g} \beta \mathrm{m}_{\mathrm{S}}
$$

Here, $g=2.00232$ for a free electron, $\beta$ is the Bohr magneton, and $m_{S}$ is the projection of the spin on the z-axis. The minus sign is accounted for the negative charge of the electron. Substitution of equation (2.3) into equation (2.4) yields the following expression for the energies

$$
E=g \beta B m_{S}
$$

The allowed values of the spin quantum number $\mathrm{m}_{\mathrm{S}}$ range from $-\mathrm{S}$ up to $+\mathrm{S}$, with unit increments, giving total of $2 S+1$ components. For the system with the spin $S=1 / 2$, the allowed values for $m_{S}$ are $+1 / 2$ for "spin up" state $(\uparrow)$ and $-1 / 2$ for "spin down" state $(\downarrow)$. These are referred to as the Zeeman energy levels. The separation $\mathrm{g} \beta \mathrm{B}$ between the Zeeman levels linearly depends on the magnitude of magnetic field. This dependence is illustrated in Figure 2.3.

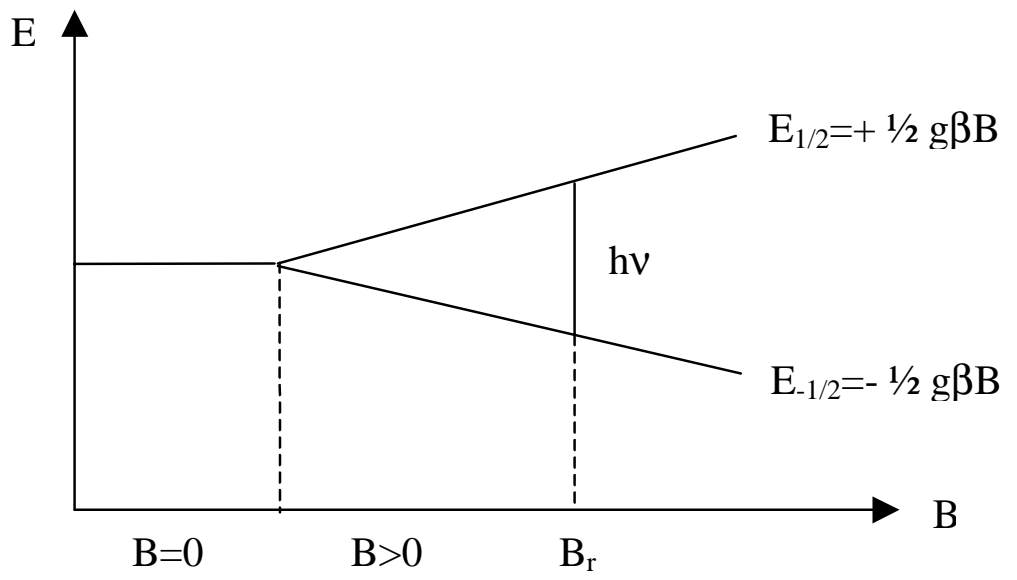

Figure 2.3. Energy level scheme as a function of the applied magnetic field $B . E_{1 / 2}$ and $E_{-1 / 2}$ represent the energies of $m_{S}=1 / 2$ and $m_{S}=-1 / 2$ states, respectively. $B_{r}$ is a resonant magnetic field. 
In order to induce a transition between lower state to an upper excited state an electromagnetic radiation of appropriate frequency $v$ must be applied. Whenever the energy of the photon hv matches the energy separation between the two states, resonance occurs. Then

$$
\Delta \mathrm{E} \equiv \mathrm{E}_{2}-\mathrm{E}_{1}=\mathrm{h} v=\mathrm{g} \beta \mathrm{B}_{\mathrm{r}}
$$

where $v$ is a frequency of applied field and $B_{r}$ is a static magnetic field at which the resonance condition is fulfilled. The necessary condition required by the so-called selection rule $\Delta \mathrm{m}_{\mathrm{S}}=$ \pm 1 must be satisfied in order to have an allowed EPR transition.

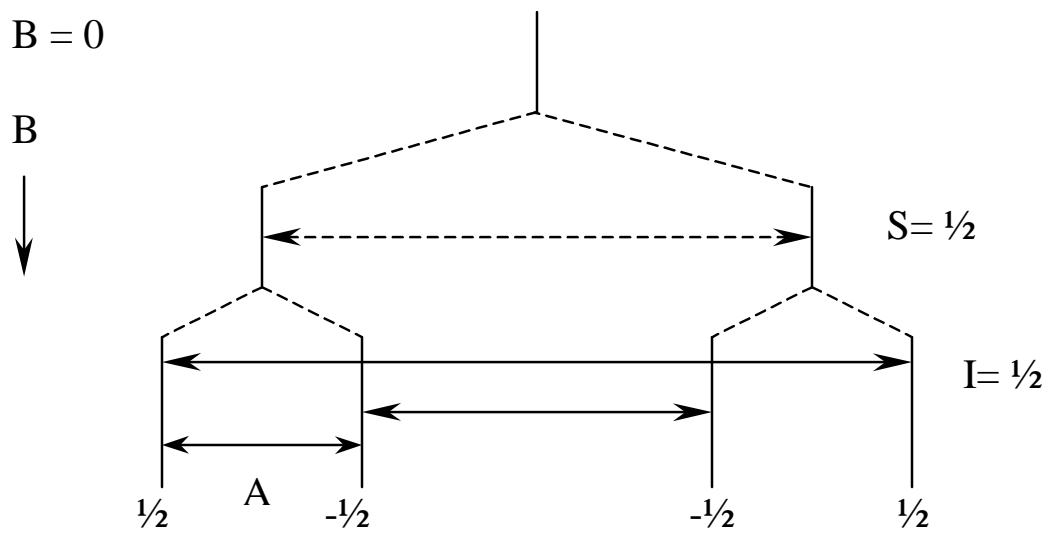

Figure 2.4. Energy levels of hydrogen atom, showing hyperfine splitting. Solid lines indicate allowed transitions. For $\mathrm{A}=0$ transition is indicated with dotted line.

Usually the interaction of an electron with the magnetic field is not the only effect responsible for EPR spectra. Often times there are other interactions that can produce spectra with multiple lines and in fact, it is these interactions that render the technique useful. The interaction of an unpaired electron with one or more nuclei in its vicinity, which posses nuclear magnetic moments, is called nuclear hyperfine interaction. The hyperfine interaction 
causes additional splitting of Zeeman energy levels. As a simple example, consider the interaction of $\operatorname{spin} S=1 / 2$ with nucleus of $I=1 / 2$. The diagram of energy levels of hyperfine splitting of hydrogen atom is shown in Figure 2.4.

Like in the case of an electron, the nuclear energy levels are also quantized and they are characterized by the nuclear quantum number, $\mathrm{m}_{\mathrm{I}}$. Since the proton has a sipn $\mathrm{I}=1 / 2$, the two allowed values for $m_{I}$ are $\pm 1 / 2$, the number of energy levels is given by $(2 S+1) \times(2 I+1)$, and in order to have allowed transitions, the following selection rule must be met:

$$
\Delta \mathrm{m}_{\mathrm{S}}= \pm 1, \Delta \mathrm{m}_{\mathrm{I}}=0
$$

The transitions will occur at two resonant fields $B_{\mathrm{r} 1}$ and $\mathrm{B}_{\mathrm{r} 2}$. The resulting EPR spectrum of these transitions is shown in Figure 2.5. Here the separation between lines is constant and A is denoted as the hyperfine coupling constant.

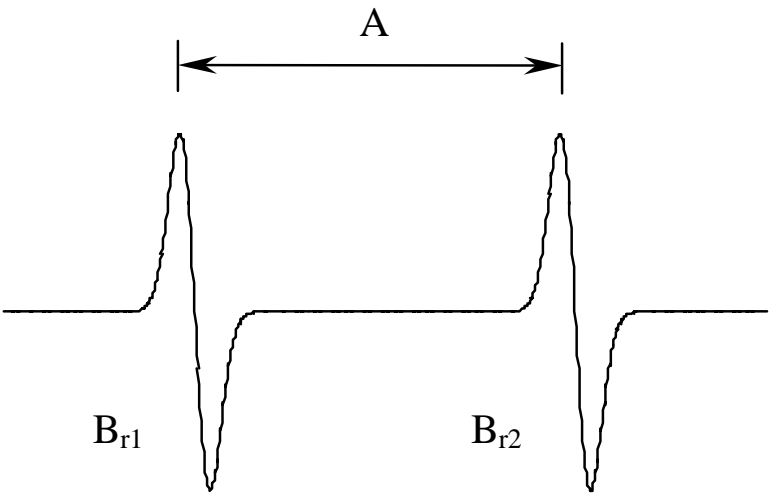

Figure 2.5. EPR spectra reflecting resonant field conditions $B_{r 1}$ and $B_{r 2}$. A is hyperfine coupling constant.

Usually, for many paramagnetic systems, both $g$ and A values are isotropic. Nitroxides and other stable free radicals produce spectra whose linewidth and lineshape can reflect the anisotropic environment of the molecule they are attached to (spin labels) or occupy such 
positions near molecules (spin probes). The most commonly used spin labels produce a threeline hyperfine pattern due to the interaction of an unpaired electron of the nitrogen atom with its nucleus $(\mathrm{I}=1)$. In general both $\mathrm{g}$ and A values are anisotropic with the following principal values:

$$
\begin{array}{ll}
\mathrm{g}_{\mathrm{x}}=2.008 & \mathrm{~A}_{\mathrm{x}}=5.5 \mathrm{G} \\
\mathrm{g}_{\mathrm{y}}=2.006 & \mathrm{~A}_{\mathrm{y}}=4.0 \mathrm{G} \\
\mathrm{g}_{\mathrm{z}}=2.003 & \mathrm{~A}_{\mathrm{z}}=30.0 \mathrm{G}
\end{array}
$$

These values are determined from the powder or frozen solution samples of the nitroxide at low temperatures well below $0 \mathrm{C}$. When going room temperature to the frozen sample, the spectral line change dramatically (Figure 2.6). In the common case, when the spin label is not completely immobilized the distance between the outer wide extremes of EPR spectrum is $2 \bar{A}_{\|}$. For the case of a frozen spectrum (Figure 2.6a) this value is equal to $2 \mathrm{~A}_{\mathrm{z}}$, where $\mathrm{A}_{\mathrm{z}}$ is a maximal principle value of the hyperfine tensor $\mathbf{A}$. To describe molecular motion of the spin label one can use the order parameter S, which is calculated from the following expression

$$
S=\frac{\bar{A}_{\|}-\bar{A}_{\perp}}{A_{Z Z}-1 / 2\left(A_{X X}+A_{Y Y}\right)}=\frac{\bar{A}_{\|}-A_{0}}{A_{Z Z}-A_{0}}
$$

where the $A_{0}$ is the isotropic hyperfine constant and the expression for $A_{0}$ was used

$$
A_{0}=\frac{1}{3}\left(\bar{A}_{\|}+2 \bar{A}_{\perp}\right)=\frac{1}{3}\left(A_{X X}+A_{Y Y}+A_{Z Z}\right)
$$


a)

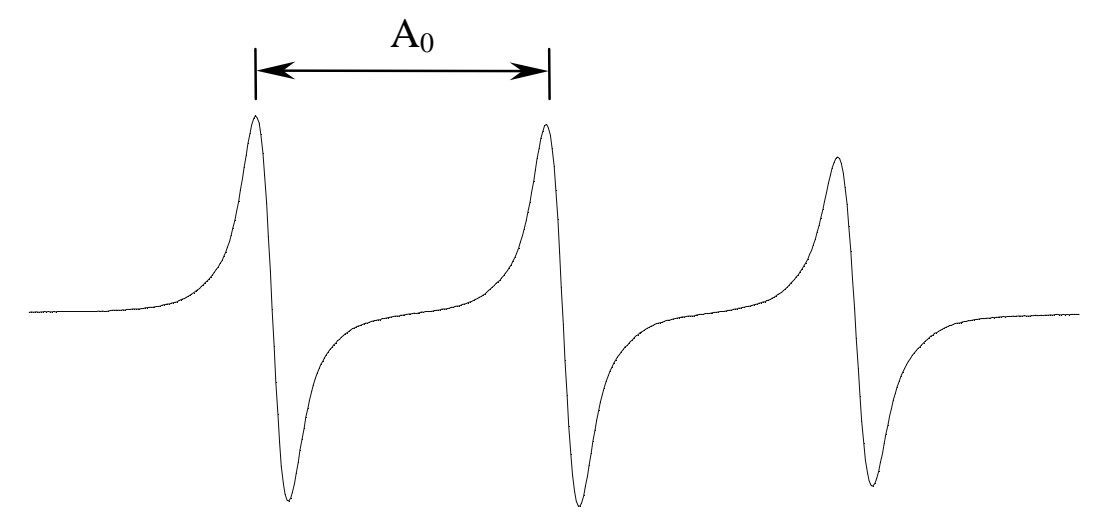

$\mathrm{A}_{\mathrm{z}}$

b)

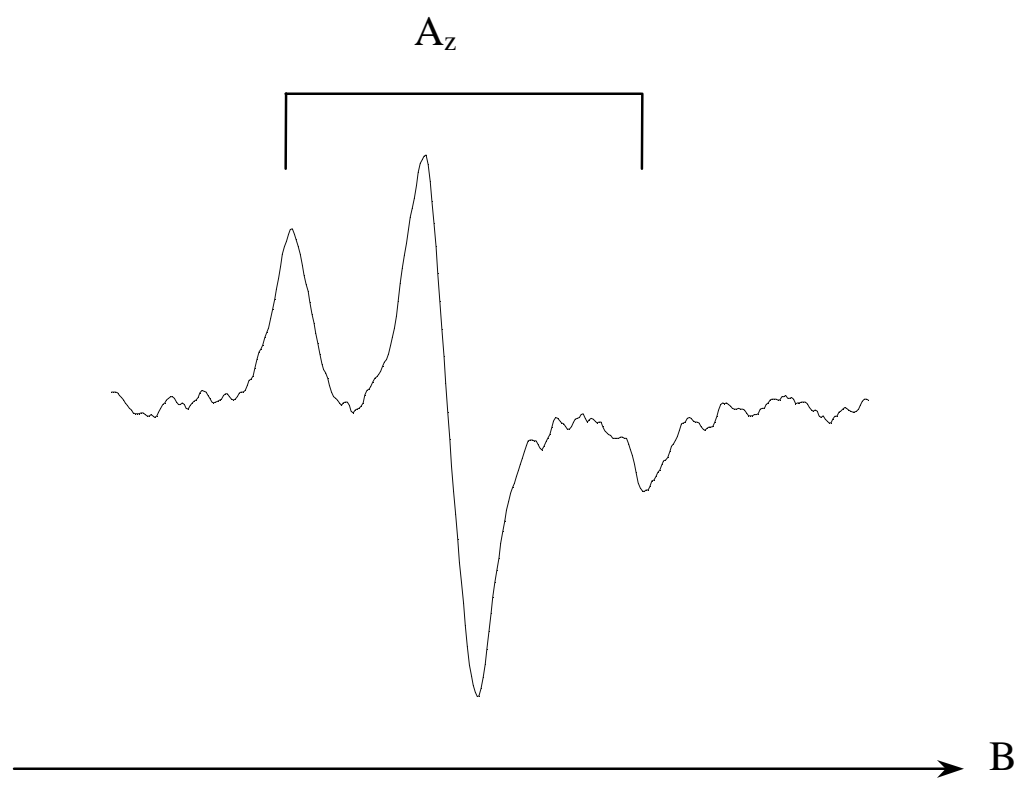

Figure 2.6. EPR spectra of nitroxide molecule at room temperature (a) and frozen sample (b).

\subsection{Nuclear Magnetic Resonance (NMR)}

Nuclear magnetic resonance (NMR) is another type of absorption spectroscopy such as EPR or UV spectroscopy. In contrast to EPR, where microwave frequency is used to 
induce transitions in a sample placed in the magnetic field, in NMR one needs a radio frequency $(r f)$ to achieve the resonance condition. When placed in the static magnetic field $\mathbf{B}_{0}$, the sample will absorb electromagnetic radiation, $\mathbf{B}_{1}$, in the $r f$ region at frequencies governed by the characteristics of the sample. Absorption will depend on the identity of the nuclei in the molecule. Under the influence of $\mathbf{B}_{0}$ the nuclear spins are polarized, resulting in the net macroscopic magnetization $\mathbf{M}$. The direction of magnetization is coincident with the magnetic field $\mathbf{B}_{0}$. Figure 2.7 illustrates a simple, classical picture of magnetization as a result of applied magnetic

a)

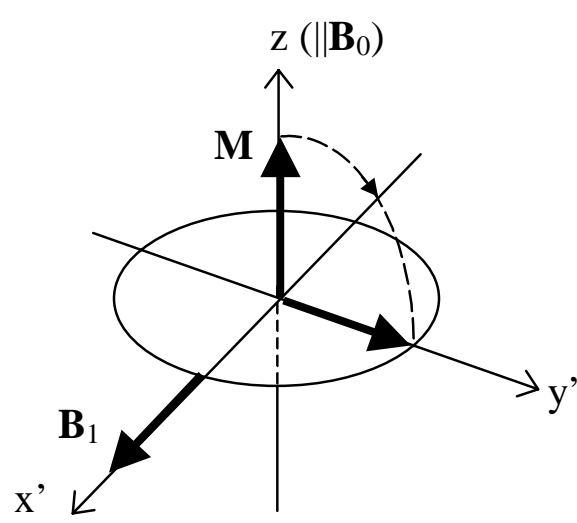

b)

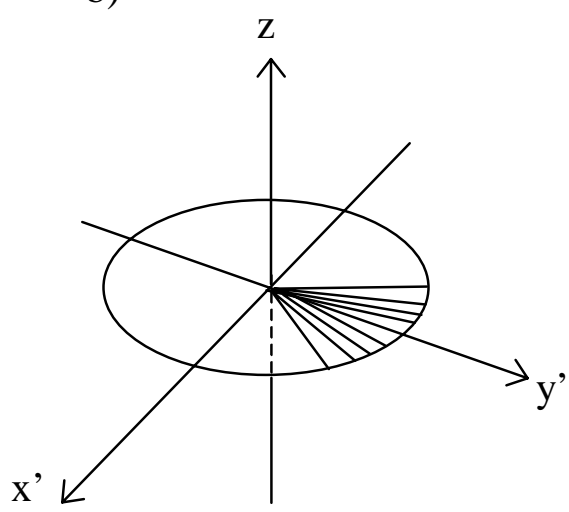

Figure 2.7. (a) Effects of magnetic fields on a NMR sample. A rotating frame coordinate system is defined by $x^{\prime}, y^{\prime}$, and $z$, with $z$ along $\mathbf{B}_{0}$. (b) Decay of phase coherence during precession of the magnetization in the $x-y$ plane.

field. In order to flip magnetization towards the $x-y$ plane perpendicular to $\mathbf{B}_{0}$, the $r f 90^{\circ}$ pulse of appropriate duration is applied, which is accomplished by a coil with its axis perpendicular to $\mathbf{B}_{0}$. The transverse magnetization in the $x-y$ plane precesses under the influence of the static magnetic field at the resonant frequency $v_{0}$. This frequency induces an electric current in the detection coil, which is a primary observation in NMR experiment. The 
fundamental NMR equation correlating electromagnetic frequency with magnetic field strength is

$$
\omega_{0}=\gamma \mathbf{B}_{0}
$$

where $\omega_{0}=2 \pi v_{0}$ is the Larmor frequency, and $\gamma$ is called magnetogyric ratio and is a fundamental nuclear constant. A frequency of 100 megahertz $(\mathrm{MHz})$ is needed at a magnetic field $\mathbf{B}_{0}$ of 2.33 Tesla (T) for proton. For the purposes of detecting biopolymers with NMR a magnetic field of $11.74 \mathrm{~T}$ or greater is typically used. The corresponding frequency for proton is $500 \mathrm{MHz}$. When the system is returning to its thermodynamic equilibrium, which is the case of $\mathrm{M} \| \mathrm{B} 0$, the transverse magnetization decays over time in a manner designated as free induction decay (FID). The NMR spectrum represents a Fourier Transform (FT) of the FID data recorded during a time of approximately 1 second.

The characteristic of the resonant frequency of a nucleus depends to a very, but measurable (several parts per million (ppm)), extent upon its chemical environment. This allows the NMR spectrum to have not a single resonant value of $B_{0}$ at particular rf $v_{0}$, but multiple resonant values. The total range of variation of $B_{0}$ for protons is typically about 13 ppm or $2600 \mathrm{~Hz}$ in a $4.73 \mathrm{~T}$ field. The origin of this variation in resonant field strength is the cloud of electrons that are surrounding the nucleus. Electrons under the influence of magnetic field will generate their own small, local magnetic fields, which will be proportional but opposite in direction to $B_{0}$, hence the shielding effect. The degree of shielding depends on the density of circulating electrons and on the electronegativity of nearby groups or atoms. The difference in the absorption position of a particular proton from some internal reference proton is called chemical shift $(\delta)$ of the particular proton. The most commonly used reference is tetramethylsilane (TMS), because of its single sharp absorption peak at a higher 
field than almost all organic protons. While NMR became a widely used technique in structure determination of relatively small molecules, its application to macromolecules is still a matter of investigation.

Corresponding to the large size of the molecules, the NMR spectra of biopolymers are densely crowded with resonance lines. Nowadays, determination of protein structure by NMR methods is widely accepted by both NMR spectroscopists and crystallographers. Application of these methods to nucleic acids has slowed owing primarily to the lower proton density of nucleic acids relative to proteins. But, with the advent of convenient and rapid DNA synthesis methods and two-dimensional (2D and 3D) NMR, a wealth of details on the molecular conformations have become accessible for experimental observations with NMR. Among the possible conformations investigated by NMR are single stands (ss), stacked helical ss, hairpins, regular duplexes, etc. Most resonance lines have different chemical shifts in the single strand and in the duplex. The lines in the duplex become more broadened, owing to the comparative rigidity of the structure, with limited segmental mobility for the individual strands. Thus, NMR studies of more complex conformations, such as triple stranded DNAs, become even more difficult. Special conditions must be employed in order to obtain readable spectra. Also, for gathering high-resolution data, NMR spectrometer operating at frequencies higher than $500 \mathrm{MHz}$ will help in advancing in structure determination of unusual nucleic conformations. 


\section{CHAPTER 3}

\section{Experimental Procedures or Details}

A variety of DNA structures such as single and double stranded DNAs, loops that can be formed in both stem and helical regions, and base pair mis-matches and bulges have been studied with spin labels. In particular, spin labels based on 5-membered ring nitroxide (5sp) have been widely used. The potential of triplex DNAs to affect gene expression and have therapeutic significance brought up the idea of using these spin labels to monitor triplex formation. To our knowledge, this spin labeling technique has not been used to examine triplex DNA (tx DNA) formation. In our lab, Jeannine Powell first started the synthesis of 5sp nitroxide. However it was difficult to synthesize this spin label and it requirs twelve steps. Similar, more synthetically accessible probes, would facilitate their use. This idea motivated us to design and synthesize a new spin label, based on 6-membered ring nitroxide (6sp) and its phosphoramidite analogue, which can be prepared in 6 steps from readily available starting materials. In this study we show that this new spin label can also be used for detecting and monitoring triplex DNA formation.

Solvents and reagents were obtained from Aldrich (Milw, WI) and were used without purification unless otherwise noted. Unmodified phosphoramidite DNA bases and CPG resins were obtained from Glen Research (Sterling, VA). Unless otherwise stated, solutions of samples for Tm, CD, and EPR were prepared in phosphate buffer $\left(10 \mathrm{mM}, \mathrm{NaH}_{2} \mathrm{PO}_{4}, \mathrm{pH}\right.$ 7.4), and $100 \mathrm{mM} \mathrm{NaCl}$. Magnesium ion concentrations of 10,25 or $50 \mathrm{mM}$ were achieved by the addition of aliquots of $1.75 \mathrm{M} \mathrm{MgCl}_{2}$ to the solution of the duplex or triplex. 


\subsection{Synthesis}

\subsubsection{Phosphoramidite synthesis}

4-Ethynyltriisopropylsilyl-4-hydroxy-2,2,6,6-tetramethyl-piperidine-1-oxyl (2): $\quad$ A solution of $\mathrm{n}-\mathrm{BuLi}(11.66 \mathrm{mmol})$ was added slowly to a solution of triisopropylsilylacetylene $(2.025 \mathrm{~g}, 11.1 \mathrm{mmol})$ and anhydrous $\operatorname{LiBr}(1.836 \mathrm{~g}, 21.14 \mathrm{mmol})$ in dry THF $(30 \mathrm{~mL})$ under $\mathrm{N}_{2}$ at $0{ }^{\circ} \mathrm{C}^{36}$. The mixture was stirred for $30 \mathrm{~min}$ and then a solution of 4-oxo-2,2,6,6tetramethyl-1-piperidinyloxy (1) $(1.8 \mathrm{~g}, 10.57 \mathrm{mmol})$ dissolved in dry THF $(30 \mathrm{~mL})$, was added over 1 hour via a syringe pump, and the solution stirred overnight at $20^{\circ} \mathrm{C}$. The reaction was quenched by the addition of saturated aqueous $\mathrm{NH}_{4} \mathrm{Cl}(15 \mathrm{~mL})$, extracted with $\mathrm{CH}_{2} \mathrm{Cl}_{2}(4 \times 40 \mathrm{~mL})$, the organic phase dried $\left(\mathrm{MgSO}_{4}\right)$, and chromatographed (silica gel, hexane:EtOAc 1:1) to yield $2(0.94 \mathrm{~g}, 71-77 \%)$ as an orange solid. $\quad$ IR $\left(\mathrm{CCl}_{4}\right) \mathrm{cm}^{-1} 3620$, 2170, 1550, 1250; ${ }^{1} \mathrm{H}$ NMR $\left(\mathrm{CDCl}_{3}\right.$, phenylhydrazine) $\delta(\mathrm{ppm}) 0.972(21 \mathrm{H}, \mathrm{m}), 1.18(6 \mathrm{H}, \mathrm{s})$, $1.24(6 \mathrm{H}, \mathrm{s}), 1.89(4 \mathrm{H}, \mathrm{dd}) ;{ }^{13} \mathrm{C} \mathrm{NMR}\left(\mathrm{CDCl}_{3}\right.$, phenylhydrazine) $\delta(\mathrm{ppm}) 11.47,18.80,25.74$, 26.78, 50.10, 60.01, 60.31, 65.68, 83.94 (Figures 3.1a, 3.1b); MS m/e (relative intensity) 83 (37), 98 (36), 210 (33), 223 (38), 322 (100), 352 (24). HRMS calcd for $\mathrm{C}_{20} \mathrm{H}_{38} \mathrm{SiNO}_{2}$ 352.2727. Found 352.2723.

\section{4-Ethynyltriisopropylsilyl-2,2,6,6-tetramethyl-3,4-dehydro-piperidine-1-oxyl}

Thionyl chloride $(0.5 \mathrm{~g}, 4.2 \mathrm{mmol})$ was added dropwise with stirring to a solution of 2 (1.15 $\mathrm{g}, 3.25 \mathrm{mmol})$ in dry pyridine $(10 \mathrm{~mL})$ while maintaining the temperature below $20^{\circ} \mathrm{C}{ }^{37}$. The reaction was quenched after 3 hours by quickly pouring it onto ice $(50 \mathrm{~g})$, extracted with $\mathrm{CH}_{2} \mathrm{Cl}_{2}(4 \times 30 \mathrm{~mL})$, the organic layer dried $\left(\mathrm{MgSO}_{4}\right)$ and concentrated and chromatographed (silica gel, hexane:EtOAc $11: 1)$ to yield $3(0.59 \mathrm{~g}, 72 \%)$ as an orange oil. IR $\left(\mathrm{CCl}_{4}\right) \mathrm{cm}^{-1}$ 
2950, 2160, 1550, 1250; ${ }^{1} \mathrm{H}$ NMR $\left(\mathrm{CDCl}_{3}\right.$, phenylhydrazine) $\delta(\mathrm{ppm}) 0.83(3 \mathrm{H}, \mathrm{m}), 1.0(18 \mathrm{H}$, m), $1.23(6 \mathrm{H}, \mathrm{s}), 1.30(6 \mathrm{H}, \mathrm{s}), 2.18(2 \mathrm{H}, \mathrm{bs}), 5.79(1 \mathrm{H}, \mathrm{s}) ;{ }^{13} \mathrm{C} \quad \mathrm{NMR}\left(\mathrm{CDCl}_{3}\right.$, phenylhydrazine) $\delta(\mathrm{ppm}) 11.32,18.58,24.65,25.65,43.52,58.9,61.5,89.0,107.68,115.3$, 140.55 (Figures 3.2a, 3.2b); MS m/e (relative intensity) 59(13), 103(17), 219(15), 261(25), 304 (100), 320 (25), 334(15). HRMS calcd for $\mathrm{C}_{20} \mathrm{H}_{36} \mathrm{SiNO} 334.2618$. Found 334.2622.

4-Ethynyl-2,2,6,6-tetramethyl-3,4-dehydro-piperidine-1-oxyl (4): To a solution of 5 $(0.592 \mathrm{~g}, 1.77 \mathrm{mmol})$ in THF $(5 \mathrm{~mL})$ was added a solution of TBAF (1M, $5.3 \mathrm{~mL})$ in wet THF. The solution was stirred at room temperature for $2-3$ hours, brine $(10 \mathrm{ml})$ added, extracted with $\mathrm{CH}_{2} \mathrm{Cl}_{2}(4 \times 20 \mathrm{~mL})$, dried $\left(\mathrm{MgSO}_{4}\right)$, and chromatographed (silica gel, hexane:EtOAc 5:1) to yield $4(0.54 \mathrm{~g}, 60 \%)$ as an orange oil. IR $\left(\mathrm{CCl}_{4}\right) \mathrm{cm}^{-1} 3320,2950$, 2100, 1550; ${ }^{1} \mathrm{H}$ NMR $\left(\mathrm{CDCl}_{3}\right.$, phenylhydrazine) $\delta(\mathrm{ppm}) 1.097(6 \mathrm{H}, \mathrm{s}), 1.16(6 \mathrm{H}, \mathrm{s}), 2.123$ $(2 \mathrm{H}, \mathrm{s}), 2.732(1 \mathrm{H}, \mathrm{s}), 5.837(1 \mathrm{H}, \mathrm{s}) ;{ }^{13} \mathrm{C}$ NMR $\left(\mathrm{CDCl}_{3}\right.$, phenylhydrazine $) \delta(\mathrm{ppm}) 24.66$, 25.75, 43.21, 58.42, 60.89, 75.19, 84.03, 123.5, 142.7 (Figures 3.3a, 3.3b); MS m/e (relative intensity) 91 (100), 105 (69), 133 (100), 148 (79), 178 (25). HRMS calcd for $\mathrm{C}_{11} \mathrm{H}_{16} \mathrm{NO}$ 178.1255. Found 178.1250.

5-(2,2,6,6-tetramethyl-4-ethynylpiperidyl-3-ene-1-oxyl)-uridine (5): Spin-probe nitroxide $4(0.41 \mathrm{~g}, 2.35 \mathrm{mmol})$ was added to a solution of 5-iodo-2 -deoxyuridine 5-IdU (1 g, 2.8 mmol) in $17 \mathrm{ml}$ of dry $\mathrm{N}, \mathrm{N}$-dimethylformamide (DMF). The mixture was placed in the dryice/methanol bath and exposed to a vacuum for 3 times in order to deoxygenate. The addition of copper iodide $(0.67 \mathrm{~g}, 3.5 \mathrm{mmol})$ and tetrakis(triphenyl-phosphine)palladium(0) $(0.42 \mathrm{~g}$, $0.36 \mathrm{mmol}$ ) followed by another deoxygenation cycle. Finally, triethylamine (TEA) ( $0.5 \mathrm{ml}$, 
$3.6 \mathrm{mmol}$ ) was added to a mixture and the reaction was stirred for 12 hours at $25^{\circ} \mathrm{C}{ }^{18}$. The solvents were removed in vacuo, the residue was suspended in $20 \%$ methanol: $\mathrm{CH}_{2} \mathrm{Cl}_{2}$ and filtered through a plug of silica gel. Chromatography (silica gel, $10 \%$ Methanol: $\mathrm{CH}_{2} \mathrm{Cl}_{2}$ ) afforded the thymidine analogue $5(0.34 \mathrm{~g}, 70 \%)$ as a yellow gum. IR $\left(\mathrm{CHCl}_{3}\right) \mathrm{cm}^{-1} 3700$, 3650,3400 (b), 2400, 1740, 1700, 1610, 1510, 1250, 1035; ${ }^{1} \mathrm{H} \quad \mathrm{NMR} \quad\left(\mathrm{CDCl}_{3}\right.$, phenylhydrazine) $\delta(\mathrm{ppm}) 1.16(6 \mathrm{H}, \mathrm{s}), 1.28(6 \mathrm{H}, \mathrm{s}), 2.16(2 \mathrm{H}, \mathrm{m}), 2.35(2 \mathrm{H}, \mathrm{s}), 3.748(1 \mathrm{H}$, m); $3.803(1 \mathrm{H}, \mathrm{m}), 3.99(1 \mathrm{H}, \mathrm{m}), 4.34(1 \mathrm{H}, \mathrm{m}), 6.212(1 \mathrm{H}, \mathrm{t}), 6.256(1 \mathrm{H}, \mathrm{s}), 8.67(1 \mathrm{H}, \mathrm{s})$; (Figure 3.4), UV $\left(\mathrm{CHCl}_{3}\right) \lambda_{\max }$ (log $\varepsilon$ ) 240 (3.86); HRMS calculated for $\mathrm{C}_{20} \mathrm{H}_{26} \mathrm{~N}_{3} \mathrm{O}_{6}$ 404.4437; Found 404.4440.

\section{5-(2,2,6,6-tetramethyl-4-ethynylpiperidyl-3-ene-1-oxyl)-5'-(4,4'-dimethoxyltriphenyl)-}

uridine (6): The thymidine analogue $5(1.3 \mathrm{~g}, 3.2 \mathrm{mmol})$ was mixed with 4,4dimethoxytriphenylmethyl chloride and dissolved in dry pyridine $(10 \mathrm{ml})$. The reaction was stirred for 2 hours under $\mathrm{N}_{2}$ at $25^{\circ} \mathrm{C}$ and) quenched by the addition of methanol $(5 \mathrm{~mL})$, concentrated in vacuo and purified by column chromatography (silica gel, 5\% Methanol: $\left.\mathrm{CH}_{2} \mathrm{Cl}_{2}\right)$ to give the monoprotected nucleoside $6(1.95 \mathrm{~g}, 80 \%)$ as yellow solid. IR $\left(\mathrm{CCl}_{4}\right) \mathrm{cm}^{-1}$ 3695, 3620, 3395 (b), 3020, 3010, 2990, 2402,1715, 1705, 1610, 1510, 1455, 1249, 1035; ${ }^{1} \mathrm{H}$ NMR $\left(\mathrm{CDCl}_{3}\right.$, phenylhydrazine) $\delta(\mathrm{ppm}) 1.60(6 \mathrm{H}, \mathrm{d}), 1.69(6 \mathrm{H}, \mathrm{d}), 2.66(2 \mathrm{H}$, s), $2.76(1 \mathrm{H}, \mathrm{m}), 3.01(1 \mathrm{H}, \mathrm{m}) ; 3.719(1 \mathrm{H}, \mathrm{d}), 3.816(1 \mathrm{H}, \mathrm{d}), 3.95(1 \mathrm{H}, \mathrm{s}), 4.065(3 \mathrm{H}, \mathrm{s})$, $4.075(3 \mathrm{H}, \mathrm{s}), 4.47(1 \mathrm{H}, \mathrm{m}), 5.081(1 \mathrm{H}, \mathrm{d}), 5.781(1 \mathrm{H}, \mathrm{s}), 6.511(1 \mathrm{H}, \mathrm{s}), 9.271(1 \mathrm{H}, \mathrm{s})$ (Figure 3.5); UV $\left(\mathrm{CHCl}_{3}\right) \lambda_{\max }(\log \varepsilon) 257$ (s, 4.03), 276 (3.91), 308 (4.00); HRMS calculated for $\mathrm{C}_{41} \mathrm{H}_{44} \mathrm{~N}_{3} \mathrm{O}_{8}$ 706.8023. Found 706.8100. 


\section{5-(2,2,6,6-tetramethyl-4-ethynylpiperidyl-3-ene-1-oxyl)-5' -(4,4'-dimethoxyltriphenyl)-}

uridine phosphoramidite (7): To a solution of $6(0.29 \mathrm{~g}, 0.41 \mathrm{mmol})$ in $2.5 \mathrm{ml}$ of dry $\mathrm{CH}_{2} \mathrm{Cl}_{2}$ were added triethylamine $(143 \mu \mathrm{l}, 1.026 \mathrm{mmol})$ and 2-cyanoethyl-diisopropylchlorophosphoramidite $(107 \mu \mathrm{l}, 0.45 \mathrm{mmol})$. The reaction was stirred for 1 hour at $25^{\circ} \mathrm{C}$ and formation of the product was monitored by TLC. A second portion of 2-cyanoethyldiisopropylchloro-phosphoramidite was then added (39 $\mu 1,0.17 \mathrm{mmol})$ and stirred another hour. The reaction mixture was concentrated in vacuo, tetrahydrofuran:benzene $(1: 4,2 \mathrm{~mL})$ added and stirred for $10 \mathrm{~min}$. The precipitate was removed by filtration, the filtrate was concentrated in vacuo, and the residue twice coevaporated with benzene (3 $\mathrm{ml})$. Chromatography on silica gel (Petroleum-ether:EtOAc:TEA 50:50:1) afforded phosphoramidite $7(0.32 \mathrm{~g}, 86 \%)$ as yellow powder. IR $\left(\mathrm{CHCl}_{3}\right) \mathrm{cm}^{-1} 3695,3610,3020,3005$, 2985, 2450, 1740, 1580, 1506, 1240, 1190, 1175, 1030, 920; ${ }^{1} \mathrm{H}$ NMR $\left(\mathrm{CD}_{3} \mathrm{CN}\right.$, phenylhydrazine) $\delta(\mathrm{ppm}) 1.15 / 1.17\left(6 \mathrm{H}, \mathrm{s},\left(\mathrm{CH}_{3}\right)_{2}\right), 1.33 / 1.35\left(6 \mathrm{H}, \mathrm{s},\left(\mathrm{C}_{\mathrm{H}_{3}}\right)_{2}\right), 1.250 / 1.251$ (12H, s, CH( $\left.\left.\underline{\mathrm{CH}}_{3}\right)_{2}\right), 2.26\left(2 \mathrm{H}, \mathrm{m}, \mathrm{CH}_{2}\right), 2.26$ (1H, m, H-2'), 2.56 (1H, m, H-2"), 2.63/2.73 $\left(1 \mathrm{H}, \mathrm{t}, \mathrm{J}=6 \mathrm{~Hz}, \underline{\mathrm{H}}_{2} \mathrm{CN}\right), 3.56(1 \mathrm{H}, \mathrm{m}, \mathrm{H}-5$ '), $3.57(1 \mathrm{H}, \mathrm{m}, \mathrm{H}-5 "), 3.67 / 3.71(1 \mathrm{H}, \mathrm{m}$, $\left.\mathrm{C} \underline{\mathrm{H}}\left(\mathrm{CH}_{3}\right)_{2}\right), 3.74(1 \mathrm{H}, \mathrm{m}, \mathrm{POC} \underline{\mathrm{H}}), 3.84\left(6 \mathrm{H}, \mathrm{s}, \mathrm{OCH}_{3}\right), 3.92(1 \mathrm{H}, \mathrm{m}, \mathrm{POC} \underline{\mathrm{H}}), 4.23(1 \mathrm{H}, \mathrm{m}, \mathrm{H}-$ 4'), $4.84\left(1 \mathrm{H}, \mathrm{m}, \mathrm{H}-3^{\prime}\right), 5.72(1 \mathrm{H}, \mathrm{bs}, \mathrm{CH}=\mathrm{C}), 6.28\left(1 \mathrm{H}, \mathrm{m}, \mathrm{H}-1^{\prime}\right), 6.85(4 \mathrm{H}, \mathrm{d}, \mathrm{J}=8$, ArH3,3',5,5'), 7.26 (4H, d, J=8, ArH-2,2',6,6'), 7.26-7.39 (4H, m, ArH-2",3",5",6"), 7.42 (1H, dd, J=2, $8.5 \mathrm{~Hz}$, ArH-4"), 8.4 (1H, bs, NH), 8.78/8.81 (1H, s, H-6) (Figure 3.6); UV $\left(\mathrm{CHCl}_{3}\right) \lambda_{\max }$ $\left(\log \varepsilon\right.$ ) 240 (3.86), 275 (3.53), 302 (3.51); HRMS calculated for $\mathrm{C}_{50} \mathrm{H}_{62} \mathrm{~N}_{5} \mathrm{O}_{9} \mathrm{P} 907.0239$. Found 907.0135. 


\subsubsection{Oligonucleotide synthesis}

Large scale $(10-20 \mu \mathrm{mol})$ synthesis was conducted on modified ABI 430A protein synthesizer. The oligonucleotides $\mathrm{T}_{15}, \mathrm{~A}_{15}$, and ${ }^{6 \mathrm{sp}} \mathrm{T}_{15}\left(\mathrm{~T}_{7}{ }^{6 \mathrm{sp}} \mathrm{TT}_{7}\right)$, used in this study, were synthesized using the solid-phase phosphoramidite protocol. The synthetic steps were detritylation, coupling, capping, and oxidation. The oligonucleotides (10-20 uM scale) were cleaved off the resin by treatment with concentrated $\mathrm{NH}_{4} \mathrm{OH}(28-30 \%, 12 \mathrm{ml})$ at room temperature for 1 hour and filtered through $2 \mu \mathrm{m}$ filter disk. The cleavage of protecting groups for $\mathrm{A}_{15}$ oligonucleotide was accomplished by heating the filtrate at $55^{\circ} \mathrm{C}$ for 20 hours and then dried down in a SpeedVac. Final purification of oligomers was achieved by (FPLC) using a Bio-Rad TSK DEAE-5-PW column. $\mathrm{T}_{15}$ was purified under isocratic conditions with a gradient (53\% B, Buffer A: $10 \mathrm{mM} \mathrm{NaOH}, \mathrm{pH}$ 11.8, Buffer B: $10 \mathrm{mM} \mathrm{NaOH}, 1 \mathrm{mM} \mathrm{NaCl}$, pH 11.8 , flow rate $7 \mathrm{ml} / \mathrm{min}$, detecting at $\lambda=260 \mathrm{~nm}$ wavelength). $\mathrm{A}_{15}$ and ${ }^{6 \mathrm{sp}} \mathrm{T}_{15}$ were purified with a gradient (20-26\% B) over 45 min and (40-55\% B) over 90 min, respectively. The oligomers were desalted with reverse phase Waters Sep-Pack (C-18) cartridges by 60\% $\mathrm{MeOH} /$ water elution. 
a)

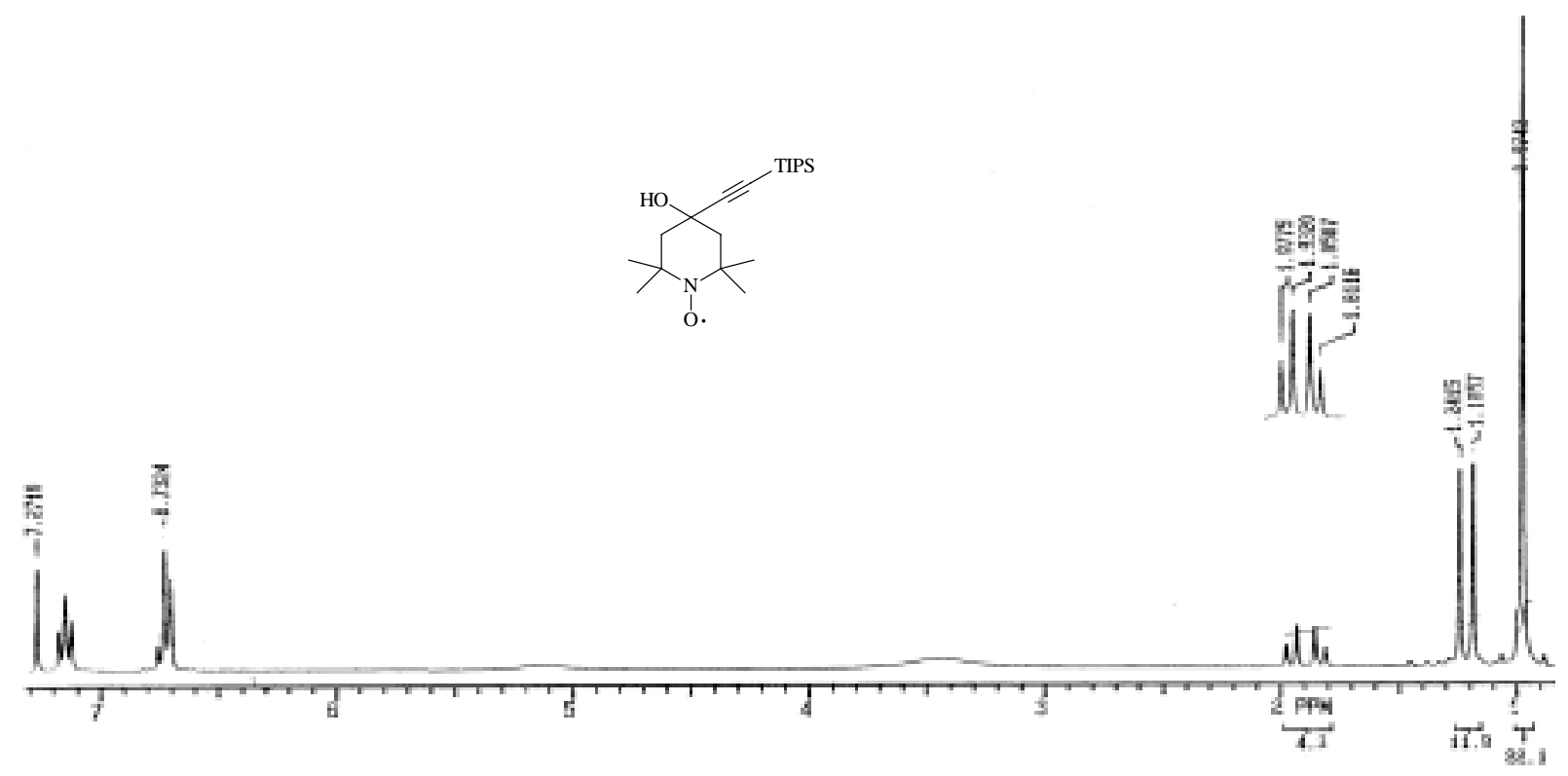

b)

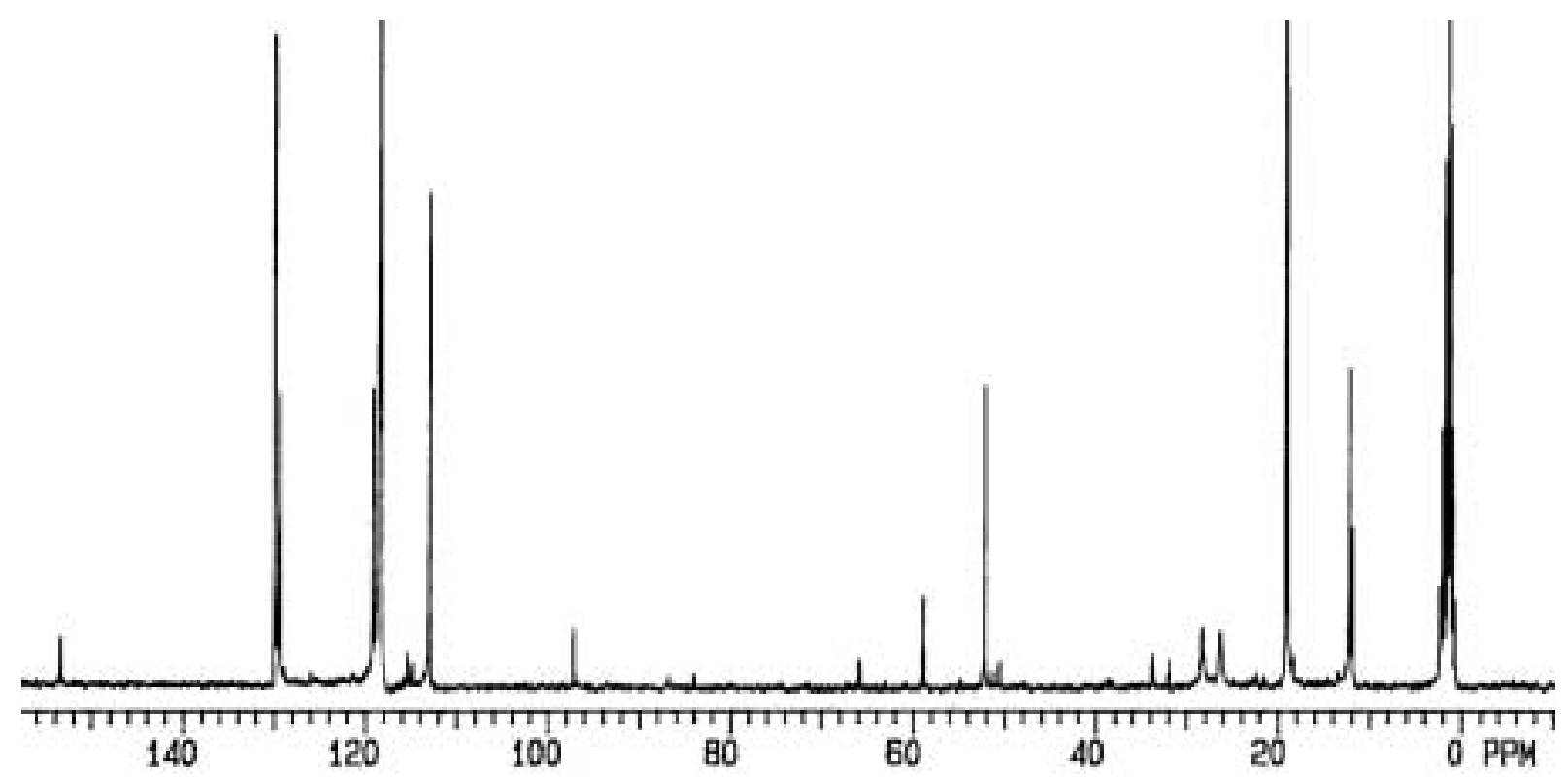

Figure 3.1. a) ${ }^{1} \mathrm{H}$ NMR and b) ${ }^{13} \mathrm{C}$ NMR spectra of 4-Ethynyltriisopropylsilyl-4-hydroxy-2,2,6,6tetramethyl-piperidine-1-oxyl (2). The sample was prepared in $\mathrm{CDCl}_{3}$, and reduced with phenylhydrazine. 
a)

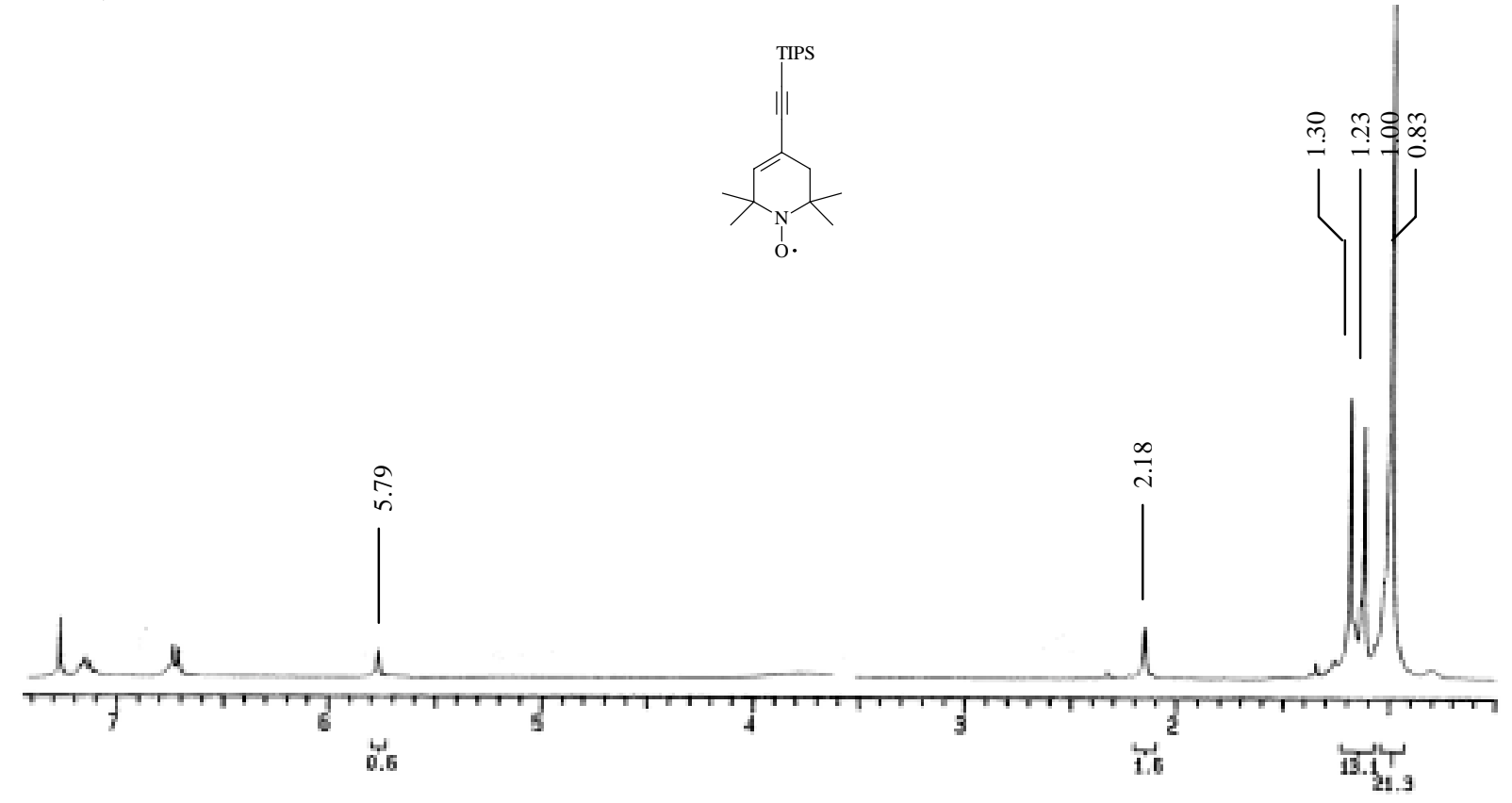

b)

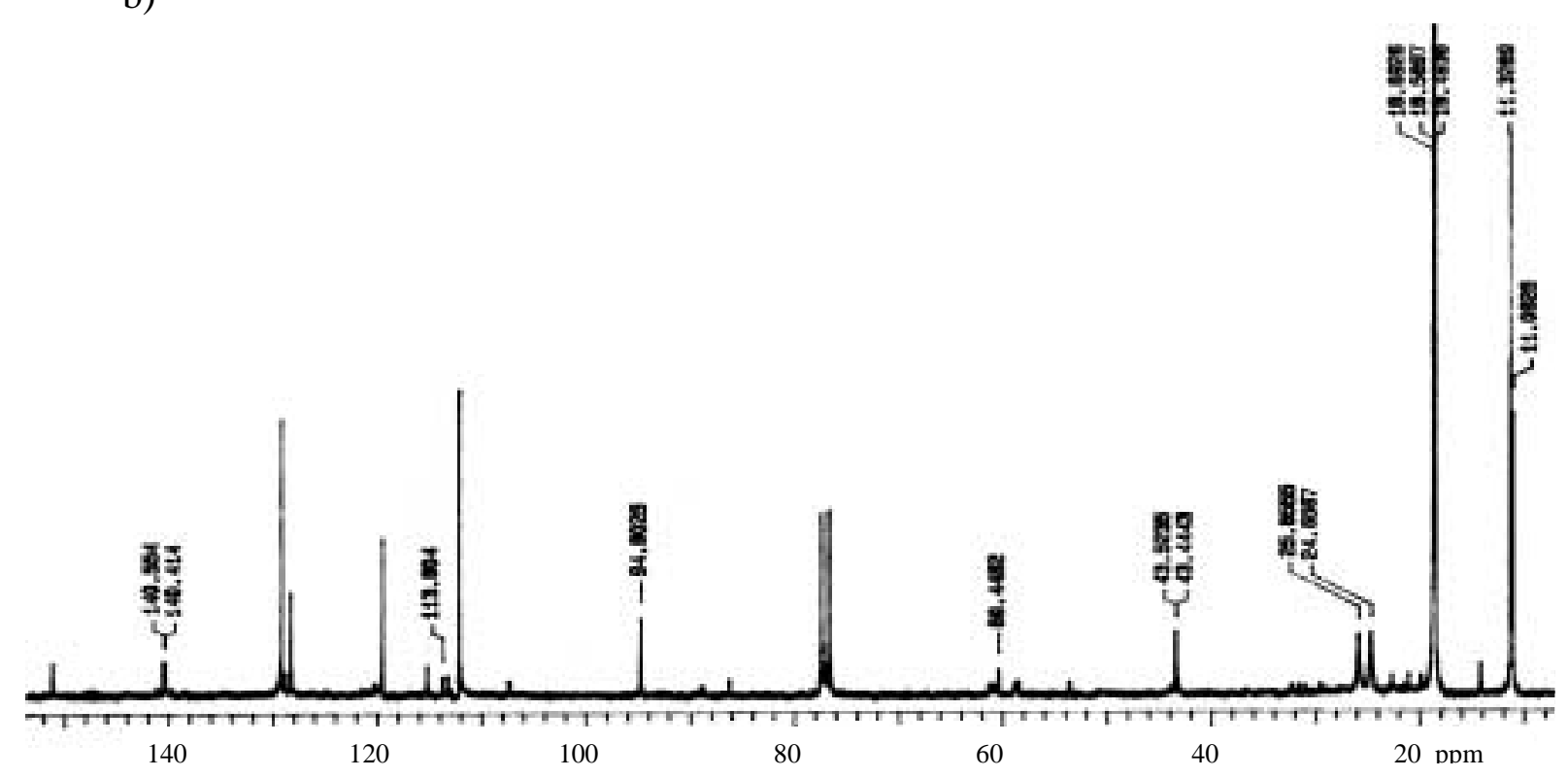

Figure 3.2. a) ${ }^{1} \mathrm{H}$ NMR and b) ${ }^{13} \mathrm{C}$ NMR spectra of 4-Ethynyltriisopropylsilyl-2,2,6,6-tetramethyl-3,4dehydro-piperidine-1-oxyl (3). The sample was prepared in $\mathrm{CDCl}_{3}$, and reduced with phenylhydrazine. 
a)
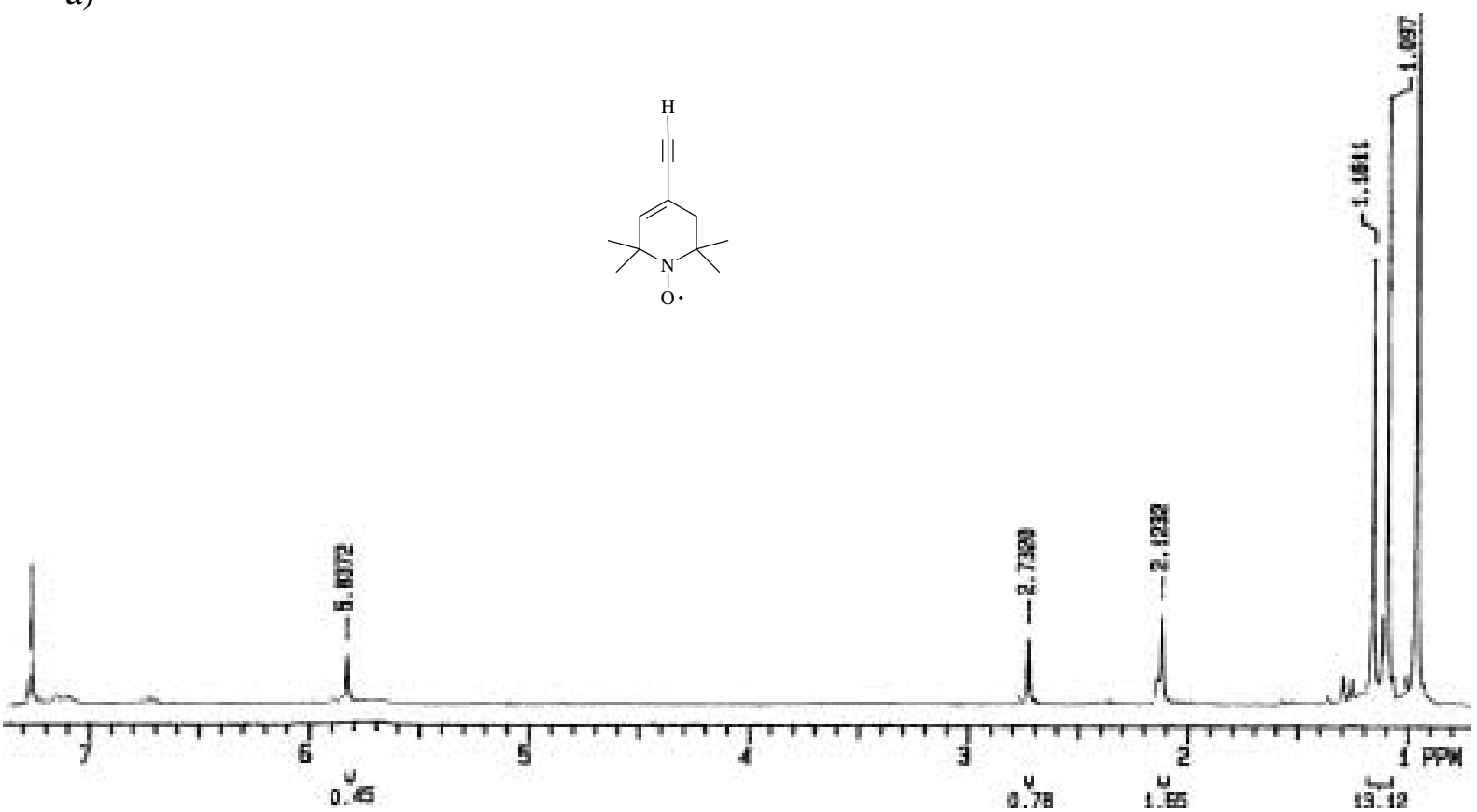

b)

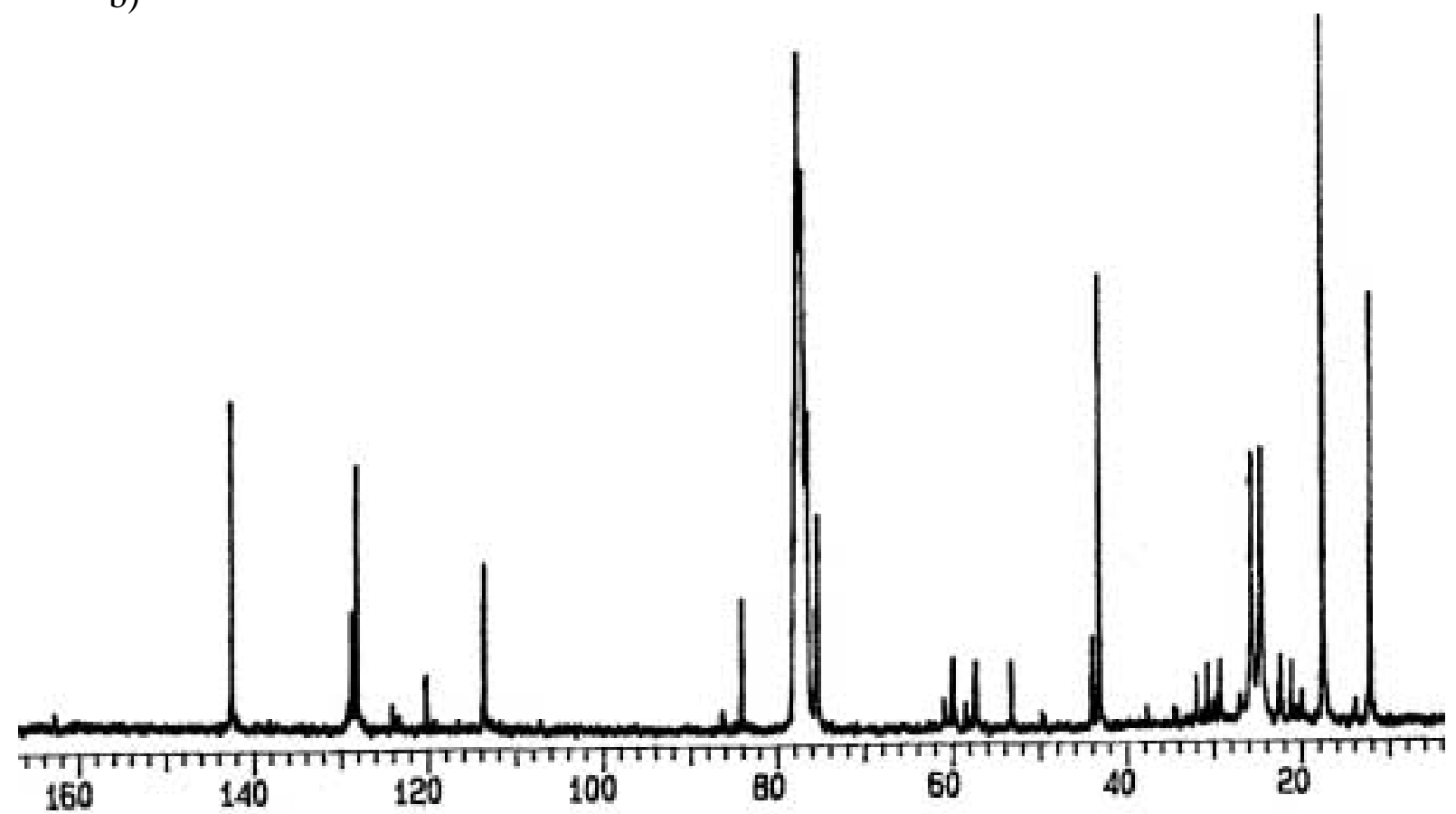

Figure 3.3. a) ${ }^{1} \mathrm{H}$ NMR and b) ${ }^{13} \mathrm{C}$ NMR spectra of 4-Ethynyl-2,2,6,6-tetramethyl-3,4-dehydropiperidine-1-oxyl (4). The sample was prepared in $\mathrm{CDCl}_{3}$, and reduced with phenylhydrazine. 


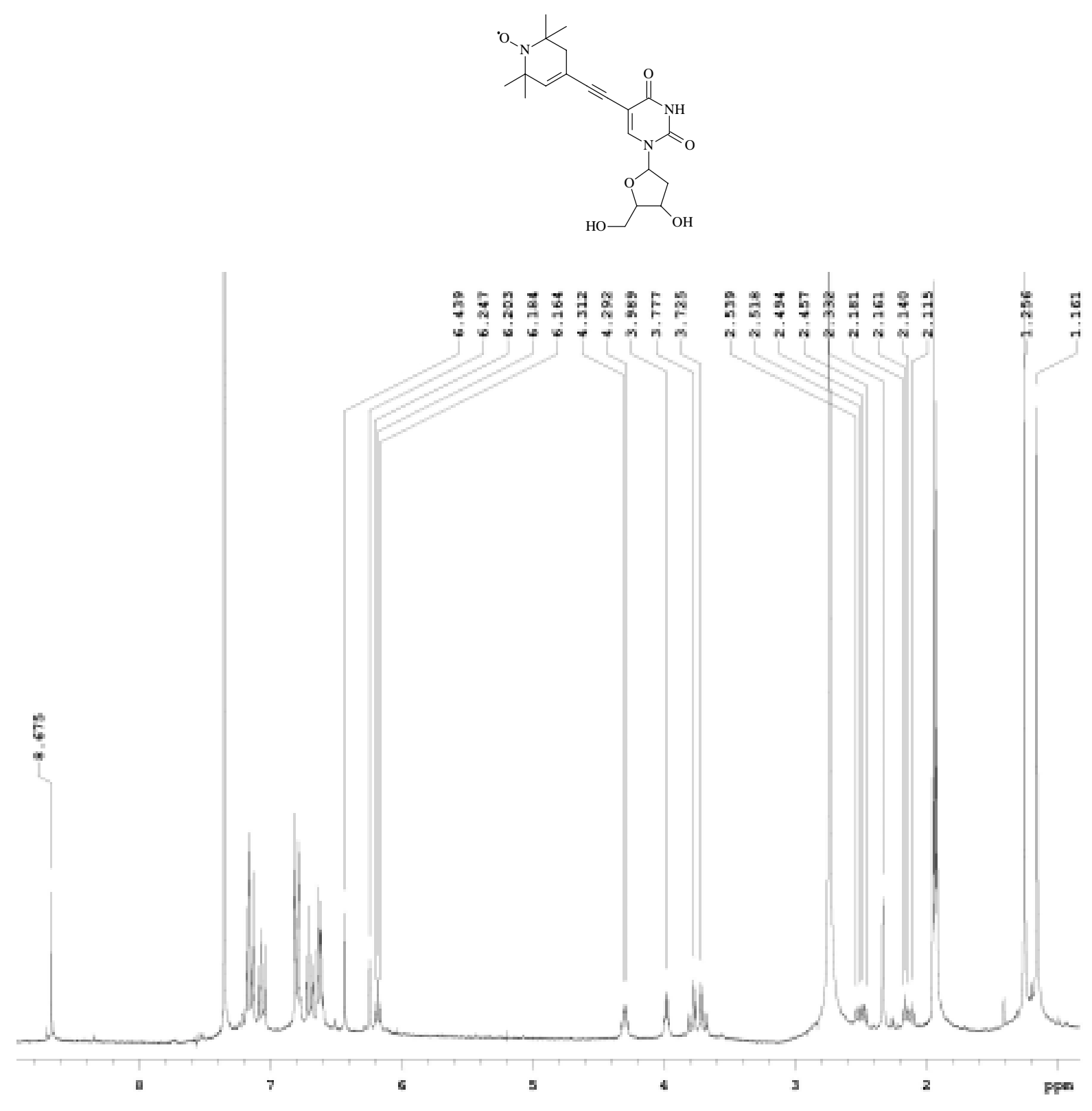

Figure 3.4. ${ }^{1} \mathrm{H}$ NMR spectrum of 5-(2,2,6,6-tetramethyl-4-ethynylpiperidyl-3-ene-1-oxyl)-uridine (5). The sample was prepared in $\mathrm{CD}_{3} \mathrm{CN}$, and reduced with phenylhydrazine. 


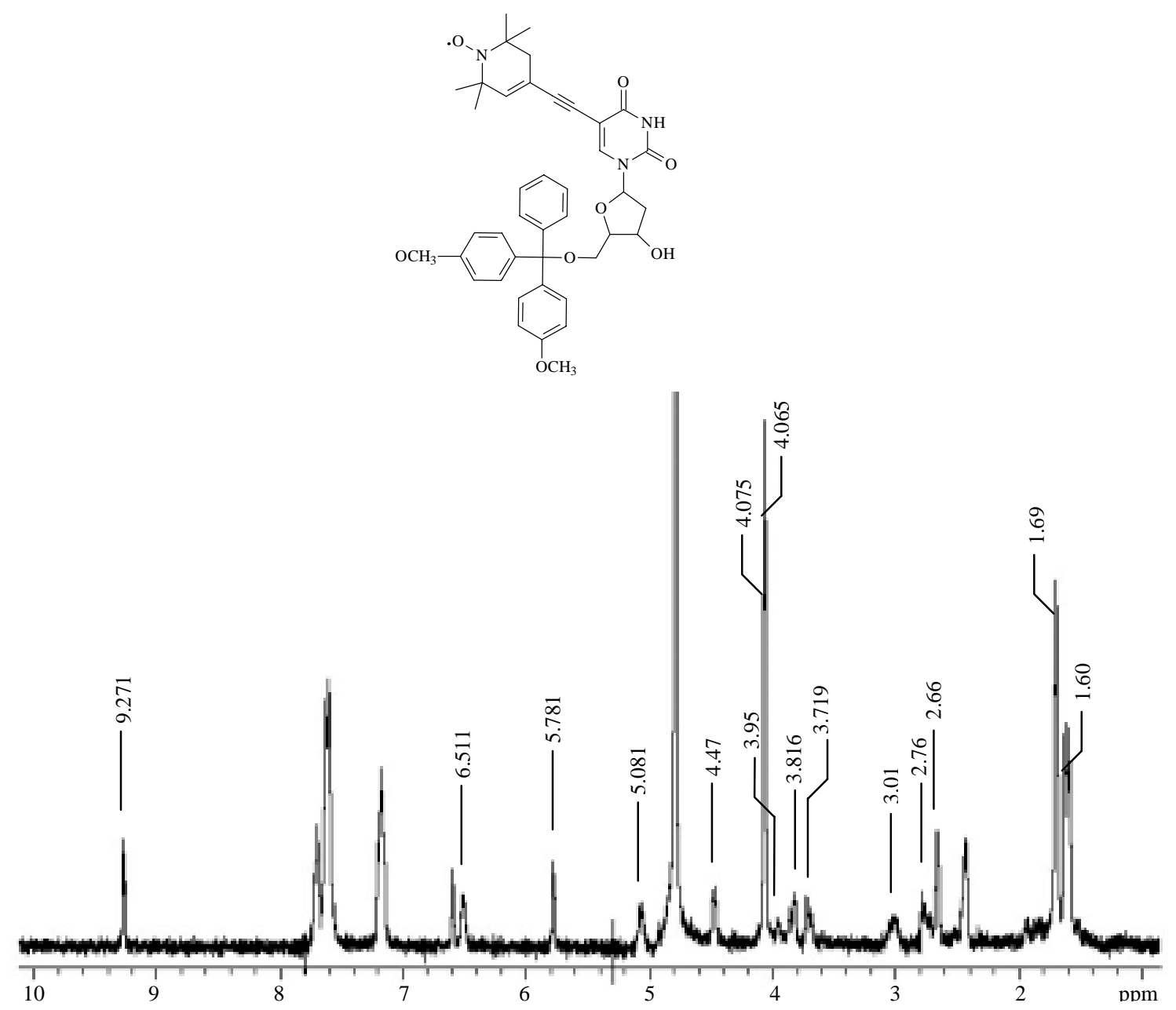

Figure 3.5. ${ }^{1} \mathrm{H}$ NMR spectrum of 5-(2,2,6,6-tetramethyl-4-ethynylpiperidyl-3-ene-1-oxyl)-5'-(4,4'dimethoxyltriphenyl)-uridine (6). The sample was prepared in $\mathrm{CD}_{3} \mathrm{CN}$, and reduced with phenylhydrazine. 

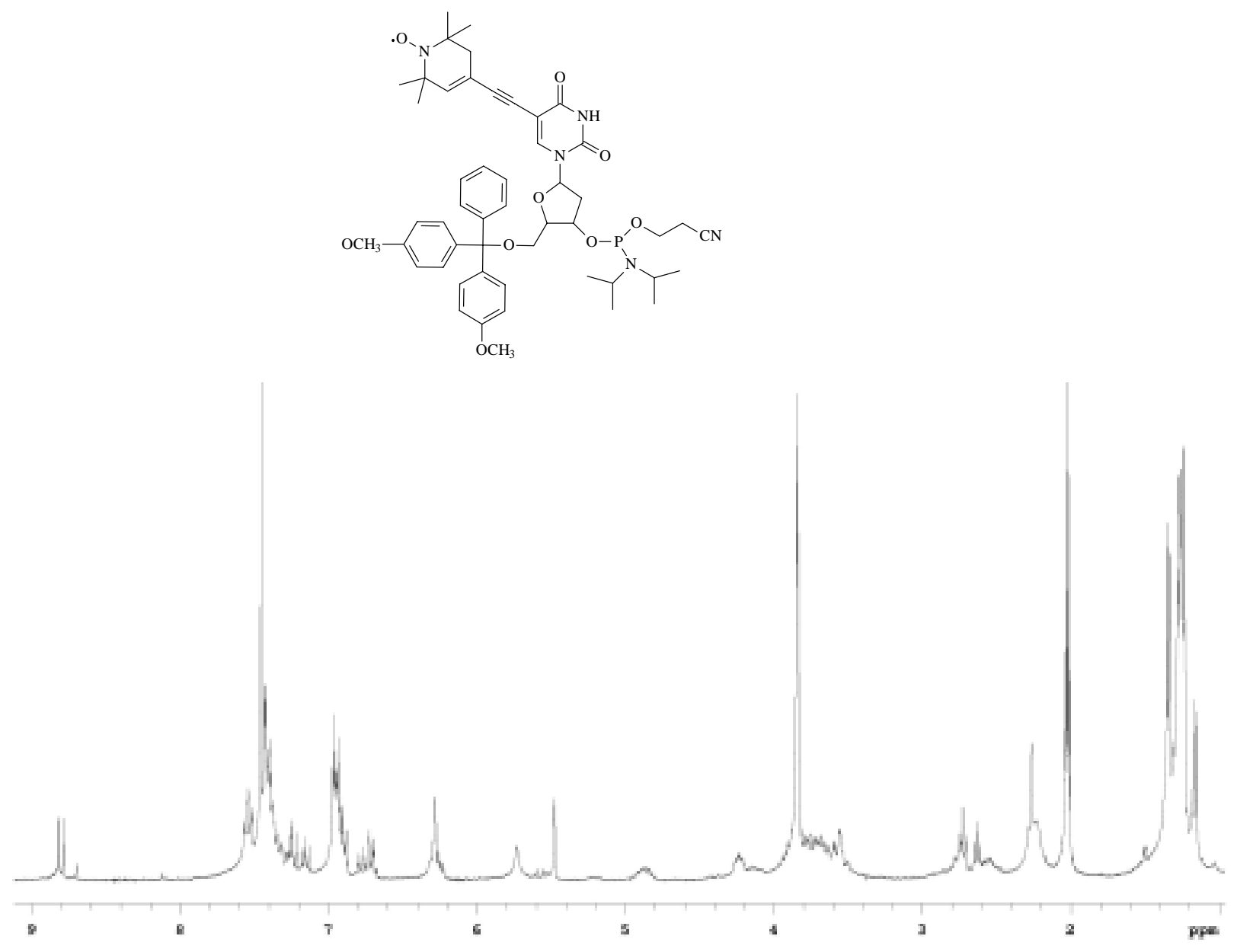

Figure 3.6. ${ }^{1} \mathrm{H}$ NMR spectrum of 5-(2,2,6,6-tetramethyl-4-ethynylpiperidyl-3-ene-1-oxyl)-5'-(4,4'dimethoxyltriphenyl)-uridine phosphoramidite (7). The sample was prepared in $\mathrm{CD}_{3} \mathrm{CN}$, and reduced with phenylhydrazine. 


\subsection{Sample preparation and analysis}

The following sequences of DNAs were used in the current work: double stranded (ds) $\mathrm{A}_{15}: \mathrm{T}_{15}, \mathrm{~A}_{15}:{ }^{6 \mathrm{sp}} \mathrm{T}_{15}$ and triple stranded (triplex (tx)) DNAs $\mathrm{T}_{15}-\mathrm{A}_{15}: \mathrm{T}_{15},{ }^{6 \mathrm{sp}} \mathrm{T}_{15}-\mathrm{A}_{15}: \mathrm{T}_{15}$. Here the Watson-Crick base pairing is indicated by a colon (:) and Hoogsteen base-paired strand by a hyphen (-). All DNA samples were made up in phosphate buffer. Duplex formation was achieved by mixing equivalent amounts of $\mathrm{T}_{15},{ }^{6 \mathrm{sp}} \mathrm{T}$, and $\mathrm{A}_{15}$ and heating to $90^{\circ} \mathrm{C}$ for 30 min and then slowly cooling to room temperature. Triple stranded DNAs are prepared by first forming Watson-Crick duplex $\mathrm{A}_{15}: \mathrm{T}_{15}$ in the same manner as described above. In order to have triplex formation, $\mathrm{MgCl}_{2}$ was added to the buffer of the third strand, ${ }^{6 \mathrm{sp}} \mathrm{T}_{15}$. Then, the third strand was added to the duplex and the mixture was incubated at $4^{\circ} \mathrm{C}$ for $24 \mathrm{~h}$.

NMR spectra were obtained on a Varian Gemini 300 broadband spectrometer. Methylene chloride was dried by distillation from phosphorus pentoxide. Triethylamine, pyridine and THF were dried by distillation from LAH. Dimethylformamide was purified by distillation from barium oxide. Samples were either dissolved in $\mathrm{CDCl}_{3}$ and reduced with phenylhydrazine or in 1:1 $\mathrm{D}_{2} \mathrm{O}$ :acetone- $\mathrm{d}_{6}$ and reduced with sodium dithonite to convert the nitroxide to the hydroxylamine prior to NMR data acquisition ${ }^{38}$. Thus, the reported proton and carbon spectra refer to the hydroxylamine derived from the corresponding nitroxides (27). Mass spectra (MS) were recorded on an Agilent 5973N (low resolution), a Finnigan MAT 90 (high resolution) or Bruker MALDI-TOF (model \#*******).

UV-monitored melting temperature experiments were conduced at $260 \mathrm{~nm}$ using a Cary 300 spectrometer on both unmodified and spin-labeled duplexes and triplexes. Sample concentrations were approximately $0.5 \mathrm{OD}$. Spectra were recorded at $5-90^{\circ} \mathrm{C}$ at a rate of $0.25^{\circ} \mathrm{C} / \mathrm{min}$. 
The CD spectra were recorded on an AVIV Model 62A CD spectrometer. Solutions were approximately $25 \mu \mathrm{M}$ in duplex and triplex. Final sample volume was $400 \mu \mathrm{L}$. Spectra were recorded as function of temperature every $5^{\circ} \mathrm{C}$ from $30-75^{\circ} \mathrm{C}$ for double stranded DNA and from $5-75^{\circ} \mathrm{C}$ for triple stranded DNAs.

Continuous-wave (CW) EPR spectra were obtained on a Brucker EMX X-band or Varian E-12 spectrometer. Samples were dissolved in phosphate buffer and loaded either in glass capillaries $(10 \mu \mathrm{l})$ or in a flat cell at DNA concentrations of ca. $2 \mathrm{mM}$ in single stranded, duplex or triplex DNA. Spectra were acquired at either $0^{\circ} \mathrm{C}$ or $25^{\circ} \mathrm{C}$ under nearly identical instrumental settings: receiver gain $6.32 \cdot 10^{3}$, sweep width $100.0 \mathrm{G}$, modulation amplitude $1.00 \mathrm{G}$, modulation frequency $100.00 \mathrm{kHz}$, microwave power $20 \mathrm{~mW}$, time constant 5.120 ms. 


\subsection{Results and Discussion}

The full synthetic path of phosphoramidite 7 is shown in Figure 3.7 and it begins with the synthesis of the compound $\mathbf{2}$. The commercially available nitroxide compound $\mathbf{1}$ was condensed<smiles>CC(C)CCCCCC#CC1=CC(C)(C)N(OC(C)C)C(C)(C)C1</smiles><smiles>C#CC1=CC(C)(C)N([O])C(C)(C)C1</smiles>

4<smiles>O=c1[nH]c(=O)n(C2CC(O)C(CO)O2)cc1I</smiles>

5-IdU<smiles>[R20]CC1OC(n2cc(C#CC3=CC(C)(C)N([O])C(C)(C)C3)c(=O)[nH]c2=O)CC1O[R2]</smiles>

e $5 \mathrm{R}=\mathrm{R}^{\prime}=\mathrm{H}$

$6 \mathrm{R}=\mathrm{DMTr}, \mathrm{R}^{\prime}=\mathrm{H}$

$7 \mathrm{R}=\mathrm{DMTr}$, $\mathrm{R}^{\prime}=\mathrm{N}(i-\mathrm{Pr})_{2} \mathrm{P}-\mathrm{OCH}_{2} \mathrm{CH}_{2} \mathrm{CN}$

Figure 3.7. Synthetic scheme followed for the preparation of the new spin-label phosphoramidite 7. Reaction conditions: a) TIPS $-\equiv-\mathrm{Li}, \mathrm{THF},-78^{\circ} \mathrm{C}$, b) $\mathrm{SOCl}_{2}$, pyridine, $\left.\left.0^{\circ} \mathrm{C}, \mathrm{c}\right) \mathrm{TBAF}, \mathrm{THF}, \mathrm{d}\right)$ $\left.\mathrm{Pd}\left[\mathrm{P}\left(\mathrm{C}_{6} \mathrm{H}_{5}\right)_{3}\right)\right]_{4}, \mathrm{CuI}$, TEA, DMF, e) trityl-chloride, TEA, $\mathrm{CH}_{2} \mathrm{Cl}_{2}$, f) $\mathrm{Cl}-\mathrm{P}\left(\mathrm{OCH}_{2} \mathrm{CH}_{2} \mathrm{CN}\right)\left(\mathrm{N}(i-\mathrm{Pr})_{2}\right), \mathrm{TEA}$, $\mathrm{CH}_{2} \mathrm{Cl}_{2}$ ). 
with the triisopropylsilylacetylene lithium salt in dry THF to afford the acetylenic alcohol 2. The second step is the elimination of alcohol to the enyne $\mathbf{3}$, and the triisoproylsilyl group then removed by treatment with tetrabutylammonium fluoride in wet THF to give 4 . Next, 4 was coupled to 5-iodouridine 5-dIU to give the nucleoside 5. Attachment of the protecting group to the 5'-OH end of the nucleoside and phosphorylation of the 3'-OH are necessary for automated DNA synthesis. These steps were accomplished under standard conditions and yielded the phosphoramidite 7 .

Oligonucleotide synthesis and purification. Oligonucleotides were prepared using the phosphoramidite methodology on controlled pore glass (CPG) and the standard reaction cycle of deprotection, coupling, capping and oxidation. The coupling efficiencies were $98-99 \%$ for both unmodified and modified oligonucleotides base on the optical densities (OD) of the products. However, FPLC of the ${ }^{6 \mathrm{sp}} \mathrm{T}_{15}$ modified oligonucleotide showed the presence of three peaks, corresponding to three different oligonucleotides. The major product was the oligonucleotide with an intermediate retention time of $42.0 \mathrm{~min}$ for ${ }^{6 \mathrm{sp}} \mathrm{T}_{15}$, and it was collected for further analysis. This product was the 15-mer oligonucleotide containing the spin-label and gave an ESR signal. The oligonucleotide with the longer retention time of $44.7 \mathrm{~min}$ did not give an EPR signal by itself, but the addition of hydrogen peroxide did produce the sample that became EPR active. This implies that during the DNA synthesis some of the nitroxide gets reduced to its hydroxylamine derivative.

The oligonucleotide with the shorter retention time of 40.8 min did not give an EPR signal regardless of whether the hydrogen peroxide was added or not. To investigate further, the mass spectral analysis of the latter oligonucleotide was done. It revealed that it had a 
molecular weight of approximately 30 less than expected for the spin-labeled 15 -mer. This suggests that this oligonucleotide has lost the NO group. To investigate even further, we synthesized oligonucleotides of different length. The oligonucleotide synthesis was stopped after the addition of the spin label after the eighth base, $T_{7}{ }^{6 \mathrm{sp}} \mathrm{T}$, after the twelfth base, $\mathrm{T}_{7}$ ${ }^{6 s p} \mathrm{TT}_{4}$ and after the fifteenth base, $\mathrm{T}_{7}{ }^{6 \mathrm{sp}} \mathrm{TT}_{7}$. Then, the sequences were cleaved from the resin, and examined by FPLC and EPR. FPLC of the 8-mer showed mainly one oligonucleotide that was EPR active. Here the spin-labeled base was the terminal one. As the number of synthesis cycles continued to twelve and then fifteen, increasing amounts of the oligonucleotide, which had lost the NO group appeared. This kind of reaction may occur during the iodine oxidation step. It is known that nitroxides are sensitive to oxidation by halogens and results in the formation of a nitrone, which can subsequently decompose with the loss of $\mathrm{NO}^{39}$. Thus, oxidants, other then iodine, may improve the purity of spin labeled oligonucleotides prepared by automated DNA synthesis.

Thermal Denaturation studies: Thermal denaturation curves of unmodified and spinlabeled modified double stranded DNAs (ds DNA) and triplex DNAs (tx DNA) were measured. The ds DNA curves were obtained on samples made up in $10 \mathrm{mM}$ phosphate buffer $(\mathrm{pH}$ 7.4) and $100 \mathrm{mM}$ sodium chloride to which magnesium chloride was added. Representative curves for duplexes at $25 \mathrm{mM} \mathrm{MgCl} 2$ salt concentration are shown in Figure 3.8a. Both curves have quite similar shape and there are no significant differences in the $T_{m}$ values measured between the unmodified and modified ds DNAs (Table 3.1).

The thermal denaturation curves of the tx DNAs $\left({ }^{*} \mathrm{~T}_{15}-\mathrm{A}_{15}: \mathrm{T}_{15}\right.$, where ${ }^{*} \mathrm{~T}$ is the third $\mathrm{T}_{15}$, or ${ }^{6 s p} \mathrm{~T}_{15}$ strand, and $\mathrm{A}_{15}: \mathrm{T}_{15}$ is the Watson-Crick base paired ds DNA) where also 
measured and selected plots are shown in Figure 3.8b. Two $\mathrm{T}_{\mathrm{m}}$ values are observed for tx DNAs: the first corresponds to the melting of the third strand to give a single stranded oligonucleotide $\left(\mathrm{T}_{15}\right.$, or $\left.{ }^{6 s \mathrm{p}} \mathrm{T}_{15}\right)$ and a ds DNA $\left(\mathrm{A}_{15}: \mathrm{T}_{15}\right)$. The second $\mathrm{T}_{\mathrm{m}}$ is observed for the melting of the Watson-Crick duplex. The $\mathrm{T}_{\mathrm{m}}$ for the triplex melting is not always observable due to the low temperatures typically observed for this process and because the small difference in absorbance between the triplex+duplex and the triplex strand ${ }^{40}$. However, at relatively high concentrations of $\mathrm{MgCl}_{2}$, which are the conditions known to stabilize tx DNA structure ${ }^{41}$, two transitions can be observed for all the triplexes. The data for all magnesium chloride concentrations for both duplexes and triplexes is shown in Table 3.1. As can be seen from the table the Tm values measured here, for all duplexes and triplexes, are not significantly different from one another under a given set of conditions.

\begin{tabular}{|c|c|c|c|c|}
\hline & $\mathbf{A}_{\mathbf{1 5}} \mathbf{T}_{\mathbf{1 5}}$ & $\mathbf{A}_{\mathbf{1 5}}:{ }^{\mathbf{6 s}} \mathbf{T}_{\mathbf{1 5}}$ & $\mathbf{T}_{\mathbf{1 5}}-\mathbf{A}_{\mathbf{1 5}}: \mathbf{T}_{\mathbf{1 5}}$ & ${ }^{{ }^{\mathbf{s p}} \mathbf{T}_{\mathbf{1 5}}-\mathbf{A}_{\mathbf{1 5}}: \mathbf{T}_{\mathbf{1 5}}}$ \\
\hline \hline${ }^{\mathrm{a}} \mathbf{T}_{\mathrm{m}}$ & 24 & 22 & $\mathrm{ND}$ & $\mathrm{ND}$ \\
\hline${ }^{\mathrm{b}} \mathrm{T}_{\mathrm{m}}$ & 36 & 35 & $(20) 41$ & $(18) 41$ \\
\hline${ }^{\mathrm{c}} \mathrm{T}_{\mathrm{m}}$ & 47 & 45 & $(24) 50$ & (24) 49 \\
\hline
\end{tabular}

aDNA melting temperatures were measured on solutions of approximately 0.5 OD in sodium phosphate buffer (10 mM, pH 7.4) and sodium chloride (100 mM). ${ }^{\mathrm{b}}$ Samples also contained $10 \mathrm{mM} \mathrm{MgCl} 2$. ${ }^{\mathrm{c} S}$ Samples also contained $50 \mathrm{mM} \mathrm{MgCl}_{2}$.

Table 3.1. UV melting temperatures for the unmodified and spin-labeled modified duplexes and triplexes. 

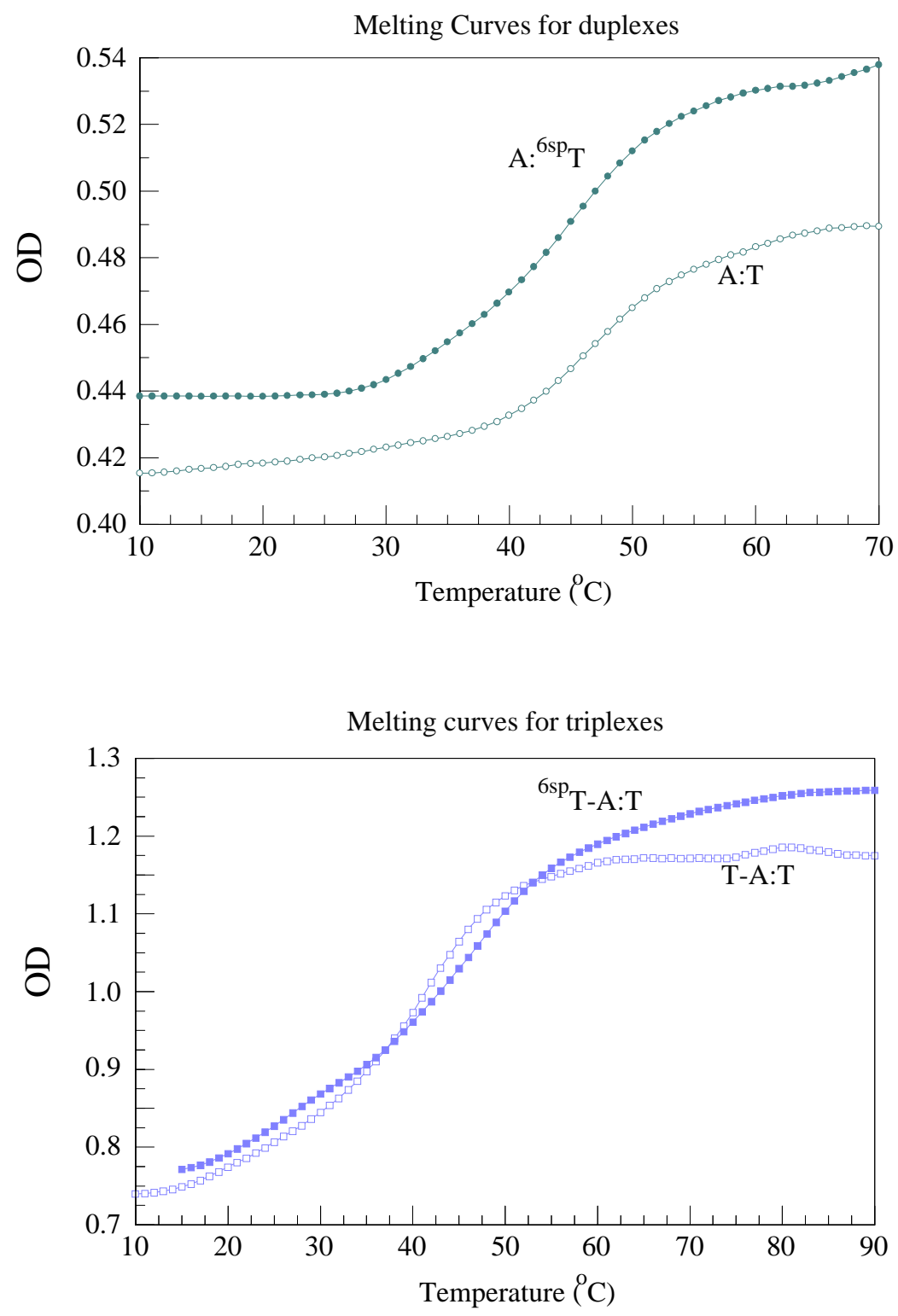

Figure 3.8. Thermal denaturation curves for a) $\mathrm{A}_{15}: \mathrm{T}_{15}(\bullet)$, and $\mathrm{A}_{15}:{ }^{6 \mathrm{sp}} \mathrm{T}_{15}(\mathrm{O})$ duplexes at $50 \mathrm{mM} \mathrm{MgCl} 2$ salt concentration and b) $\mathrm{T}_{15}-\mathrm{A}_{15}: \mathrm{T}_{15}(\boldsymbol{\square})$ and ${ }^{6 \mathrm{sp}} \mathrm{T}_{15}-\mathrm{A}_{15}: \mathrm{T}_{15}(\mathrm{l})$ triplexes at $25 \mathrm{mM} \mathrm{MgCl} 2$ salt concentration. 
CD Studies: $\quad$ CD spectroscopy has been shown to be a useful tool to distinguish between homopolymer A:T duplexes and T-A:T triplexes. We have used CD spectra here for three main purposes. First, to show that the A:T duplex and T-A:T triplex are present by comparison with previously published data. Second, for the spin labels to be useful for biological studies, it is important to demonstrate that they do not produce any significant conformational changes and thus give rise to $\mathrm{CD}$ spectra comparable with unmodified oligonucleotides. Moreover, since CD easily follows changes in secondary structure of nucleic acids ${ }^{31}$ with changes in solvent conditions, we conducted salt dependent CD measurements to show that the modification has a stabilizing effect on the triplex structure. Finally, CD data can be used to detect the presence of the triplex form, the duplex form, and the temperature dependent conversion of the former to the latter, thus the methodology can be used as an alternative or supplement to thermal denaturation data.

In Figure 3.9 are shown the $C D$ curves for $A_{15}: T_{15}$ and $A_{15}:{ }^{6 s p} T_{15}$ samples in $10 \mathrm{mM}$ phosphate buffer, $\mathrm{pH}$ 7.4, $100 \mathrm{mM}$ sodium chloride, and $50 \mathrm{mM}$ magnesium chloride over the temperature range from $30^{\circ} \mathrm{C}$ to $70^{\circ} \mathrm{C}$. Over all, the curves are quite similar and show characteristic positive ellipticity at $220 \mathrm{~nm}$ and $282 \mathrm{~nm}$ and negative ellipticity at $248 \mathrm{~nm}$ and $204 \mathrm{~nm}$.

As the temperature increases from $30^{\circ} \mathrm{C}$ to $75^{\circ} \mathrm{C}$, the positive intensity at $220 \mathrm{~nm}$ decreases gradually and stays at $220 \mathrm{~nm}$, while the intensity at 282 does not change and there is some noticeable shifting of $282 \mathrm{~nm}$ peak towards the higher wavelength. As for the negative ellipticity at $248 \mathrm{~nm}$, there is also a decrease in intensity with increasing temperature and very mild shift towards the higher wavelength. The overall shape of the CD curves and the observed minima and maxima for both modified and unmodified duplexes are 
a)

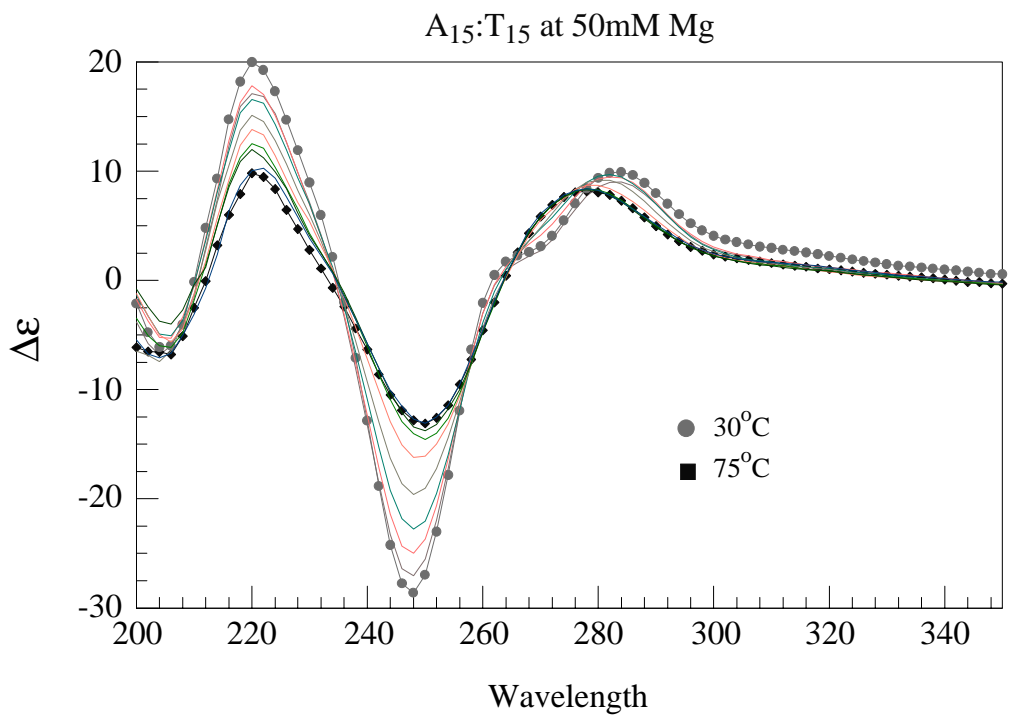

b)

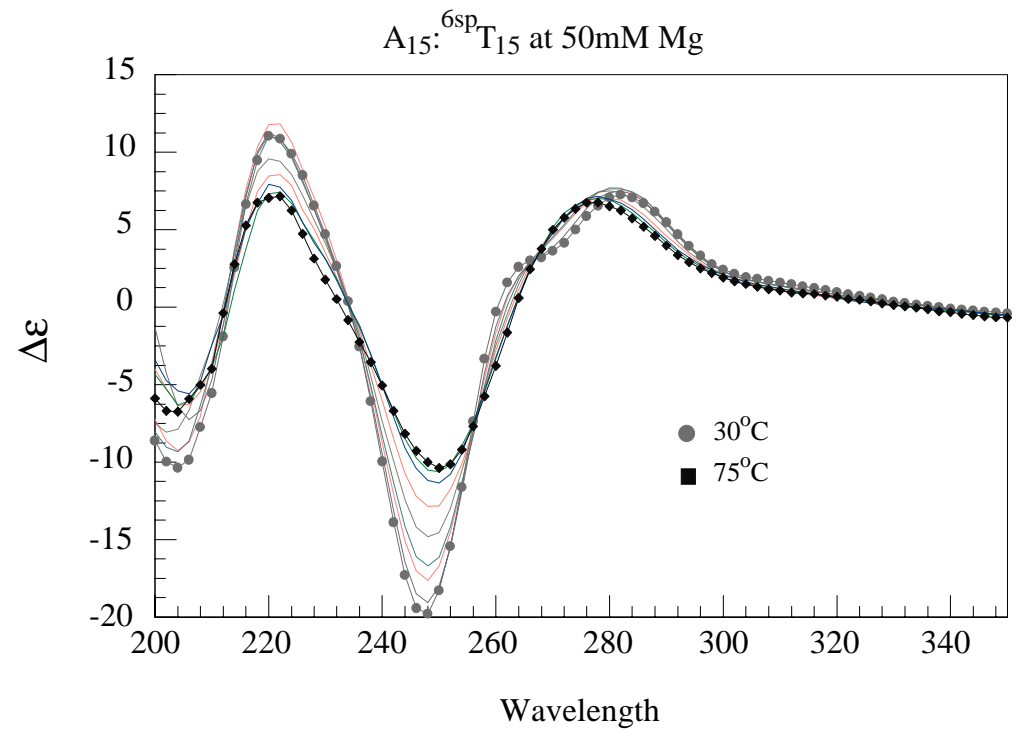

Figure 3.9. CD spectra as a function of temperature of (a) ${ }^{6 s p} \mathrm{~T}_{15}: \mathrm{A}_{15}$ duplex and (b) $\mathrm{A}_{15}: \mathrm{T}_{15}$ duplex recorded from $30^{\circ} \mathrm{C}(\bullet)$ to $75^{\circ} \mathrm{C}(\square)$. All samples were prepared in $10 \mathrm{mM}$ phoshpate buffer, $\mathrm{pH} 7.4,100$ $\mathrm{mM} \mathrm{NaCl}$ and $50 \mathrm{mM} \mathrm{MgCl}_{2}$. 
similar, except for are some noticeable differences. In particular, the positive ellipticities at 220 and $282 \mathrm{~nm}$ of spin labeled duplex and negative ellipticities at 204 and $248 \mathrm{~nm}$ are less intense, than those for unmodified ones. While the source of these differences is unknown, they may be due to differences in the light absorption properties of the spin label. However, the $\mathrm{CD}$ curves suggest that the conformation of the spin labeled duplexes is basically the same as the unlabeled duplex.

As it was mentioned before, CD spectra can be used to distinguish between triplex and duplex DNA for homopolymers of T-A:T and A:T, respectively. Here, we have measured the CD spectra of ${ }^{*} \mathrm{~T}_{15}-\mathrm{A}_{15}: \mathrm{T}_{15}$ samples ( ${ }^{*} \mathrm{~T}_{15}=\mathrm{T}_{15}$ and ${ }^{6 \mathrm{sp}} \mathrm{T}_{15}$ ) at different $\mathrm{MgCl}_{2}$ salt concentration. Figure 3.4a shows a series of CD spectra recorded on a sample containing $\mathrm{T}_{15^{-}}$ $\mathrm{A}_{15}: \mathrm{T}_{15}$ over a temperature range of $5^{\circ} \mathrm{C}-65^{\circ} \mathrm{C}$ at $10 \mathrm{mM} \mathrm{MgCl} 2$ concentration. The figure is divided into two parts for the better representation of $\mathrm{tx} \rightarrow \mathrm{ds}$ (I) and ds $\rightarrow$ coil (II) transitions. As one can notice from the temperature changing pattern of the Figure 3.10a (I), there is no indication of triplex formation at that particular salt concentration. Although, more carefully examination of the band at $5 \mathrm{C}$, indicates that there might be some triplex present, because of the appearance of the peak at $260 \mathrm{~nm}$ wavelength. The pattern of the changing intensities for both positive and negative bands with increasing temperature is similar to the one for the duplexes as shown in Figure 3.9. Though the shape of the CD curves is slightly different for the triplex compared to the duplex, the observed minima and maxima suggest that mainly duplex with some indication of triplex formation is observed. 

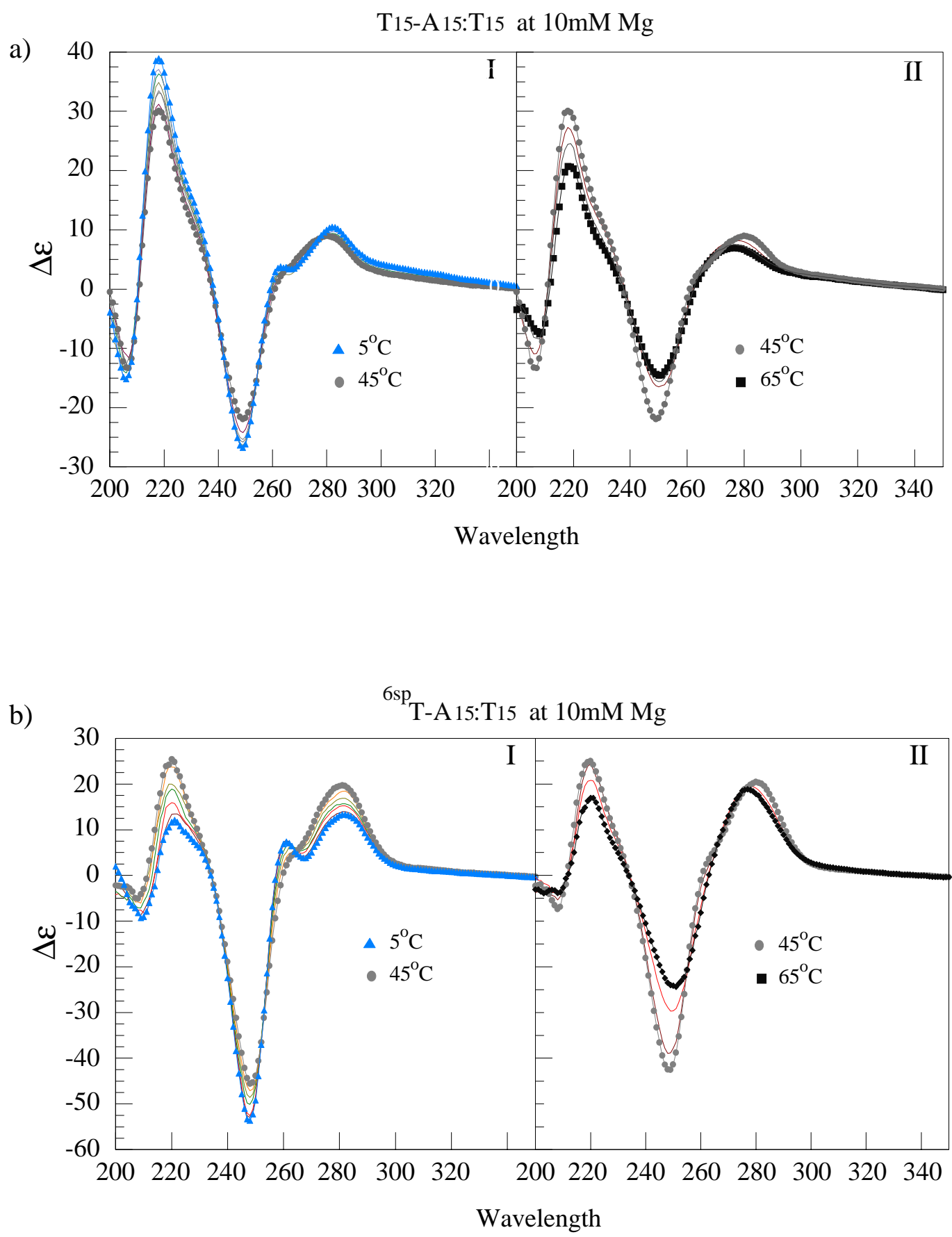

Figure 3.10. CD spectra as a function of temperature of a) $\mathrm{T}_{15}-\mathrm{A}_{15}: \mathrm{T}_{15}$ triplex and b) ${ }^{6 \mathrm{sp}} \mathrm{T}_{15}-\mathrm{A}_{15}: \mathrm{T}_{15}$ triplex recorded from $5^{\circ} \mathrm{C}(\Delta)$ to $45^{\circ} \mathrm{C}(\bullet)$ and from $45^{\circ} \mathrm{C}(\bullet)$ to $65^{\circ} \mathrm{C}(\boldsymbol{\nabla})$. All samples were prepared in $10 \mathrm{mM}$ phosphate buffer, $\mathrm{pH} 7.4,100 \mathrm{mM} \mathrm{NaCl}$ and $10 \mathrm{mM} \mathrm{MgCl}$ salt concentration. 
By looking at the Figure 3.10b we see a different pattern in CD spectra for the modified ${ }^{6 \mathrm{sp}} \mathrm{T}_{15}-\mathrm{A}_{15}: \mathrm{T}_{15}$ triplex at $10 \mathrm{mM} \mathrm{MgCl} 2$ salt concentration. The characteristic features to note in this figure are the following: at $5^{\circ} \mathrm{C}$ there are maxima at 220, 260, and 282 $\mathrm{nm}$, minima at 213, 248, and $267 \mathrm{~nm}$, and an isoelliptic point at $263 \mathrm{~nm}$. These spectral features are very similar to previously reported CD spectra of T-A:T triplexes and strongly support the presence of a triplex. Another important point to mention is that at low $\mathrm{MgCl}_{2}$ salt concentration, at which the presence of unmodified triplex is only weakly suggested, both acetylenic tether ${ }^{42}$ and the nitroxide have a stabilizing effect on triplex formation. As the sample is warmed from $5^{\circ} \mathrm{C}$ to $45^{\circ} \mathrm{C}$, the maximum at 220 and $282 \mathrm{~nm}$ increases with no change in the position of the peaks, while the maximum at $260 \mathrm{~nm}$ gradually diminishes in amplitude and slightly shifts towards the longer wavelength.

Further increase of the temperature from $45^{\circ} \mathrm{C}$ to $60^{\circ} \mathrm{C}$ a produce new set of spectral changes (Figure 3.10b II) similar to the CD of a duplex DNA. First, several of the characteristics of the spectrum at $45^{\circ} \mathrm{C}$, where the ds DNA form should be the main species present, are quite different from those observed at $5^{\circ} \mathrm{C}$. Here the intensity of a maximum at $220 \mathrm{~nm}$ decreases and the maximum at $260 \mathrm{~nm}$ is no longer present. However, the maximum at $282 \mathrm{~nm}$ and the minimum at $248 \mathrm{~nm}$ are still present though the former has increased in intensity and the latter has diminished. Raising the temperature produces smaller changes in the two maxima at $282 \mathrm{~nm}$ and $220 \mathrm{~nm}$, relative to those seen for the triplexes. In contrast, the relative change seen for the minimum at $248 \mathrm{~nm}$ is larger at higher temperatures for the duplex than were observed for the triplex. These observations, in total, are completely analogous to those reported for $\mathrm{T}_{12}-\mathrm{A}_{12}: \mathrm{T}_{12}$. 
By increasing the $\mathrm{MgCl}_{2}$ salt to $50 \mathrm{mM}$ concentration the $\mathrm{CD}$ spectra of both unmodified and spin-label modified triplexes reflect the characteristic features of $t x \rightarrow d s$ transition in the temperature range from $10^{\circ} \mathrm{C}$ to $45^{\circ} \mathrm{C}$. The representative $\mathrm{CD}$ spectra are shown in Figure 3.11. The spectra of the Figure 3.11a and 3.11b are quite similar to the spectra shown in Figure 3.10b. Upon heating the sample to $45^{\circ} \mathrm{C}$ the two characteristic maxima at 228 and $282 \mathrm{~nm}$ increase in the intensity while the maximum at $260 \mathrm{~nm}$ decreases. The only difference of these spectra is in short wavelength maxima at $228 \mathrm{~nm}$. It is significantly blue shifted $228 \rightarrow 218$ as opposed to being stationary in Figure 3.10b (I). Raising the temperature above $45^{\circ} \mathrm{C}$ produces $\mathrm{CD}$ spectrum that follows the pattern of the duplex. This suggests that ${ }^{6 \mathrm{sp}} \mathrm{T}$ spin label does not significantly affect the structure or conformation of the triplex it is part of. In fact, it may promote triplex formation at certain $\mathrm{MgCl}_{2}$ salt concentrations, not seen with unmodified triplexes. Likewise, the melting temperature curves of all sets of spectra are very similar and support the idea that the spin label modified thymidine is nearly identical with a thymidine which it replaces. Finally, the spectra shown here are consistent with those previously reported for unmodified T-A:T triplexes and A:T duplexes. 
a)

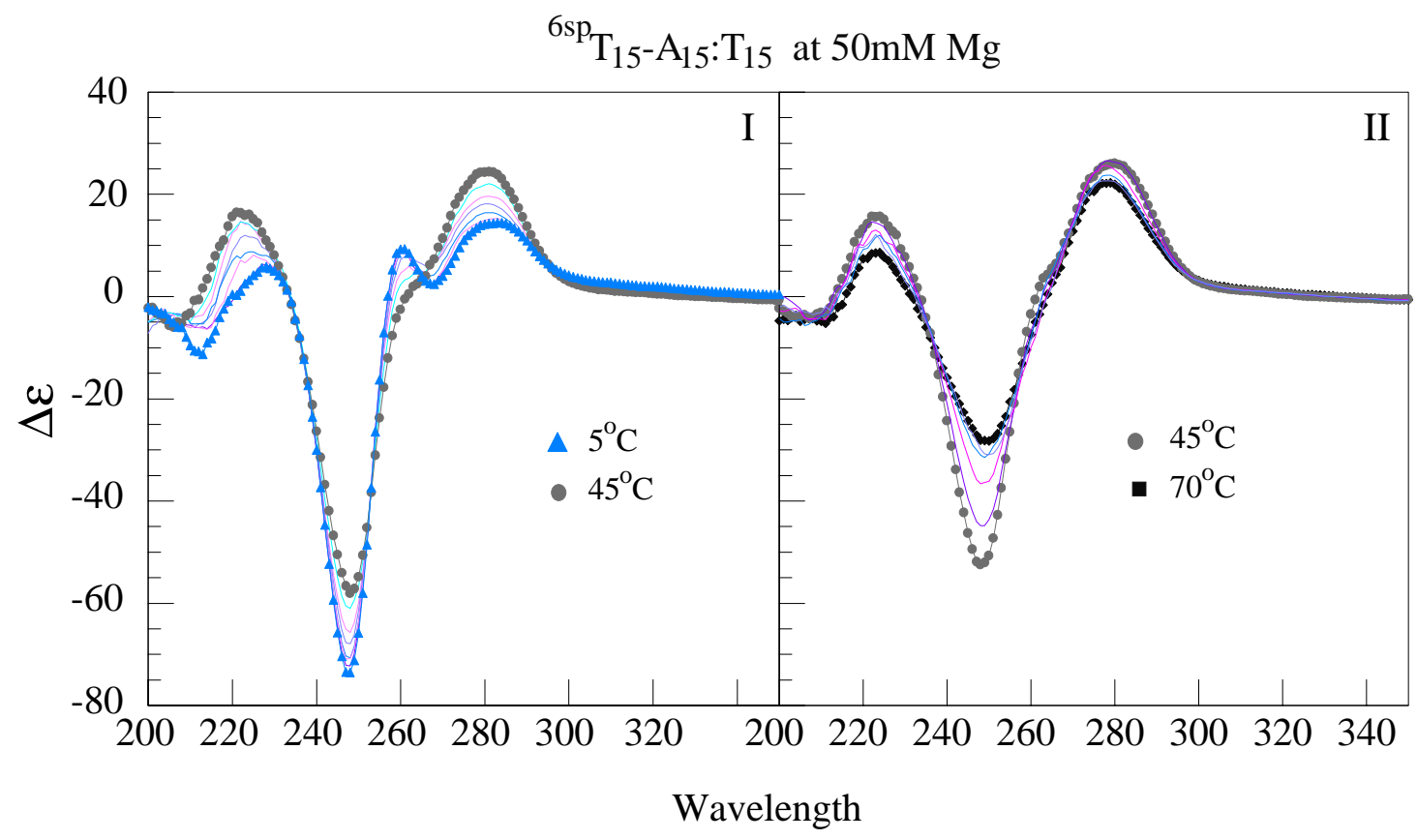

b)

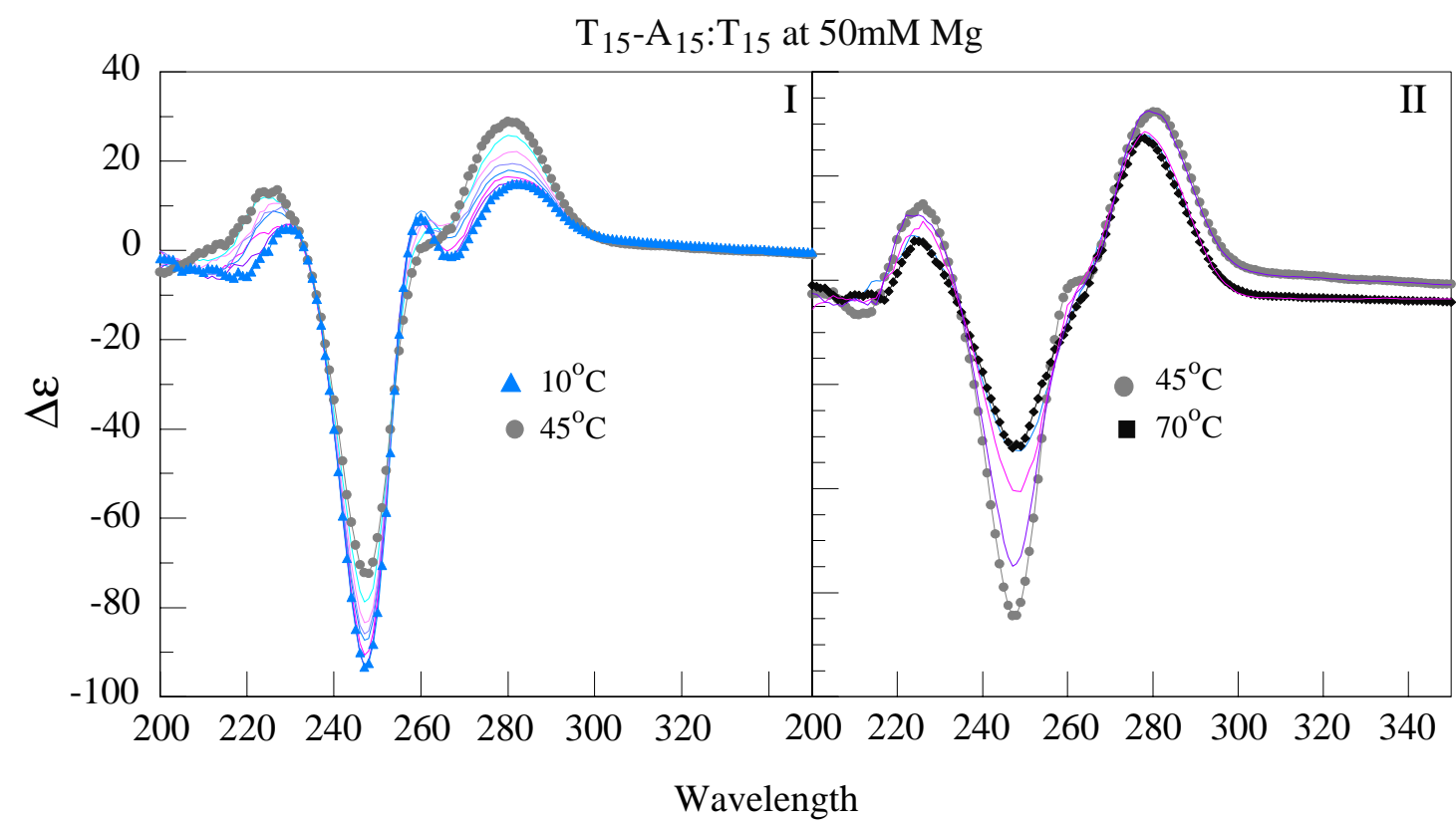

Figure 3.11. CD spectra as a function of temperature of a) $\mathrm{T}_{15}-\mathrm{A}_{15}: \mathrm{T}_{15}$ triplex and (b) ${ }^{6 \mathrm{sp}} \mathrm{T}_{15}-\mathrm{A}_{15}: \mathrm{T}_{15}$ triplex recorded from $5^{\circ} \mathrm{C}(\Delta)$ to $45^{\circ} \mathrm{C}(\bullet)$ and from $45^{\circ} \mathrm{C}(\bullet)$ to $70^{\circ} \mathrm{C}(\boldsymbol{)})$. All samples were prepared in $10 \mathrm{mM}$ phosphate buffer, $\mathrm{pH} 7.4,100 \mathrm{mM} \mathrm{NaCl}$ and $50 \mathrm{mM} \mathrm{MgCl}_{2}$. 
EPR studies: The EPR spectra of ${ }^{6 \mathrm{~s}} \mathrm{~T}_{15}$ are shown in Figure $3.12 \mathrm{a}$ and $3.12 \mathrm{~b}$. These spectra are taken at two different temperatures $0^{\circ} \mathrm{C}$ and $25^{\circ} \mathrm{C}$, respectively. Both spectra show slight anisotropic broadening of the spectral line at the higher field around 3380 Gauss. A similar spectrum, at $0^{\circ} \mathrm{C}$, was obtained by other researchers ${ }^{18}$ for the 12 -mer spin-labeled oligonucleotide.

a)

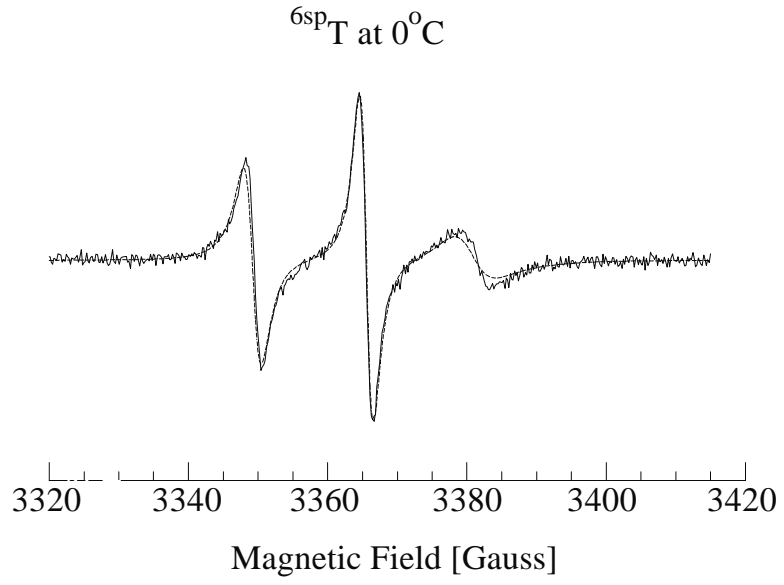

b)

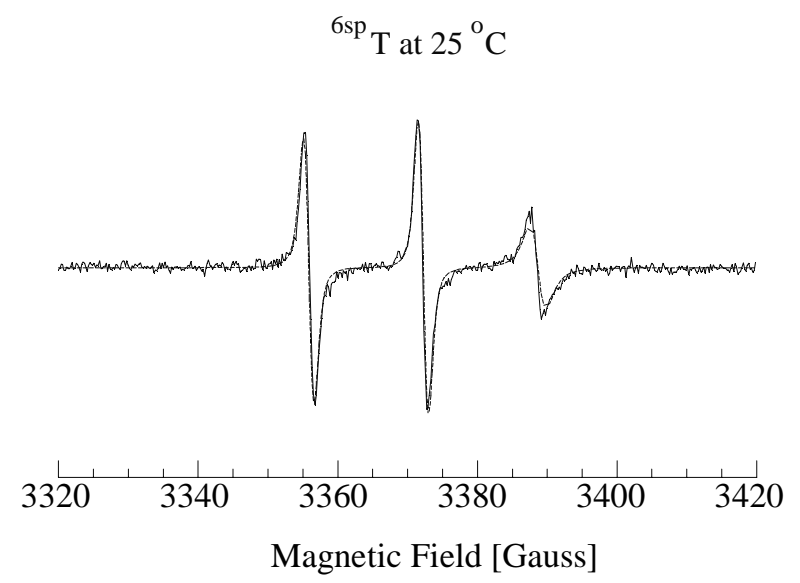

Figure 3.12. EPR spectra of ${ }^{6 s p} \mathrm{~T}_{15}$ at a) $0^{\circ} \mathrm{C}$ and b) $25^{\circ} \mathrm{C}$. The solid line is the experimental spectrum and the dashed line is the simulated spectrum.

The broadening of the spectral line indicates the degree of motional restriction of the spin-label. As the temperature gets colder and the motion becomes more restricted, resulting in more broadening. In order to extract correlation times $\left(\tau_{\mathrm{c}}\right)$ that would provide the degree of mobility for the spin label, these spectra were fitted using the program NLSL. ${ }^{15}$ The computer simulation fit is shown in this figure by dashed line. The fitting procedure provides the correlation times a measure of mobility for the spin label, with shorter times indicating greater mobility. 
The correlation time obtained for the spectrum at $0^{\circ} \mathrm{C}$ was approximately $3 \mathrm{~ns}$, slightly longer than the correlation time reported for the 12-mer (1 ns). The room temperature sample gives the correlation time approximately $1 \mathrm{~ns}$. The simulated values of correlation times at both $0^{\circ} \mathrm{C}$ and $25^{\circ} \mathrm{C}$ are summarized in Table 3.2

The EPR spectra of double stranded DNA sample $\mathrm{A}_{15} \cdot{ }^{6 \mathrm{sp}} \mathrm{T}_{15}$ were recorded at $0^{\circ} \mathrm{C}$ and $25^{\circ} \mathrm{C}$ and are illustrated in Figure 3.13a and 3.13b. Solid lines represent experimental spectra, while the dashed lines are the simulated ones. Looking at the spectra one can see that the

a)

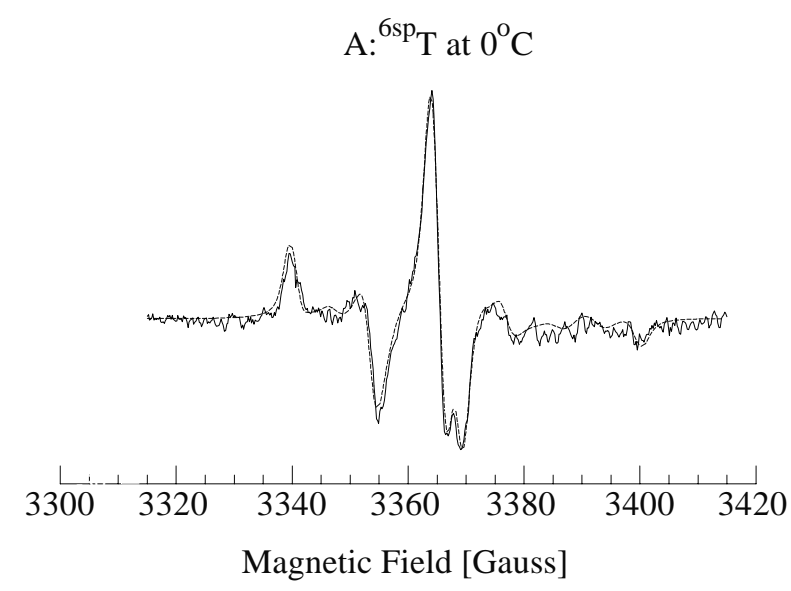

b)

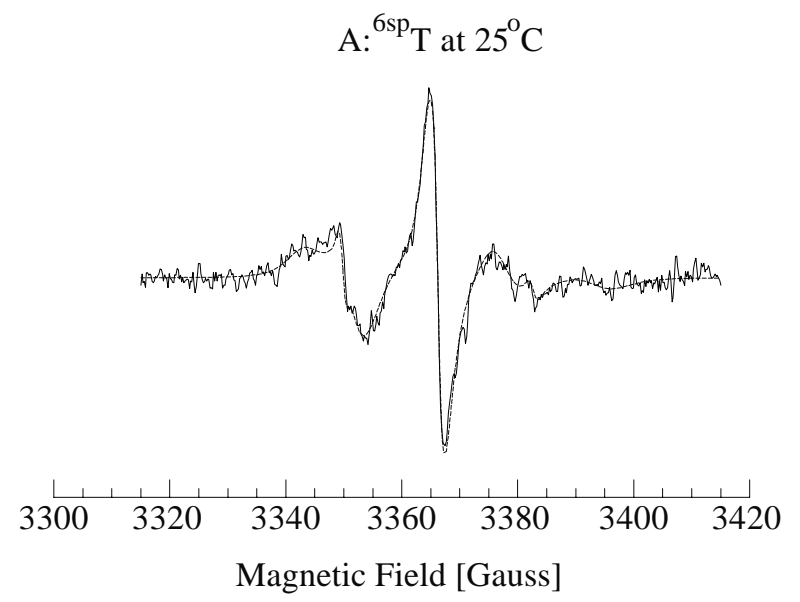

Figure 3.13. EPR spectra of $\mathrm{A}_{15}{ }^{6 \mathrm{sp}} \mathrm{T}_{15}$ at a) $0^{\circ} \mathrm{C}$ and b) $25^{\circ} \mathrm{C}$. The solid line is the experimental spectrum and the dashed line is the simulated spectrum.

mobility of spin label significantly reduced as a result of duplex formation. The spectra become very anisotropic as one can see by comparison of the EPR spectra in Figures 3.12 and 3.13. These spectra were simulated to calculate and average correlation, $\tau_{\mathfrak{c}}$, time is reported for both $0^{\circ} \mathrm{C}$ and $25^{\circ} \mathrm{C}$ temperatures. There are two possible ways of fitting the experimental data to extract correlation times. First, by assuming that there is only one structure present in 
the solution, which would correspond to a double stranded DNA. Second, assuming that there might be two species present in solution that could correspond to a duplex $\mathrm{A}_{15} \cdot{ }^{6 \mathrm{sp}} \mathrm{T}_{15}$, and some dissociated single strand ${ }^{6 \mathrm{sp}} \mathrm{T}_{15}$. The one species assumption produced correlation time of $163 \mathrm{~ns}$, but the overall fit was not very good.

The two species assumption would mean that the fit should result in two spectra with correlation times corresponding to one more mobile species (fast component) and the other more constraint (slow component). The spectra for the fast species have to resemble the spectra for the single stranded DNAs in the absence of $\mathrm{A}_{15}$ strand, and hence, the computed correlation times must be the same. Careful examination of the two spectra from simulation reveals that even though the overall fit is better than for one component assumption, the fast component spectra is far from being similar to single stranded DNA. The correlation times in Table 3.2 does not support this assumption either. Besides, this scenario is unlikely to be happening, especially at $0^{\circ} \mathrm{C}$, because of the stability of duplex at that temperature. Even at the room temperature the duplex stability is maintained, since its $\mathrm{T}_{\mathrm{m}}$ is still higher $\left(\sim 35^{\circ} \mathrm{C}\right)$ than room temperature, as shown from melting temperature studies. This is not the case as the fast species have correlation times roughly three times larger (Table 3.2). The results at room temperature are similar to those obtained at $0^{\circ} \mathrm{C}$. The one species fit gives a fast species that have shorter correlation times than the single stranded oligonucleotides.

As opposed to the single stranded oligonucleotides where we got similar correlation times as reported for ${ }^{5 \mathrm{sp}} \mathrm{T}_{12}$, the correlation times for duplexes we got are larger by a factor of 10 then were reported for $\mathrm{A}_{12}:{ }^{5 \mathrm{sp}} \mathrm{T}_{12}$. One could argue that this discrepancy could be the result of a use of different spin label. This is not the case, because the previous studies conducted in our lab on $5 \mathrm{sp}$ modified oligonucleotides produced similar spectra and correlation times for 
both ss and ds modified DNAs. On the other hand, the discrepancy may be due to the different methods used to fit the data. The correlation times for $\mathrm{A}_{12}:{ }^{5 \mathrm{sp}} \mathrm{T}_{12}$ were obtained by treating the duplex as a cylinder to which the probe was attached and analyzed using hydrodynamic theory. Here we have used the MOMD method for simulation. Which method is more appropriate for these systems is debatable. However, the basic conclusions are not affected.

\begin{tabular}{|c|c|c|c|c|c|c|c|}
\hline & ${ }^{6 s p} T$ & \multicolumn{3}{|c|}{${ }^{6 s p} T: A$} & \multicolumn{3}{|c|}{${ }^{6 s p} T-A: T$} \\
\hline & One & One & & & One & & \\
\hline & & & fast & slow & & fast & Slow \\
\hline $0^{\circ} \mathrm{C}$ & 3.03 & 167 & 10.5 & 157 & 170 & 4.83 & 307 \\
\hline $25^{\circ} \mathrm{C}$ & 1.07 & 10.7 & 0.33 & 25.7 & 0.95 & 0.60 & 18.3 \\
\hline$\tau_{\perp}$ & 4.056 & 38.57 & 62.89 & 109.5 & 53.40 & 30.78 & 38.73 \\
\hline$\tau_{\|}$ & 2.111 & 74.00 & 8.615 & 245.2 & 151.6 & 4.006 & 3570 \\
\hline $\mathrm{N} \%$ & 100 & 100 & 47 & 53 & 100 & 5 & 95 \\
\hline
\end{tabular}

${ }^{a}$ correlation times are expressed in nanoseconds (ns)

Table 3.2. Correlation times for single stranded, double stranded and triple stranded DNAs containing $\mathrm{T}^{6 \mathrm{sp}}$ spin labels. For two component fits, relative amounts in $\%$ of both species present are also listed.

As spectra become more anisotropic, meaning that the motion of the probe slows, it is more appropriate to calculate the two components of correlation time, $\tau_{\perp}$ and $\tau_{\|}$, since they are related to the rotational rate constants $\mathrm{R}_{\perp}$ and $\mathrm{R}_{\|}$. As the motion slows, the spectrum becomes more sensitive to $R_{\|}$and less sensitive to $R_{\perp}$. From these values more detailed information 
about anisotropy of the motion and the local ordering of the probe can be obtained. For that reason, we also reported calculated $\tau_{\perp}$ and $\tau_{\|}$at $0^{\circ} \mathrm{C}$ (Table 3.2).

As it was mentioned before, a variety of DNA structures such as double stranded DNA, loops, base pair mis-matches and bulges have been studied with $5 \mathrm{sp}$ nitroxide spin labels. But, so far their application did not go beyond these types of structures, moreover triplex DNAs were not investigated. We are showing here that the newly synthesized 6sp nitroxide spin label can also be used for detecting and monitoring triplex DNA formation, and it is behaving no different than its 5-membered counterpart.

It is important to mention the order of mixing oligonucleotides for triplex preparation, especially the spin labeled one. As it was described in the experimental section first, the duplex between $A_{15}$ and $T_{15}$ was prepared $\left(A_{15}: T_{15}\right)$. Then, to this duplex either $T_{15}$ or ${ }^{6 s p} T_{15}$ strands were added and samples were annealed overnight at $4^{\circ} \mathrm{C}$, under conditions known to produce tx DNA $\left(\mathrm{T}_{15}-\mathrm{A}_{15}: \mathrm{T}_{15}\right.$ or $\left.{ }^{6 \mathrm{sp}} \mathrm{T}_{15}-\mathrm{A}_{15}: \mathrm{T}_{15}\right)$. The EPR were recorded at both $0^{\circ} \mathrm{C}$ and at room temperatures. The resulting EPR and spectral simulations are shown in Figure 3.14a and 3.14b. It obvious that the spectra obtained at $0^{\circ} \mathrm{C}$ are anisotropic and the mobility of the strand has been constrained. This, in turn, implies that the third strand has become bound to its target and thus the EPR spectra shown in Figure 3.14a are those of triplex DNA. In addition, thermal denaturation and circular dichroism data also support this result.

The triplex EPR data were fit as described for the duplexes, fitting the data assuming either one or two species to be present. As in the case of the duplexes, the fits were improved for the two species fits and the calculated correlation times are shown in Table 3.2. However, unlike the duplexes, the correlation times obtained for the fast species agree with those obtained for the single stranded oligonucleotides. This, in turn, suggests that, in the case of a 
triplex, the two species fit is a better choice. Moreover, if the spectrum of the triplex ${ }^{6 \mathrm{sp}} \mathrm{T}_{15^{-}}$ $\mathrm{A}_{15}: \mathrm{T}_{15}$ is divided into two sub-spectra, as it is shown in Figure 3.14a, we can clearly see the presence of the fast and the slow species. The calculated spectrum of the fast species resembles the one used to fit $\mathrm{ss}^{6 \mathrm{sp}} \mathrm{T}_{15}$ at $0^{\circ} \mathrm{C}$ (Figure 3.12a) and the calculated correlation time also supports our assumption.

Correlation times that are obtained by simulating triplex spectra using the two site assumption, give values that are twice of those for the duplexes, which in turn was simulated assuming single species. This increase in correlation time suggests that triplex DNA may be more rigid that double stranded DNA. On the other hand, the difference may simply be due to the increased size of the triplex relative to the duplex. This would indicate that the spectra are influenced more by the global tumbling of the DNA molecule rather than by the local motion of the spin label. It is difficult to distinguish between these two possibilities and, particularly, to estimate the contribution of the local spin label motion to the overall EPR spectra. To be able determine which factor is responsible for the difference one will require additional studies, such as EPR studies at higher frequencies that will freeze out the global DNA tumbling and be more sensitive to faster molecular motion. In addition, molecular modeling and molecular dynamics calculations may further help to resolve this question, which were investigated in the following chapters.

One other question can arise from looking at the spectrum of the triplex DNA whether it represents triplex or duplex DNA. For that purpose, the temperature dependence of the triplex EPR data address whether the spectrum in Figure 3.14 is triplex DNA with the spin labeled strand as the Hoogsteen or the Watson-Crick base paired strand. As the temperature 

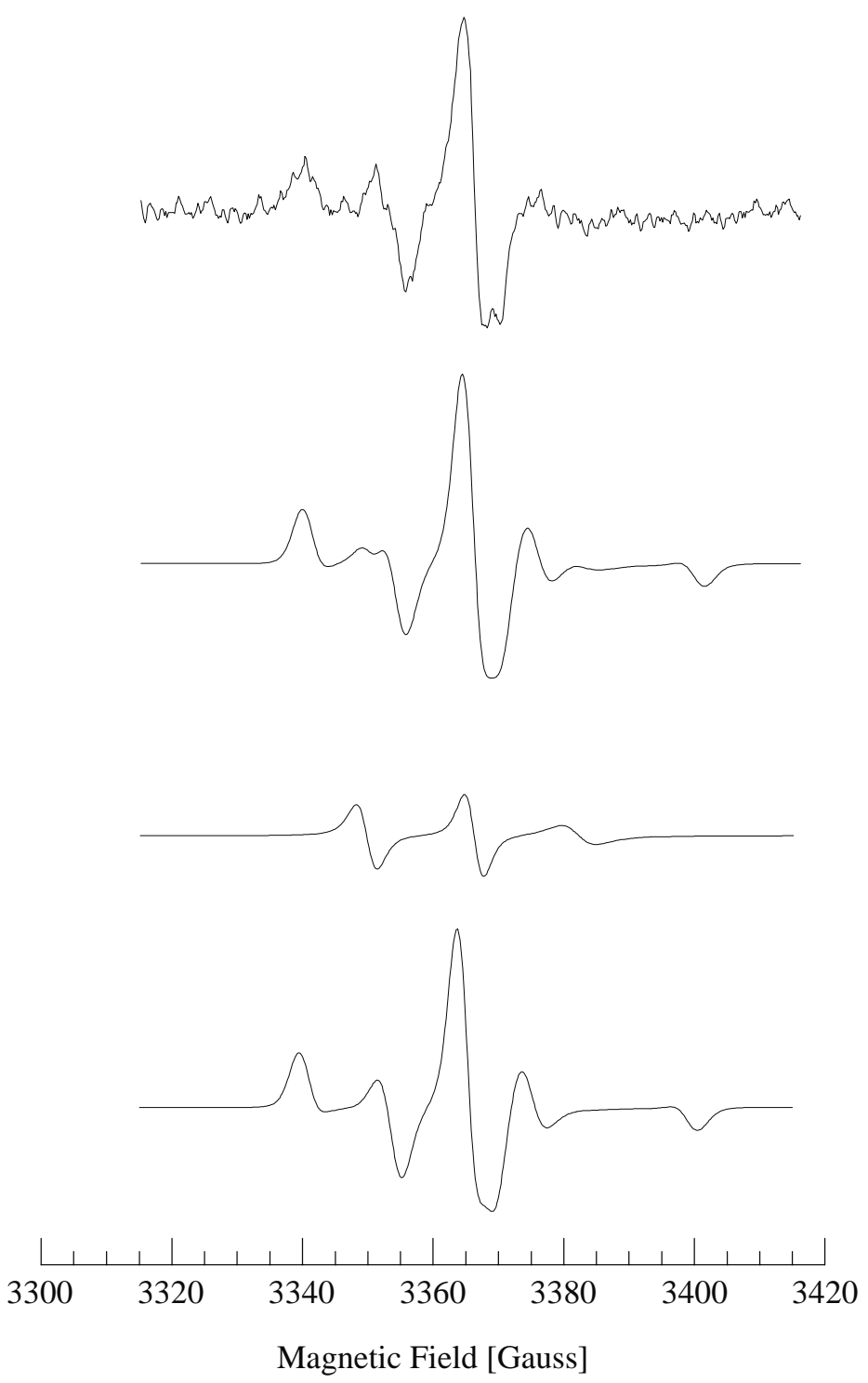

\section{Experimental Spectrum}

Simulated Spectra

Both components

Fast component

Slow component

Figure 3.14a. EPR spectra of: ${ }^{6 \mathrm{sp}} \mathrm{T}_{15}-\mathrm{A}_{15}: \mathrm{T}_{15}$ at $0^{\circ} \mathrm{C}$. Experimental and simulated spectra for both components as well as deconvoluted into fast and slow components are shown. 


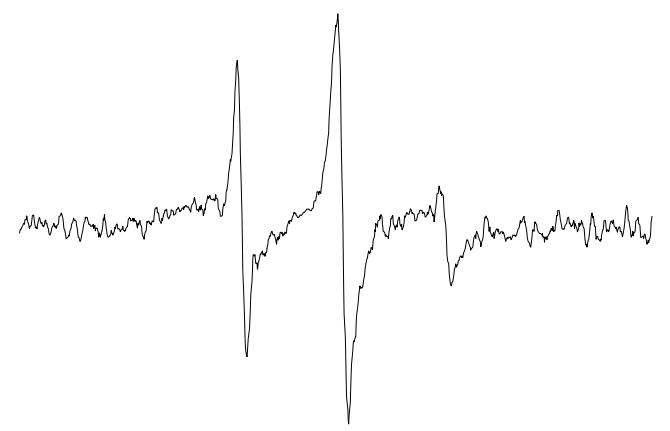

Experimental Spectrum

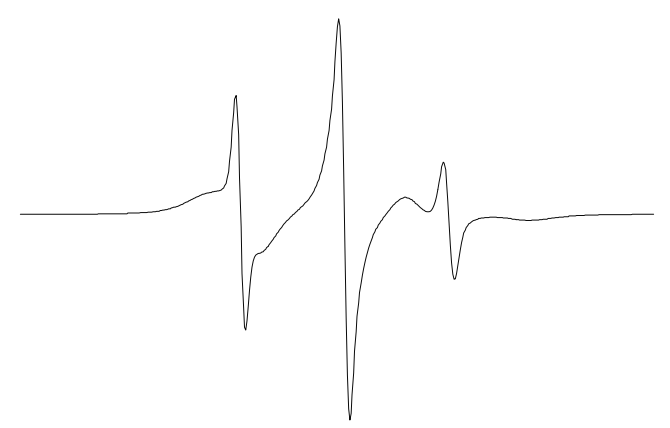

Simulated Spectra

Both components

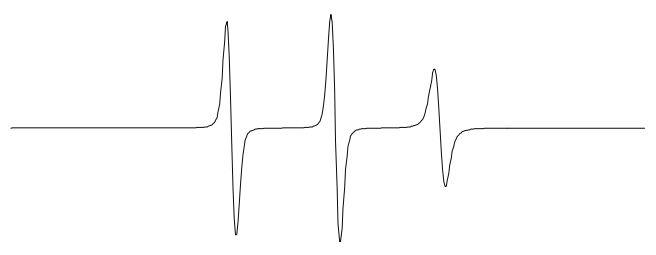

Fast component

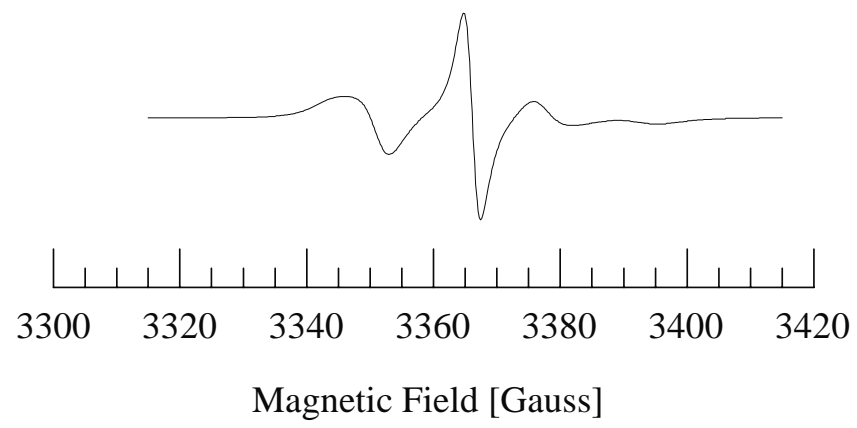

Slow component

Figure 3.14b. EPR spectra of: ${ }^{6 \mathrm{sp}} \mathrm{T}_{15}-\mathrm{A}_{15}: \mathrm{T}_{15}$ at $25^{\circ} \mathrm{C}$. Experimental and simulated spectra for both components as well as deconvoluted into fast and slow components are shown. 
is increased the complementary third strand starts to unwind and one can argue that the ${ }^{6 s p} \mathrm{~T}_{15}$ strand could have exchanged with the $T_{15}$. Thus the EPR spectrum shown in Figure 3.14a might correspond to $\mathrm{T}_{15}-\mathrm{A}_{15}:{ }^{6 \mathrm{sp}} \mathrm{T}_{15}$ instead of ${ }^{6 \mathrm{sp}} \mathrm{T}_{15}-\mathrm{A}_{15}: \mathrm{T}_{15}$. This seems unlikely as samples were maintained at room temperature or below, conditions where the duplex $\left(\mathrm{A}_{15}: \mathrm{T}_{15}\right)$ is stable $\left(\mathrm{T}_{\mathrm{m}} \sim 45^{\circ} \mathrm{C}\right)$. However, if the spin labeled strand had exchanged with the Watson-Crick strand, then the room temperature spectrum of the triplex (Figure 3.14b) should have looked like the spectrum of the duplex at $25^{\circ} \mathrm{C}$ (Figure 3.13b), which did not occur. It rather resembles the spectrum of ss DNA at $25^{\circ} \mathrm{C}$ (Figure 3.12b), but with lower resolution. The following conclusion can be drown, that the only species present in solution should be ds DNA with Watson-Crick base pairing $\left(\mathrm{T}_{15}: \mathrm{A}_{15}\right)$ and ss DNA $\left({ }^{6 \mathrm{sp}} \mathrm{T}_{15}\right)$. And the EPR spectrum that is detected is that of the ss DNA, which is actually a case (Figure 3.14b). 


\section{CHAPTER 4}

\section{Theoretical Background}

The structural and dynamical nature of biological molecules has been studied for several decades. The extent of their flexibility became more evident due to the availability of experimentally determined structures of these molecules in different environments. Even though experimental studies can reveal many aspects associated with conformational transitions, they are often unable to reveal the structural or conformational details of the

pathway between the end points. Such information can be readily addressed via computational methods.

Computational approaches have been used to investigate the energies associated with changes in both conformation and chemical structure, to facilitate the experimental macromolecular structure determination, and structural refinement based on their NMR or Xray data. Recent advances in techniques that combine quantum mechanical (QM) and molecular mechanics (MM) approaches, allow us to understand the microscopic processes controlling reaction rates, and processes involved in creating and breaking bonds. In this chapter the computational techniques used in the current work to model unmodified and spin label modified nucleic acids are outlined.

\subsection{Quantum Mechanics (QM)}

Nitroxides are one of the few classes of organic free radicals that are stable under ordinary conditions; they are isolable compounds and can be stored indefinitely. This has allowed the determination of a number of molecular structures by using x-ray crystallographic methods and the consequent unequivocal correlation between structural and spectroscopic 
parameters. This characteristic, in turn, coupled to the strong localization of the unpaired spin in the NO moiety, makes nitroxides ideal "Spin Labels" useful for exploring the structure of short-lived free radicals by Electron Spin Resonance (ESR) spectroscopy.

The use of spin-labeling to study some characteristics of nucleic acids such as their base dynamics, their interactions with complementary nucleic acids, and their interactions with nucleic acid binding proteins and drugs has been well documented in recent years. Until recently available force fields lacked suitable parameters for nitroxide species. Barone et al., studied the structures and spectromagnetic properties of some model nitroxides by selfconsistent hybrid of Hartree-Fock and density functional methods (B3LYP) obtaining results close to experimental data. From the computed structures, together with the available experimental data, new parameters for the NO moiety have been developed for two of the most commonly used force fields MM3 and MM+. These force fields are applicable for small molecules, with the maximum number of atoms limited to 500, but they provided the background for computations of reliable structures of large molecules like proteins and nucleic acids, containing nitroxide systems.

The quantum mechanics calculation program Q-Chem, where the quantum-mechanical calculations are based on unrestricted Kohn-Sham approach to density functional theory (DFT), was used in this work. Density functional methods following the Kohn-Sham formalism are based on parameter-free theory, i.e., they attempt to find solutions "from first principles" to the self-consistent functional (SCF) mean-field model of the electronic structure, while treating the electron correlation problem differently than the post-HartreeFock techniques. The basic idea of the DFT is to use the electron density $\rho(\boldsymbol{r})$ as the variable 
of the system instead of electronic wavefunction. The total energy of an n-electron system an be expressed as

$$
E=E_{T}+E_{V}+E_{J}+E_{X C}
$$

$E_{T} \quad$ is the kinetic energy of noninteracting electrons with the same total electron density as the actual system of interacting electrons,

$E_{V} \quad$ is electron-nuclear interaction energy,

$E_{J} \quad$ is the Coulomb self-interaction of the electron with density $\rho(\boldsymbol{r})$ and

$E_{X C} \quad$ is the energy of exchange interactions, correlation effects, and the difference between exact kinetic energy and that of the reference system of noninteracting electrons with the density $\rho(\boldsymbol{r})$. Adopting an unrestricted format, the $\alpha$ and $\beta$ total electron densities can be written as

$$
\begin{aligned}
& \rho_{\alpha}(\boldsymbol{r})=\sum_{i=1}^{n_{\alpha}}\left|\Psi_{i}^{\alpha}\right|^{2} \quad \mathbf{2 . 2} \quad \text { and } \quad \rho_{\beta}(\boldsymbol{r})=\sum_{i=1}^{n_{\beta}}\left|\Psi_{i}^{\beta}\right|^{2} \\
& \rho(\boldsymbol{r})=\rho_{\alpha}(\boldsymbol{r})+\rho_{\beta}(\boldsymbol{r})
\end{aligned}
$$

The components of Equation 2.1 can now be written as

$$
\begin{aligned}
E(\rho)= & -\frac{1}{2} \sum_{i} \int \Psi_{i}^{*}\left(\boldsymbol{r}_{1}\right) \nabla_{1}^{2} \Psi_{i}\left(\boldsymbol{r}_{1}\right) d \boldsymbol{r}_{1}+\sum_{A} \int \frac{Z_{A}}{\left|\boldsymbol{r}_{1}-\boldsymbol{R}_{A}\right|} \rho\left(\boldsymbol{r}_{1}\right) d \boldsymbol{r}_{1} \\
& +\frac{1}{2} \iint \frac{\rho\left(\boldsymbol{r}_{\boldsymbol{I}}\right) \rho\left(\boldsymbol{r}_{2}\right)}{\left|\boldsymbol{r}_{\boldsymbol{I}}-\boldsymbol{r}_{2}\right|} d r_{1} d r_{2}+E_{X C}(\rho)
\end{aligned}
$$

Although innocuous in appearance, $E_{X C}$ contains, buried within it, all the details of two-body exchange and dynamical correlation and a kinetic energy component as well and requires a more in depth discussion. The B3LYP hybrid functional, which combines HartreeFock and Becke exchange terms with Lee-Yang-Parr correlation functional, with the standard 6-31 $\mathrm{G}^{*}$ basis set have been used for geometry optimization of a training set of three small 
nitroxide molecules $\mathrm{MNO}$, TBNO and 5-membered ring nitroxide radical. Geometry optimization refers to the determination of stationary points, principally minima and transition states on molecular potential energy surfaces. It is an interactive process, requiring the repeated calculation of energies, gradients at each optimization cycle until convergence is achieved. The form of the B3LYP functional is given in Equation 4.5.

$E_{x c}^{B 3 L Y P}=\left(1-a_{0}\right) E_{x}^{L S D A}+a_{0} E_{x}^{H F}+a_{x} \Delta E_{x}^{B 88}+a_{c} E_{c}^{L Y P}+\left(1-a_{c}\right) E_{c}^{V W N}$

where $\Delta \mathrm{E}_{\mathrm{x}}{ }^{\mathrm{LSDA}}$ is local spin-density approximation, which uses the standard local exchange functional and local correlation functional of Vosko, Wilk and Nusair $\Delta \mathrm{E}_{\mathrm{c}}{ }^{\mathrm{VWN}}$.

$\Delta \mathrm{E}_{\mathrm{x}}{ }^{\mathrm{HF}}$ is exact Hartree-Fock exchange

$\Delta \mathrm{E}_{\mathrm{x}}{ }^{\mathrm{B} 88}$ is Becke's gradient correlation to the exchange functional

$\Delta \mathrm{E}_{\mathrm{c}}^{\mathrm{LYP}}$ is Lee-Yang-Parr correlation functional

Becke suggested coefficients $a_{0}=0.2, a_{x}=0.72, a_{c}=0.81$ based on fitting to heats of formation of small molecules. Final geometry output is used as input for Gaussian94 single point calculation in order to get electrostatic points in a form, suitable for use in restrained electrostatic potential fit model RESP. RESP will fit the quantum chemically calculated potential at molecular surface using an atom-centered point charge model. 


\subsection{Molecular Mechanics (MM)}

The applications of molecular mechanics (MM) have employed energy minimization, molecular dynamics, and Monte Carlo methods to move on the analytical potential energy surfaces. They are capable of giving insight into the entire spectrum of non-covalent interactions between molecules, and, when combined with quantum mechanical electronic structure calculations, which model covalent bonding changes, essentially all molecular reactions and interactions can be examined. Given their importance, much effort has gone into establishment of both the functional form of equations and the parameters required for applying such analytical energy functions or, otherwise, called "force fields".

Molecular mechanical force fields are based on the concept that the potential surfaces and physical properties of molecules may be calculated using classical mechanics functions. A potential energy function is a mathematical equation that allows for the potential energy, $U$, of a chemical system to be calculated as a function of its three-dimensional (3D) structure, $r$. A molecule is thought of as a collection of atoms, which interact with each other in a way that can be described by simple potential energy harmonic functions or force fields. AMBER is based upon a valence force field, and the total potential energy potential, $U_{t o t}$, which well describes interactions between atoms for large molecules, of a molecule, is given in Eq. 4.6.

$$
\begin{aligned}
U_{\text {int ernal }} & =\sum_{\text {bonds }} K_{r}\left(r-r_{0}\right)^{2}+\sum_{\text {angles }} K_{\theta}\left(\theta-\theta_{0}\right)^{2}+\sum_{\text {dihedrals }} \sum_{n} \frac{V_{n}}{2}[1+\cos (n \varphi-\gamma)] \\
& +\frac{1}{V D W_{\text {scale }}} \sum_{j=1}^{\text {bondsbonds }} \sum_{i>j}^{*} \varepsilon^{*}\left[\left(\frac{R_{i j}^{*}}{r_{i j}}\right)^{12}-\left(\frac{R_{i j}^{*}}{r_{i j}}\right)^{6}\right]+\frac{1}{E E L_{\text {scale }}} \sum_{j=1}^{\text {atomsatoms }} \sum_{i>j} \frac{q_{i} q_{j}}{r_{i j} \varepsilon}
\end{aligned}
$$


The first three terms that describe bond, angle, and dihedral potentials may be thought of as bonding interactions. The bond-stretching and angle-bending terms are treated harmonically, which keeps the bonds and angles near their equilibrium values $r_{0}$, and $\theta_{0} . K_{r}$ and $K_{\theta}$ are bond stretching and angle bending constants associated with the bond and angle terms. The dihedral term includes the force constant $V_{n}$ that dictates the magnitude of torsional barrier, the periodicity $n$ that indicates number of cycles per $360^{\circ}$, and a phase $\gamma$ that dictates the location of the maxima in the dihedral energy surface. Improper torsional angles, in which the atoms are not sequentially bonded to each other, use the same potential function as torsional angles. Improper dihedral angles are used to preserve planarity of specific ring fragments.

The next two terms, the Van der Waals interactions represented by a Lennar-Jones 612 potential and electrostatic potentials modeled by a Coulombic interactions of atomcentered point charges, describe the non-bonded interactions. These terms are considered the most important energy terms because of the strong influence of the environment on the properties of macromolecules as well as the large number of nonbond interactions occurring in molecules themselves. Parameters associated with these terms are van der Waals well depth for given atom, $\varepsilon_{i j}{ }^{*}=\left(\varepsilon_{\mathrm{ii}}{ }^{*} \varepsilon_{\mathrm{jj}}{ }^{*}\right)^{1 / 2}$, the minimum interaction radius, $R_{i j}{ }^{*}=\left(R_{i i} * R_{j j}{ }^{*}\right)^{1 / 2}$, dielectric constant, $\varepsilon$, and the partial atomic charges $q_{j}$. Electrostatic and van der Waals interactions are only calculated between atoms in different molecules or for atoms in the same molecule separated by at least three bonds. Those non-bonded interactions separated by exactly three bonds, "1-4 interactions", are reduced by the application of a scale factor. 


\subsection{Molecular Dynamics (MD)}

Molecular Dynamics is a method that can be used for studying the motions and the conformational space of molecular systems or as a tool to study the structure of molecular systems. Given a potential energy function and its associated force field the classical Newtonian equations of motion can be numerically integrated for all the atoms in the system. In order to start a simulation velocities, $v_{i}$ are assigned to all $\mathrm{N}$ particles with mass $m_{i}$ from Boltzmann distribution about given temperature, $\mathrm{T}$.

$$
\frac{1}{2} \sum_{i}^{N} m_{i} v_{i}^{2}=\frac{3 N k T}{2}
$$

The positions and velocities that a particle will have in future are calculated by first determining the force on each particle, $\boldsymbol{F}_{\boldsymbol{i}}$ as a function of time, and acceleration, $\boldsymbol{a}_{\boldsymbol{i}}$ as shown below:

$$
\boldsymbol{a}_{i}=\frac{\boldsymbol{F}_{i}}{m_{i}}=-\frac{1}{m_{i}} \frac{\partial U}{\partial \boldsymbol{r}_{i}}
$$

The Hamiltonian of the system in terms of kinetic and potential energy is given in Equation 4.9, where $U(\boldsymbol{q})$ has the form of the AMBER energy function (Equation 4.6).

$$
H(\boldsymbol{q}, \boldsymbol{p})=\frac{1}{2} \sum_{i=1}^{N} \frac{\boldsymbol{p}_{i}^{2}}{m_{i}}+U(\boldsymbol{q})
$$

The velocity Verlet ${ }^{43}{ }^{44}$, is one of a variety of first order integration algorithms that is typically used to integrate the equations of motion, to obtain the coordinates for the next step:

$$
\boldsymbol{q}_{i}(t+\delta t)=\boldsymbol{q}_{i}(t)+\delta t v_{i}(t)+\frac{\delta t^{2}}{2 m_{i}} \boldsymbol{F}_{i}(t)
$$

This technique involves updating the velocities in two steps. In the first step, the velocities

are advanced from time t to time $\left(t+\frac{\delta t}{2}\right)$. Then forces $\boldsymbol{F}$ are updated for the new atomic 
positions and the velocities are updated from time $\left(t+\frac{\delta t}{2}\right)$ to time $(t+\delta t)$ and are calculated by:

$$
\boldsymbol{v}_{i}(t+\delta t)=\boldsymbol{v}_{i}(t)+\frac{\delta t}{2 m_{i}}\left[\boldsymbol{F}_{i}(t+\delta t)+\boldsymbol{F}_{i}(t)\right]
$$

The resulting set of positions and velocities, $\boldsymbol{R}=\left\{\boldsymbol{q}\left(t_{1}\right), \boldsymbol{q}\left(t_{2}\right) \ldots \boldsymbol{v}\left(t_{1}\right), \boldsymbol{v}\left(t_{2}\right)\right\}$, is referred to as the dynamics trajectory. Thus, with this method both the positions and velocities are known at the same time step, which has several advantages over other algorithms. One important point is that the time step can be altered without greatly affecting the system. The second point is that several methods for coupling to temperature or pressure baths can be utilized.

DNAs are highly charged molecules and their representation with simple potential present special difficulties. Water is an important and integral part of any nucleic acid, so the explicit introduction of the solvent becomes fairly critical. It plays a structural role acting both as H-bond donor or acceptor, and it has polarization effects by screening the electrostatic interactions due to its high dielectric constant ( 80). However, the high charge density from phosphate backbone and profound salt dependence of nucleic acids secondary structure require explicit inclusion of counterions to cover for ionic screening. Even with explicit representation of water and ions, the complexity of the calculation centers on the proper evaluation of the pairwise non-bonded and Coulombic interactions.

Many simulations with explicit solvent have been performed on DNA molecules. They were limited to a short time scale ( 100ps) and typically displayed anomalous structure ${ }^{45}$, such as base fraying, which demonstrated the necessity for inclusion of more accurate representation of solvent. More recent simulations on longer $\sim 1 \mathrm{~ns}$ time scale suggest the importance of properly treating the long-ranged electrostatic interactions ${ }^{46},{ }^{47}$. The 
application of the particle mesh Ewald method allows simulations of nucleic acids with explicit solvent and counterions in a nanosecond time range. In the particle mesh Ewald (PME) method, a Gaussian charge distribution of opposite sign is superimposed upon the original point charge, producing a screened charge distribution. The electrostatic interaction between the screened charges is short-ranged. The original distribution is recovered by adding a second Gaussian charge distribution identical to the first, but opposite sign. The interaction between these canceling distributions is calculated in reciprocal or k-space.

$$
E_{\text {recip }}=\frac{1}{2 \pi V} \sum_{m \neq 0} \frac{e^{-\pi^{2} m^{2} / k^{2}}}{m^{2}}\left|S(m)^{2}\right|
$$

where $S(m)=\sum_{i=1} q_{i} e^{2 \pi i m \cdot r_{i}}, \mathrm{~V}$ denotes the volume and $\mathrm{m}$ the reciprocal vectors

MD can be performed in different ensembles such as NVE, where evolution of classical system of $N$ particles in a volume $V$ is considered. Here, the total energy $E$ is the constant of motion. There are also more scientifically relevant ensembles, like canonical NVT, associated with Helmholtz free energy or isothermal-isobaric NPT ensemble associated with a Gibbs free energy. These are used in current MD methodology.

During constant temperature molecular dynamics, the velocities, $v_{i}$, in the system are scaled during each time step of MD. This couples the system, with a temperature relaxation time $\tau_{T}$, to a heat bath at $T_{0}$. Velocities are scaled by a factor $\lambda v$, which allows maintaining a desired amount of kinetic energy, where

$$
\lambda=1+\frac{\delta t}{2 \tau_{T}}\left(\frac{T_{0}}{T-1}\right)
$$


where $T$ is instantaneous temperature. In a manner similar to the velocity scaling, the pressure is held constant by scaling the atomic coordinates by a factor $\mu$, so that a desired pressure is maintained:

$$
\mu=\left[1-\frac{\delta t}{\tau_{P}}\left(P_{0}-P\right)\right]^{\frac{1}{3}},
$$

where $P$ is instantaneous pressure. 


\section{CHAPTER 5}

\section{Theoretical Procedures}

Molecular Dynamics (MD) can be used to produce high-resolution trajectories through the confined region of the phase space. It can provide detailed information of the dynamic behavior of different DNA structures over the 1-5 ns trajectory range. Molecular dynamics can be applied to molecules of biological importance and specifically to nucleic acids and DNAs with modifications. These modifications can drive conformational changes, have an impact on structure stability, or serve as probes to sample conformational space of biomolecules.

In order to be able to conduct MD simulations with modified DNAs, it is necessary to incorporate appropriate parameters into the force fields. These parameters can come from either known experimental data, such as crystallographic (X-ray) or spectroscopic (IR, NMR) or from ab initio quantum mechanical calculations if no experimental data have been or can be obtained.

The current chapter will summarize the methodology applied to developing suitable parameters for $5 \mathrm{sp}$ and $6 \mathrm{sp}$ nitroxides using model compounds. Validation of these parameters for $5 \mathrm{sp}$ and $6 \mathrm{sp}$ nitroxides then follows, with modeling of unmodified and spinlabel modified ss, ds, and tx DNAs. 


\subsection{Methodology}

\subsubsection{Development of nitroxide Force Field Parameters}

In order to run simulations with spin labeled modified DNAs suitable parameters for 5sp and 6sp molecules had to be developed and incorporated into the Cornell et al. 1994 force field of the Amber 5.0 suite. As a starting point we have chosen two model compounds, MNO and TBNO, whose structures are shown in Figure 5.1. These nitroxide compounds have experimental data to compare our results with. They have also been parameterized in MM+ and UFF molecular mechanics force fields ${ }^{48}$ and represent relatively simple molecules in terms of quick validation of parameters. When developing new parameters for the Cornell force field it is better to use analogy as much as possible. For that reason, predefined parameters and atom types were used when possible.<smiles>CN(C)O</smiles>

$\mathrm{MNO}$<smiles>CC(C)(C)N([O])C(C)(C)C</smiles>

TBNO

Figure 5.1. Chemical structures of Dimethoxyl Nitroxide Radical (MNO), and Di(tert-butyl)aminoxyl Radical (TBNO).

There are two characteristics of the NO• moiety that need special attention for the correct development of the force field. The first aspect is that the unpaired spin of the nonconjugated nitroxide is well localized on the diatomic $\mathrm{NO} \bullet$ moiety and leads to an $\mathrm{N}-\mathrm{O}$ length between single and double bond. The second aspect is that both the preference for a planar or pyramidal environment of the $\mathrm{N}$ atom and the partitioning of the spin density between the $\mathrm{N}$ 
and $\mathrm{O}$ atoms are strongly dependent on the nature of the substituents. Since the Cornell force field lacks suitable parameters for nitroxides, addition of two new atom types to the current force field was sufficient to account for the particular nature of the bond. The new atom types, referred to as NR and OR, and correspond to the nitrogen and oxygen atoms of the nitroxide group. The new force field parameters were developed by first, building the molecular geometry and second calculating associated point charges.

The missing parameters of the force field for nitroxides were obtained from ab initio SCF B3LYP theory level with 6-31G* basis set calculations. Equilibrium bond lengths and angles were taken from geometry-optimized structures. The bond force constants were determined from the Hessian matrix in internal coordinates and dihedral force constants were determined by fitting to the ab initio potential energy surface according to an earlier described scheme. ${ }^{25}$

The Cornell et al. (1995) force field originally was developed for nucleic acids and proteins. In addition to lacking nitroxide parameters, this force field required parameters for ethyne type carbons. This necessitated the development of another new atom type for a $s p$ hybridized carbon and its introduction to the Cornell force field. This parameter was adopted from the Tripos force field of Sybyl program (Tripos Inc., St. Louis, MO). It was modified By fitting the MM3 force field equations for $E_{\text {str }}$ and $E_{\text {bend }}$ energy terms to the appropriate terms in the force field equations represented in Amber. Thus, we were able to obtain force constants $k_{\theta}$ and $k_{r}$ together with equilibrium bond lengths and angles applicable in the framework of the Cornell force field. Newly developed force fields are summarized in the Appendix A. 


\subsubsection{Simulation Methods}

Starting structures of canonical B-form ss $\mathrm{T}_{15}$ and ds $\mathrm{A}_{15}: \mathrm{T}_{15}$ DNAs were built using biopolymer module of Sybyl 6.5 program. The docking module of Sybyl 6.5 package was used to merge $T_{15}$ to $A_{15}: T_{15}$ to form a triplex DNA structure of the sequence $T_{15}-A_{15}: T_{15}$. AMBER 5.0 package of programs was used for further work. Hydrogen atoms were added with the EDIT module of AMBER. These structures were minimized in vacuo to relax initial hydrogen atom positions after which sodium counterions $(\mathrm{Na}+)$ were added to neutralize the net charge. Then the nucleic acid was placed in a periodic box of explicit TIP3P water molecules extending to a distance of approximately $8 \AA$ in each direction from the DNA solute.

All other MM and MD calculations were carried out using the SANDER module of AMBER 5.0 with the Cornell et al. (1994) force field. Before initiating long MD runs, the system should be equilibrated. This implies first, relaxation of water molecules and counterions around the solute while the solute is constrained, adjustment of the box-size to achieve a constant water density, and second, gradual release of constraints on the solute until the whole system is free to move. Initial minimization was performed on water and counterions to a gradient convergence of $10^{-3} \mathrm{kcal} / \mathrm{mol} \cdot \AA$, with $500 \mathrm{kcal} / \mathrm{mol} \cdot \AA$ p positional restraints on nucleic acids. Then the water-counterion system was allowed to equilibrate using molecular dynamics for 25 picoseconds (ps).

This and subsequent MD simulations were performed in the isothermal isobaric ensemble $(300 \mathrm{~K}, 1 \mathrm{~atm})$ using periodic boundary conditions. The SHAKE algorithm was

applied to hydrogen atoms with a tolerance limit of $0.0005 \AA$, and a 2 femtosecond (fs) timestep. A $9 \AA$ cutoff was applied to Lennard-Jones interactions. Simulations were 
performed using Berendsen temperature coupling algorithm (with a time constant of $0.2 \mathrm{ps}$ ) and constant pressure with isotropic molecule based scaling (with a time constant of $0.2 \mathrm{ps}$ ). In this way two DNA strands were treated as if they were one, two or three different molecules and the shifting of these strands was avoided during equilibration. The non-bonded pair list was updated every 20 fs. Particle mesh-Ewald method (PME) for inclusion of longrange electrostatic interactions without truncation was followed. This method is a fast method for performing Ewald summation of Coulombic interactions. It means that the system is treated as if it was truly periodic and includes all electrostatic interactions within the unit cell (up to the cut-off) and with all image cells. A grid was constructed of cubes of approximate dimensions of $1.0 \AA^{3}$.

Next, the entire structure was minimized for 1000 steps with $25 \mathrm{kcal} / \mathrm{mole} \cdot \AA \AA$ of positional restrains on DNA followed by 3 ps dynamics run, which allowed water and $\mathrm{Na}+$ ions to relax around the solute. Subsequently, 600 steps of equilibration with gradual removal of positional restraints by $5 \mathrm{kcal} / \mathrm{mole} \cdot \AA$ A on DNA molecule for each run were performed. During the final dynamics the system was heated from $100 \mathrm{~K}$ to $300 \mathrm{~K}$ over 20 ps. Production runs of $1 \mathrm{~ns}$ duration, at constant temperature ( $\mathrm{T}=300 \mathrm{~K}$ ), were performed after the final dynamics step. After each run the PME box information was updated to match the final box coordinates from previous runs. The resulting trajectories were analyzed using Curves 5.0 as well as CARNAL, MDANAL, and rdparm modules of AMBER 5.0

The same protocol was used for simulations of modified $\mathrm{ss}_{7}{ }^{*} \mathrm{TT}_{7}$, ds $\mathrm{A}_{15}:\left(\mathrm{T}_{7}{ }^{*} \mathrm{TT}_{7}\right)$, and tx $\mathrm{d}\left(\mathrm{T}_{7}{ }^{5 \mathrm{sp}} \mathrm{TT}_{7}\right)-\mathrm{A}_{15}: \mathrm{T}_{15}$ DNAs, where ${ }^{*} \mathrm{~T}$ is either ${ }^{5 \mathrm{sp}} \mathrm{T}$ or ${ }^{6 \mathrm{sp}} \mathrm{T}$. One additional step was performed prior to equilibration for all modified DNAs. This was 5000 step minimization of 
structures in vacuo to minimize any possible steric effects from spin labels. For convenience the following notation will be used: $\mathrm{T}, \mathrm{AT}, \mathrm{TAT}, * \mathrm{~T}, \mathrm{~A} * \mathrm{~T}, * \mathrm{TAT},(*=5 \mathrm{sp}$ or $6 \mathrm{sp})$

\subsection{Results and Discussion}

Parameterization: In tables 5.1a and 5.1b are shown parameters of two nitroxide radical molecules obtained from optimization with quantum mechanics $(\mathrm{QM})$ and molecular mechanics (MM) methods implemented in gaussian98 and sander module of Amber programs, respectively. Results available from experimental data and QM calculation conducted by Barone et al. are also included in these Tables. Comparing the N-O bond lengths for both molecules obtained from QM and MM calculation, we can see that they fall well into the experimental range (1.26-1.29 $\AA$ ) reported by Baron et al.

Comparison of some of the parameters for DMNO and TBNO molecules shows very little discrepancy with the ones for experimental, B3LYP (by Barone et al.), and B3LYP (g98 calculation). It can be seen from the value of out-of-plane angle, $\tau=25.5^{\circ}$, that the geometry of DMNO after QM optimization has more pyramidal character than geometry of the molecule after MM minimization. The N2-O4 bond length shown in Table 5.1a in both cases falls in an experimental range. For the TBNO molecule geometrical parameters shown in Table 5.1b one can see that again QM gave more pyramidal geometry for the $\mathrm{N}-\mathrm{O}$ moiety than MM minimization. The out-of-plane values $\tau=0^{\circ}$ (from MM) and $\tau=20.5^{\circ}$ (from QM) obviously confirm that. But the comparison of $\tau$ for DMNO and TBNO indicates that former tends to be more planar than DMNO. This geometry can be driven by the fact that nitroxides are in most cases quasi-planar molecules, so that the orbital containing the odd number of electrons has an almost pure $\pi$ character. The structure of the radical either planar or bent, 
a)

\begin{tabular}{|c|c|c|c|c|}
\hline & $\operatorname{Exp}^{\mathrm{a}}$ & B3LYPa & $\mathbf{M M}^{\mathrm{b}}$ & B3LYP' \\
\hline \multicolumn{5}{|l|}{ Bonds $(\AA)$} \\
\hline $\mathrm{N} 2-\mathrm{O} 4$ & 1.28 & 1.282 & 1.278 & 1.286 \\
\hline $\mathrm{N} 2-\mathrm{C} 1$ & - & 1.463 & 1.469 & 1.548 \\
\hline \multicolumn{5}{|l|}{ Angles $\left({ }^{\circ}\right)$} \\
\hline C1-N2-O4 & - & - & 119.31 & 117.55 \\
\hline C3-N2-O4 & - & 119.4 & 119.37 & 117.64 \\
\hline C1-N2-C3 & 118.9 & 115.5 & 121.31 & 118.97 \\
\hline \multicolumn{5}{|l|}{ Torsion $\left({ }^{\circ}\right)$} \\
\hline O4-N2-C1-H5 & $\overline{-1}$ & $\overline{-1}$ & 0.02 & 38.48 \\
\hline O4-N2-C1-H6 & - & - & 0.00 & 11.10 \\
\hline \multicolumn{5}{|l|}{ Out-of-plane angle $\left(^{\circ}\right)$} \\
\hline$\tau$ & - & - & 0.00 & 25.5 \\
\hline
\end{tabular}

b)

\begin{tabular}{|c|c|c|c|c|}
\hline & Exp & B3LYP & MM & B3LYP \\
\hline \multicolumn{5}{|l|}{ Bonds $(\AA)$} \\
\hline $\mathrm{N} 2-\mathrm{O} 4$ & $1.280 \pm 0.02$ & 1.287 & 1.281 & 1.288 \\
\hline $\mathrm{N} 2-\mathrm{C} 1$ & $1.512 \pm 0.02$ & 1.511 & 1.589 & 1.517 \\
\hline $\mathrm{N} 2-\mathrm{C} 3$ & - & - & 1.588 & 1.517 \\
\hline \multicolumn{5}{|l|}{ Angles $\left({ }^{\circ}\right)$} \\
\hline C1-N2-O4 & - & - & 118.25 & 112.75 \\
\hline C3-N2-O4 & - & - & 118.26 & 115.86 \\
\hline C1-N2-C3 & $136 \pm 3$ & 127.9 & 123.49 & 128.12 \\
\hline \multicolumn{5}{|l|}{ Torsion $\left({ }^{\circ}\right)$} \\
\hline O4-N2-C1-C5 & $137 \pm 2$ & 138.3 & 136.00 & 173.70 \\
\hline \multicolumn{5}{|l|}{ Out-of-plane angle $\left(^{\circ}\right)$} \\
\hline$\tau$ & - & - & 0.00 & 20.5 \\
\hline
\end{tabular}

Table 5.1. Geometrical parameters for a) Dimethyl Nitroxide Radical (DMNO) and b) Di(tertbutyl)aminoxyl Radical (TBNO).

\footnotetext{
${ }^{\mathrm{a}}$ From reference \# 7

${ }^{\mathrm{b}}$ From optimization from Sander using modified Cornell 94 force fields
} 
depending on whether stabilization from partial $\pi$ bond is sufficient to retain a pure $\mathrm{sp}^{2}$ hybridization of the nitrogen. The competition between $\pi$ bonding and the preference of nitrogen for $\mathrm{sp}^{3}$ hybridization explains the occurrence of both planar and pyramidal structures depending on molecular topologies.

Because of the lack of experimental data for $5 \mathrm{sp}$ and $6 \mathrm{sp}$ nitroxide molecules only MM and QM geometry-optimized parameters involving NO moiety are compared (Table 5.2). Geometry optimization was carried out with sander using modified Cornell force fields (MM)

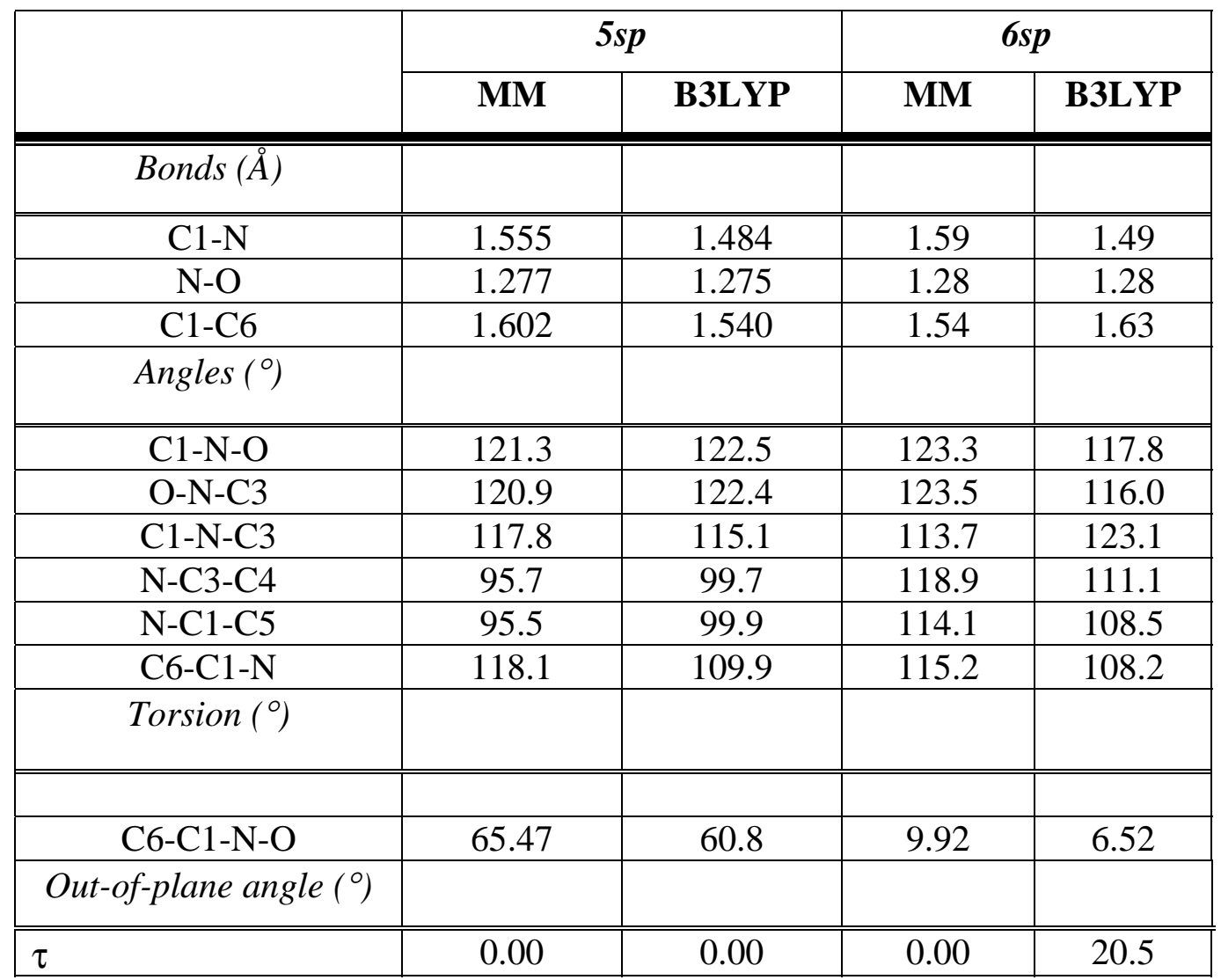

Table 5.2. Geometrical parameters for $5 \mathrm{sp}$ and $6 \mathrm{sp}$ nitroxide molecues.

\footnotetext{
${ }^{\mathrm{c}}$ From QM calculation by g98 program
} 
and with gaussian98 (QM). The bond length for NO moiety for both 5sp and 6sp nitroxides are well reproduced both $\mathrm{MM}$ and QM calculation, and they are in good agreement with experimental values obtained for the model compounds (MNO and TBNO).

The C-N-C angle for DMNO and TBNO molecules is larger than the angle O-N-C. The out-of-plane angle of the NO moiety depends on the value of C-N-C angle. It is also larger for QM optimized 6sp molecule. As for the out-of-plane angles, in general, increase in $\tau$ implies decrease in the $\mathrm{C}-\mathrm{N}-\mathrm{C}$ angle. This does not offer a rationalization for the planarity of 5-membered ring nitroxide molecule, where the $\mathrm{C}-\mathrm{N}-\mathrm{C}$ angle is $115^{\circ}$. For this molecule $\mathrm{C}$ $\mathrm{N}-\mathrm{C}$ angle $\left(122^{\circ}\right)$ is smaller than $\mathrm{O}-\mathrm{N}-\mathrm{C}$ one $\left(115^{\circ}\right)$, which is due to the fact that the angles in 5-membered ring are constrained and can not deviate too much from the value of $108^{\circ}$, characterizing a regular pentagon. The geometry of the 6-membered ring nitroxide tends to maintain more planar character when minimized with sander as opposed to the quantum mechanical optimization. This can be explained by the parameters being more favorable for handling flat rings.

Partial charges for both $5 \mathrm{sp}$ and $6 \mathrm{sp}$ nitroxides are shown in the Table 5.3. They are derived by fitting 6-31G* electrostatic potentials with RESP algorithm implemented in the Amber program. These charges are added only to the modified part of the thymidine, keeping the thymidine charges as in the Amber library. To make sure that the modification did not have a significant effect on the nucleotide charges we have also calculated electrostatic potentials for the $5 \mathrm{sp}$ and $6 \mathrm{sp}$ modified thymidine (DTM). Charges fitted with the RESP algorithm of the nucleotide fragment are compared to the charges of the thymidine from the amber database (Table 5.4). 

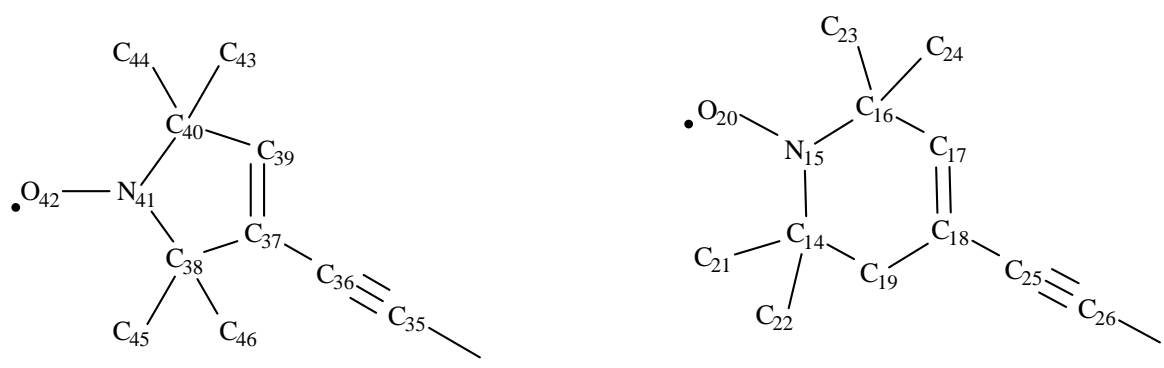

\begin{tabular}{|l|l|l|l|}
\hline 5sp & \multicolumn{3}{l|}{ 6sp } \\
\hline \hline Atom & Charge & Atom & Charge \\
\hline C38 & 0.1705 & C14 & 0.13647 \\
\hline N41 & 0.129928 & N15 & 0.164088 \\
\hline C40 & 0.1148 & C16 & 0.138739 \\
\hline C39 & -0.1172 & C17 & -0.197288 \\
\hline H59 & 0.1393 & H71 & 0.138191 \\
\hline C37 & 0.013 & C18 & 0.049737 \\
\hline & & C19 & -0.024656 \\
\hline & & H91 & 0.044053 \\
\hline & & H92 & 0.044053 \\
\hline O42 & -0.339 & O20 & -0.304179 \\
\hline C43 & -0.1539 & C21 & -0.132922 \\
\hline H47 & 0.0513 & H11 & 0.049738 \\
\hline H48 & 0.0593 & H12 & 0.049738 \\
\hline H49 & 0.0708 & H13 & 0.049738 \\
\hline C44 & -0.161 & C22 & -0.256358 \\
\hline H50 & 0.0606 & H21 & 0.081414 \\
\hline H51 & 0.0724 & H22 & 0.081414 \\
\hline H52 & 0.0529 & H23 & 0.081414 \\
\hline C45 & -0.1567 & C23 & -0.213413 \\
\hline H53 & 0.0623 & H31 & 0.072138 \\
\hline H54 & 0.0613 & H32 & 0.072138 \\
\hline H55 & 0.0642 & H33 & 0.072138 \\
\hline C46 & -0.1626 & C24 & -0.136367 \\
\hline H56 & 0.0656 & H41 & 0.054174 \\
\hline H57 & 0.064 & H42 & 0.054174 \\
\hline H58 & 0.063 & H43 & 0.054174 \\
\hline C35 & -0.11034 & C25 & -0.10922 \\
\hline C36 & -0.11034 & C26 & -0.10922 \\
\hline & & & \\
\hline
\end{tabular}

Table 5.3. Partial electronic charges of $5 \mathrm{sp}$ and $6 \mathrm{sp}$ fragments of modified thymidine evaluated by fitting the 6-31G* electrostatic potentials with the RESP algorithm. 


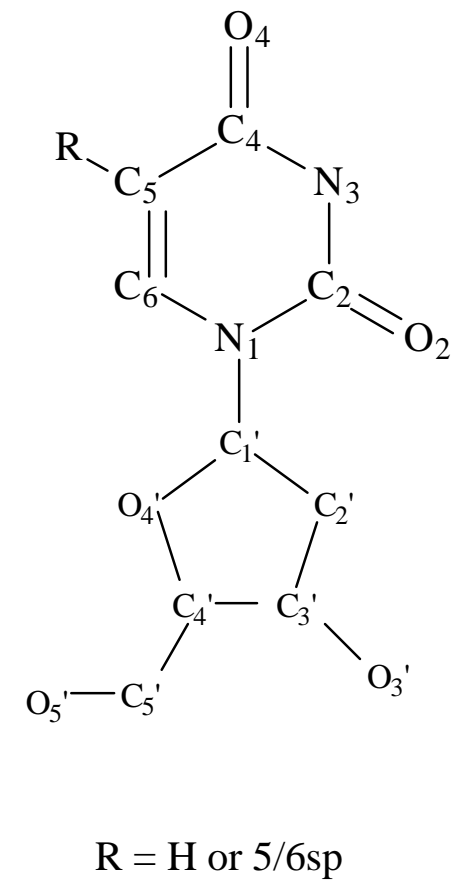

\begin{tabular}{|c|c|c|}
\hline & DT 6-31G & DTM 6-31G* \\
\hline Atom & \multicolumn{2}{|c|}{ Charge } \\
\hline O5' & -0.6318 & -0.54717 \\
\hline C5' & -0.0069 & 0.032356 \\
\hline $1 \mathrm{H}^{\prime}$ & 0.0754 & 0.086805 \\
\hline $2 \mathrm{H} 5{ }^{\prime}$ & 0.0754 & 0.086805 \\
\hline C4' & 0.1629 & 0.079405 \\
\hline H4' & 0.1176 & 0.09679 \\
\hline O4' & -0.3691 & -0.344405 \\
\hline C1' & 0.068 & 0.169684 \\
\hline $\mathrm{H} 1^{\prime}$ & 0.1804 & 0.160121 \\
\hline N1 & -0.0239 & -0.067217 \\
\hline C6 & -0.2209 & -0.067098 \\
\hline $\mathrm{H} 6$ & 0.2607 & 0.163613 \\
\hline C5 & 0.0025 & -0.002066 \\
\hline C4 & 0.5194 & 0.436842 \\
\hline $\mathrm{O} 4$ & -0.5563 & -0.463099 \\
\hline N3 & -0.434 & -0.357077 \\
\hline $\mathrm{H} 3$ & 0.342 & 0.311138 \\
\hline C2 & 0.5677 & 0.451239 \\
\hline $\mathrm{O} 2$ & -0.5881 & -0.495108 \\
\hline C3' & 0.0713 & 0.215217 \\
\hline H3' & 0.0985 & 0.023812 \\
\hline C2' & -0.0854 & -0.143885 \\
\hline $1 \mathrm{H}^{\prime}$ & 0.0718 & 0.047027 \\
\hline $2 \mathrm{H}^{\prime}$ & 0.0718 & 0.047027 \\
\hline O3' & -0.6549 & -0.594283 \\
\hline
\end{tabular}

Table 5.4. Partial electronic charges of thymidine fragment DT from Amber compared to the thymidine fragment of modified thymidine DTMevaluated by fitting the $6-31 \mathrm{G}^{*}$ electrostatic potentials with the RESP algorithm. 
Strand analysis: The root-mean-square deviations (RMSD) of snapshots from initial structures of double and triple stranded DNAs are shown in Figure 5.2a and 5.2b. By comparing unmodified and both 5sp and 6sp modified duplexes (Figure 5.2a) we notice that AT fluctuates more throughout the course of $1 \mathrm{~ns}$ trajectory than $\mathrm{A}^{5 \mathrm{sp}} \mathrm{T}$ and $\mathrm{A}^{6 \mathrm{sp}} \mathrm{T}$. The range of fluctuations is larger for $\mathrm{AT}$ rather than for $\mathrm{A}^{5 \mathrm{sp}} \mathrm{T}$ and $\mathrm{A}^{6 \mathrm{sp}} \mathrm{T}$ DNAs. It is reflected in the

a)

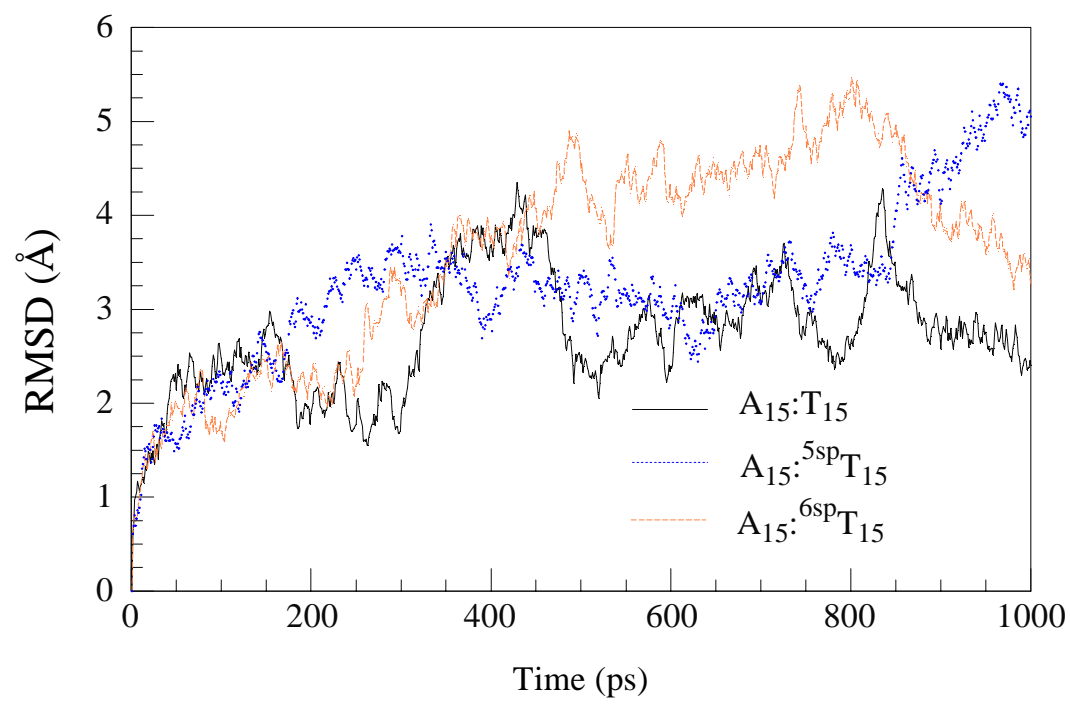

b)

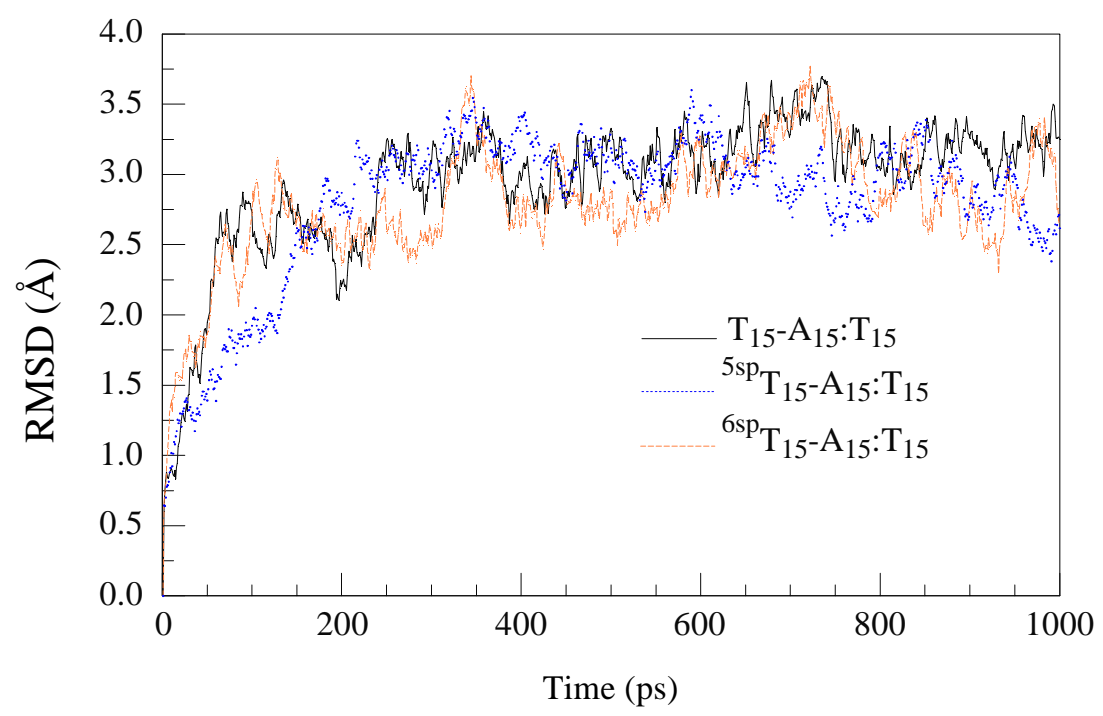

Figure 5.2. Root-mean-square deviation of a) $A_{15}: T_{15}$ (solid), $A_{15}:{ }^{5 \mathrm{pp}} \mathrm{T}_{15}$ (dotted) and $\mathrm{A}_{15}:{ }^{6 \mathrm{sp}} \mathrm{T}_{15}$ (dashed) duplex DNA and b) $\mathrm{T}_{15}-\mathrm{A}_{15}: \mathrm{T}_{15}$ (solid), ${ }^{5 \mathrm{sp}} \mathrm{T}_{15}-\mathrm{A}_{15}: \mathrm{T}_{15}$ (dotted) and ${ }^{6 \mathrm{sp}} \mathrm{T}_{15}-\mathrm{A}_{15}: \mathrm{T}_{15}$ (dashed) triplex DNA atoms from the initial structures as a function of time. 
in the jump around 200-500 ps interval and also at $800-900$ ps. In contrast, the range of fluctuations is rather small for $\mathrm{A}^{5 \mathrm{sp}} \mathrm{T}$ and $\mathrm{A}^{6 \mathrm{sp}} \mathrm{T}$ DNAs and they do not show big jumps until about $800-850$ ps. This rather sharp change might be the indication of possible conformational change occurring in molecules. These changes may be driven by presence of the spin-label in both DNAs as it tries to accommodate itself in the major groove of the DNA. Evidently, longer production runs will be required to determine if such processes are happening in these molecules.

The RMSD between average structures of both AT and $\mathrm{A}^{5 \mathrm{sp}} \mathrm{T}$ as well as AT and $\mathrm{A}^{6 \mathrm{sp}} \mathrm{T}$ duplexes are compared and found to be $1.25 \AA$ and $1.21 \AA$ respectively. It is not always desirable to use the average structure for the analyses of DNA, since it represents the static picture of the molecule. Considering DNA as a dynamic molecule it is better to use a structure that would best represent all other ones in the trajectory file. Thus, we also conducted cluster analysis, which seeks for the "best" representative structure from a dynamics trajectory within the specified cutoff of $2.5 \AA$. We found that RMSD between the "best" structures is $3.45 \AA$ for $\mathrm{AT}$ and $\mathrm{A}^{5 \mathrm{sp}} \mathrm{T}$ and it is $4.49 \AA$ for $\mathrm{AT}$ and $\mathrm{A}^{6 \mathrm{sp}} \mathrm{T}$. This quite a large difference between two structures suggest that there are changes in minor and major grooves, backbone and possibly in some other structural parameters, which will be discussed later in the text.

In contrast to the duplex, there are no large changes in RMSD fluctuations of both 5sp and 6sp modified and unmodified triplexes. Initial fluctuations are large in magnitude for all three structures for up to 200 ps. After 200 ps, all three structures show quite small fluctuations, within $1 \AA$ range, around steady values. This, in turn, suggests that the presence of modified base does not significantly alter the overall structure of the triplex. Also, as 
opposed to the duplex $1 \mathrm{~ns}$ production run is sufficient and that there is unlikely that we could see any conformational changes happening in these structures after $1 \mathrm{~ns}$. RMSD values between the average $(0.76 \AA$ and $0.92 \AA)$ and the "best" structures $(1.63 \AA$ and $1.92 \AA)$ between unmodified and $5 \mathrm{sp}$ modified triplexes and unmodified and 6sp modified, respectively, also support that statement.

It is also instructive to look at the RMSD with respect to the average structure during the course of the MD run. Figure 5.3 represents the RMSD plot of duplexes (a) and triplexes (b) with respect to the their mean structures. It is evident that duplexes have larger RMSD during the course of the simulation from the average structure than the triplexes. 
a)

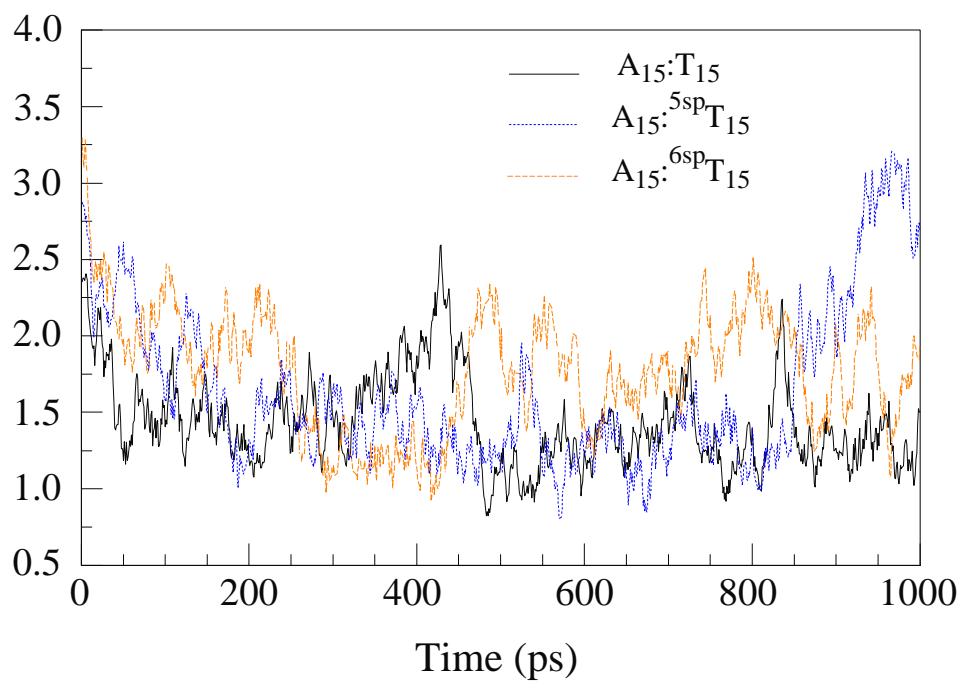

b)

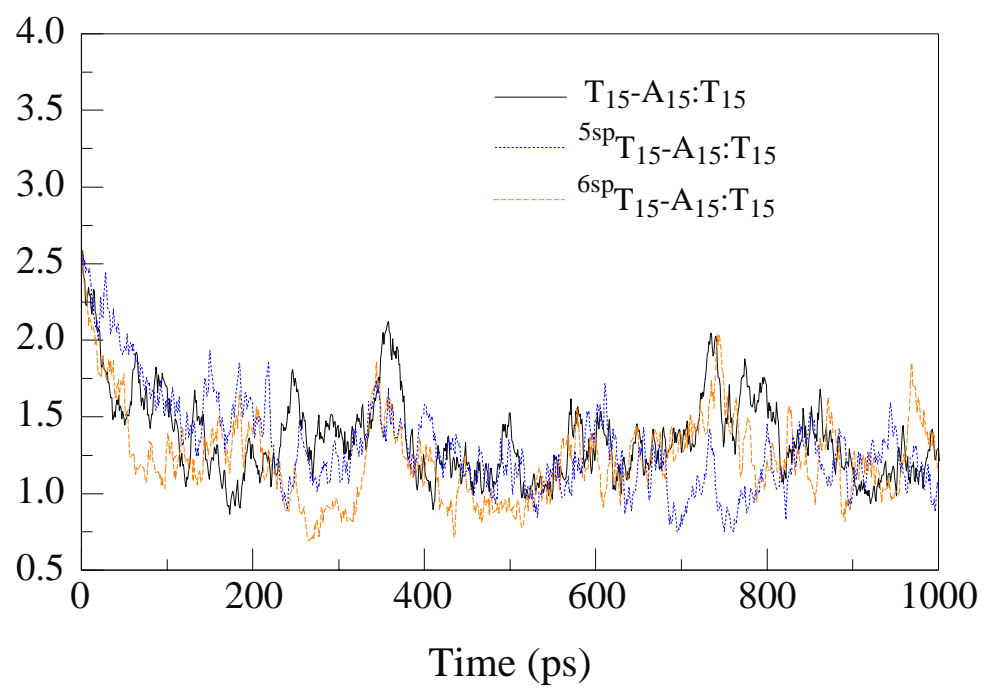

Figure 5.3. Root-mean-square deviation of a) $\mathrm{A}_{15}: \mathrm{T}_{15}$ (solid), $\mathrm{A}_{15}:{ }^{5 \mathrm{sp}} \mathrm{T}_{15}$ (dotted) and $\mathrm{A}_{15}:{ }^{6 \mathrm{sp}} \mathrm{T}_{15}$ (dashed) duplex DNA and b) $\mathrm{T}_{15}-\mathrm{A}_{15}: \mathrm{T}_{15}$ (solid), ${ }^{5 \mathrm{sp}} \mathrm{T}_{15}-\mathrm{A}_{15}: \mathrm{T}_{15}$ (dotted) and ${ }^{6 \mathrm{sp}} \mathrm{T}_{15}-\mathrm{A}_{15}: \mathrm{T}_{15}$ (dashed) triplex DNA atoms to their respective mean structures as a function of time. 
To investigate further, we looked at the evolution of structural parameters determining the backbone conformations and global parameters of all six DNAs during the course of the simulation. These parameters are compared with canonical B- and A-DNA conformations and are summarized in Tables 5.5 - 5.9 and Figures 5.4 - 5.7 for all duplexes and triplexes. The average values of torsional angles of canonical DNA helices are given in the Table 5.5 below and Figure 5.4a illustrates them.

\begin{tabular}{|c|c|c|c|c|c|c|}
\hline Type & $\boldsymbol{\alpha}$ & $\boldsymbol{\beta}$ & $\boldsymbol{\gamma}$ & $\boldsymbol{\delta}$ & $\boldsymbol{\varepsilon}$ & $\boldsymbol{\xi}$ \\
\hline \hline $\mathrm{A}$ & -50 & 172 & 41 & 79 & -146 & -78 \\
\hline $\mathrm{B}$ & -41 & 136 & 38 & 139 & -133 & -157 \\
\hline
\end{tabular}

Table 5.5. Average torsion angles for canonical DNA helices (in ${ }^{\circ}$ )

At this point it will be appropriate to describe the way some of the groove parameters have been defined and measured ${ }^{49}$ (Figure 5.4b). First, the axis is defined, which is measured as the cubic spline passing through the set of axis reference points $p_{i}$, and tangential to the local helical axis $\mathbf{U}_{\mathrm{i}}$. There is also a definition of the pseudodyad vector $\mathbf{V}_{\mathrm{i}}$ at each point pi, which points into the center of the major groove. $\mathbf{V}_{\mathrm{i}}$ is always perpendicular to the local helical axis $\mathbf{U}_{\mathrm{i}}$. To calculate the groove width at some point $\mathrm{O}$ the plane is rotated around vector $\mathbf{V}$ and intersection points $I_{1}$ and $I_{2}$ of the two backbone curves with this plane are calculated. The difference of these points $L=\left|I_{1}-I_{2}\right|$ is a minimum and maximum in the minor and major grooves, respectively. To define the depths of the grooves, one considers single 
points $\mathrm{F}_{\mathrm{M}}$ (major) and $\mathrm{F}_{\mathrm{m}}$ (minor) on the $\mathbf{V}$ axis, which corresponds to the mean position at which the van der Waals surfaces of the base pairs cut this axis.

a)

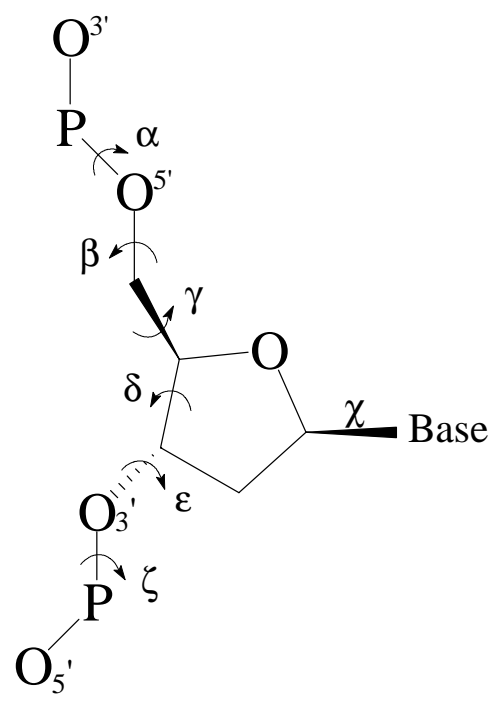

b)

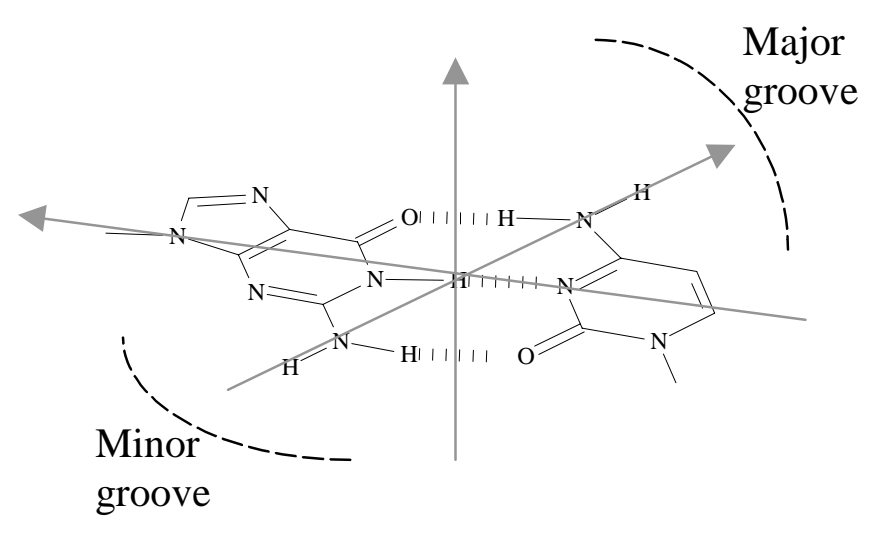

Figure 5.4. Definition of a) torsional angles and b) global groove parameters.

Looking at the groove parameters of all three duplexes summarized in Table 5.6 we can see consistent increase in the major groove width of both modified DNAs compared to unmodified (Table 5.6a). This can be explained by the presence of the spin label in the major groove. As the $5 \mathrm{sp}$ label is smaller than the $6 \mathrm{sp}$, one would expect to see more widening of the major groove of the ${ }^{6 \mathrm{sp}} \mathrm{TA}$, which is actually a case. The depth of the major groove is also changing with the presence of the spin label (Table 5.6b). 


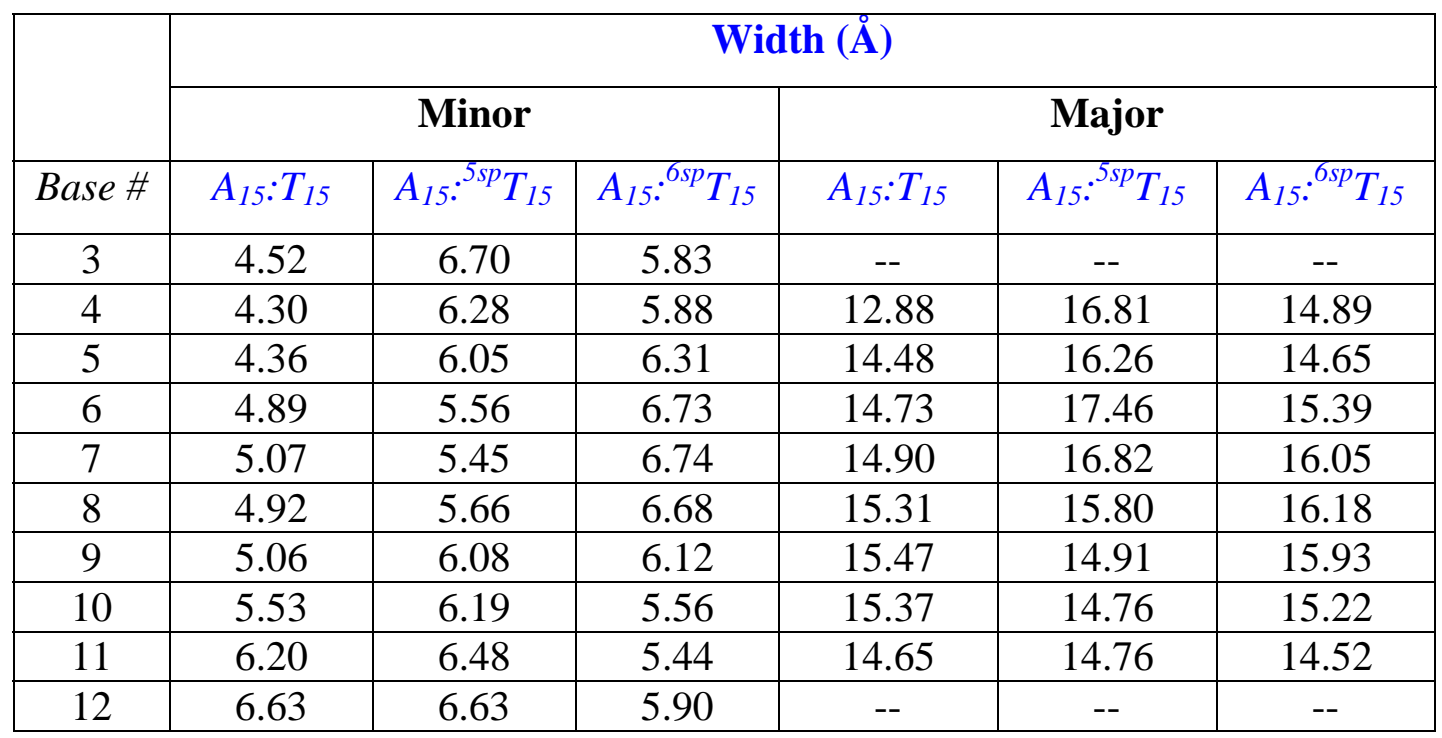

Table 5.6a. Global groove width parameters for unmodified and $5 \mathrm{sp}$ and $6 \mathrm{sp}$ modified DUPLEXES.

\begin{tabular}{|c|c|c|c|c|c|c|}
\hline \multirow[b]{3}{*}{ Base \# } & \multicolumn{6}{|c|}{ Depth $(\AA)$} \\
\hline & \multicolumn{3}{|c|}{ Minor } & \multicolumn{3}{|c|}{ Major } \\
\hline & $A_{15}: T_{15}$ & $A_{15}{ }^{.5 p} T_{15}$ & $A_{15}{ }^{. s p} T_{15}$ & $A_{15}: T_{15}$ & $A_{15}{ }^{.5 s p} T_{15}$ & $A_{15 .}{ }^{. s p} T_{15}$ \\
\hline 3 & 4.82 & 3.95 & 3.44 & -- & - & -- \\
\hline 4 & 4.79 & 3.99 & 3.57 & 7.00 & 3.57 & 6.90 \\
\hline 5 & 4.74 & 4.39 & 3.6 & 7.07 & 7.37 & 7.98 \\
\hline 6 & 4.55 & 4.58 & 3.76 & 7.3 & 5.15 & 7.82 \\
\hline 7 & 4.66 & 4.55 & 3.82 & 7.25 & 6.70 & 7.44 \\
\hline 8 & 4.61 & 4.53 & 3.85 & 7.12 & 6.66 & 7.36 \\
\hline 9 & 4.46 & 4.47 & 4.05 & 7.44 & 7.61 & 6.2 \\
\hline 10 & 4.38 & 4.31 & 4.2 & 6.88 & 7.94 & 6.86 \\
\hline 11 & 4.07 & 4.12 & 4.19 & 7.67 & 7.94 & 7.28 \\
\hline 12 & 3.92 & 4.02 & 4.14 & -- & -- & -- \\
\hline
\end{tabular}

Table 5.6b. Global groove depth parameters for unmodified and 5sp and 6sp modified DUPLEXES. 
Similar pattern of increasing of the major groove width can be monitored for spinlabel modified triplexes (Figure 5.7a). This change is again promoted by the presence of the spin label in the groove. There is also increase in the major groove depth of modified triplexes (Figure 5.7b). At the same time the minor groove depth decreases in response to the increasing both major width and depth. All these changes in the groove parameters lead to the changes in diameter also, which can be seen from Table 5.8 and accompanying Figure 5.5.

The diameter of both spin-labeled duplexes and triplexes is increased. Changes in diameter of the modified duplexes indicate the tendency of these structures to undergo conformational change. These changes could also be seen from the RMSD fluctuations with respect to starting structure (Figure 5.2) and they lead to the appearance of the internal diameter of the duplex. The presence of the internal diameter implies that the duplex is moving towards the A-DNA conformation. Changes in diameter can be monitored over the course of the simulation (data not shown). 


\begin{tabular}{|c|c|c|c|c|c|c|}
\hline \multirow[b]{3}{*}{ Base \# } & \multicolumn{6}{|c|}{ Width $(\AA)$} \\
\hline & \multicolumn{3}{|c|}{ Minor } & \multicolumn{3}{|c|}{ Major } \\
\hline & $T-A: T$ & ${ }^{S s p} T-A: T$ & ${ }^{6 s p} T-A: T$ & $T-A: T$ & ${ }^{{ }^{s s p}} T-A: T$ & ${ }^{b s p} T-A: T$ \\
\hline 3 & -- & -- & -- & -- & 9.5 & 9.81 \\
\hline 4 & 16.35 & -- & -- & 9.05 & 9.19 & 9.65 \\
\hline 5 & 14.55 & 15.28 & 15.10 & 8.98 & 8.65 & 9.49 \\
\hline 6 & 13.55 & 15.18 & 15.29 & 6.13 & 8.76 & 9.59 \\
\hline 7 & 14.77 & 15.10 & 15.43 & 6.24 & 9.93 & 10.06 \\
\hline 8 & 15.08 & 15.22 & 15.60 & 7.53 & 10.03 & 9.65 \\
\hline 9 & 14.90 & 15.28 & 15.30 & 8.05 & 9.73 & 9.02 \\
\hline 10 & 14.90 & 15.34 & 14.92 & 8.42 & 9.36 & 8.56 \\
\hline 11 & 15.32 & 15.33 & 14.75 & 9.55 & 9.14 & 8.2 \\
\hline 12 & -- & -- & 9.87 & 9.86 & -- & -- \\
\hline
\end{tabular}

Table 5.7a. Global groove width parameters for unmodified and $5 \mathrm{sp}$ and $6 \mathrm{sp}$ modified TRIPLEXES.

\begin{tabular}{|c|c|c|c|c|c|c|}
\hline \multirow[b]{3}{*}{ Base \# } & \multicolumn{6}{|c|}{ Depth $(\AA)$} \\
\hline & \multicolumn{3}{|c|}{ Minor } & \multicolumn{3}{|c|}{ Major } \\
\hline & $T-A: T$ & ${ }^{5 s p} T-A \cdot T$ & ${ }^{6 s p} T-A: T$ & $T-A: T$ & ${ }^{S s p} T-A: T$ & ${ }^{{ }^{s p}} T-A: T$ \\
\hline 3 & -- & -- & -- & -- & 5.31 & 4.25 \\
\hline 4 & 2.87 & -- & -- & 9.05 & 7.92 & 6.95 \\
\hline 5 & 4.28 & 2.13 & 3.02 & 8.98 & 7.81 & 6.99 \\
\hline 6 & 4.24 & 2.22 & 2.21 & 6.13 & 8.06 & 7.3 \\
\hline 7 & 4.28 & 2.53 & 2.39 & 6.24 & 7.41 & 7.25 \\
\hline 8 & 3.08 & 2.44 & 2.01 & 7.53 & 7.33 & 7.64 \\
\hline 9 & 2.08 & 2.60 & 1.73 & 8.05 & 7.52 & 8.07 \\
\hline 10 & 2.15 & 2.82 & 2.50 & 8.42 & 7.93 & 8.28 \\
\hline 11 & 0.58 & 3.49 & 3.53 & 9.55 & 8.48 & 8.79 \\
\hline 12 & -- & -- & 3.65 & 9.86 & -- & -- \\
\hline
\end{tabular}

Table 5.7b. Global groove depth parameters for unmodified and 5sp and 6sp modified TRIPLEXES. 


\section{Diameter}

\begin{tabular}{|c|c|c|c|}
\hline \hline & $A_{15}: T_{15}$ & $A_{15}{ }^{5 s p} T_{15}$ & $A_{15}{ }^{6 s p} T_{15}$ \\
\hline Base \# & & & \\
\hline 3 & 20.38 & 21.51 & 20.26 \\
\hline 4 & 20.41 & 21.82 & 20.65 \\
\hline 5 & 20.32 & 21.45 & 20.81 \\
\hline 6 & 20.55 & 21.46 & 21.03 \\
\hline 7 & 20.56 & 21.46 & 21.13 \\
\hline 8 & 20.38 & 21.61 & 21.17 \\
\hline 9 & 20.34 & 21.52 & 21.24 \\
\hline 10 & 20.30 & 21.31 & 21.08 \\
\hline 11 & 20.10 & 21.33 & 20.84 \\
\hline 12 & 20.03 & 21.31 & 20.82 \\
\hline
\end{tabular}

\begin{tabular}{|c|c|c|}
\hline$T-A: T$ & ${ }^{\text {Ssp }} T-A: T$ & ${ }^{\text {Gs }} T-A: T$ \\
\hline & & \\
\hline 19.84 & 20.77 & 20.36 \\
\hline 19.43 & 20.73 & 20.42 \\
\hline 18.60 & 20.64 & 20.36 \\
\hline 18.28 & 20.64 & 20.37 \\
\hline 19.38 & 20.48 & 20.44 \\
\hline 19.52 & 20.36 & 20.42 \\
\hline 19.36 & 20.25 & 20.32 \\
\hline 19.29 & 20.02 & 20.11 \\
\hline 19.38 & 19.83 & 19.94 \\
\hline 19.32 & 19.48 & 19.64 \\
\hline
\end{tabular}

Table 5.8. Diameter for unmodified and 5sp and 6sp modified DUPLEXES and TRIPLEXES.

a)

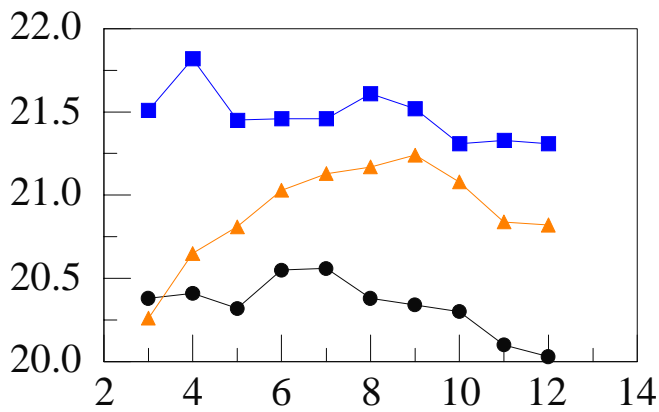

Base \# b)

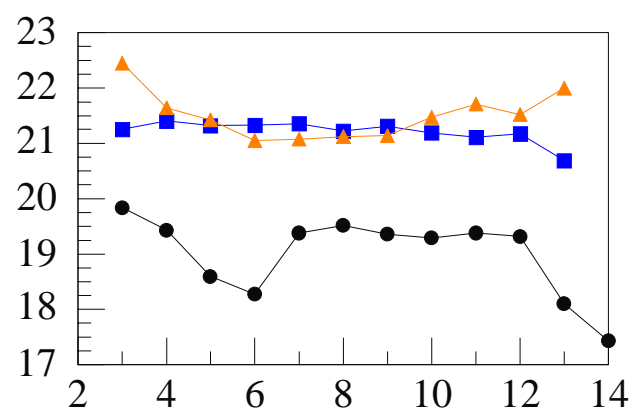

Base \#

Figure 5.5. Average diameter for unmodified $(\bullet), 5 \operatorname{sp}(\square)$ and $6 \operatorname{sp~}(\Delta)$ modified a) duplexes and b) triplexes. 
There is a concern that the presence of the spin probe alters the conformation of the DNAs under study. It may introduce some bending of the DNA due to the stacking of the acetylene tether with adjacent nucleotides or, on the contrary, lead to the elimination of the bending of the DNA. It is important to know how the conformation is altered so that studies using spin probe labeled DNAs can be related to the corresponding unlabeled DNAs.

The sequences responsible for DNA bending were identified as oligo (dA):oligo (dT) tracts. From MD studies we compared the bending of unmodified duplex with $5 \mathrm{sp}$ and $6 \mathrm{sp}$ modified ones. We can see that overall axis bend of the local helical axis UU is larger by about $8 \%$ for AT than for ${ }^{5 \mathrm{sp}} \mathrm{TA}$ and by $28 \%$ than that for ${ }^{6 \mathrm{sp}} \mathrm{TA}$. This actually is an indication of straightening of both modified DNAs as opposed to unlabeled AT. These changes in the axis bend along with changes of the diameter do suggest a tendency of the modified duplexes to go towards the A-DNA conformation. Longer dynamics runs will definitely clarify this finding.

Changes in the global axis parameters for the triplexes are not consistent with the results obtained for duplexes. One would expect to have less bending in the triplex because of the presence of the third strand. These discrepancies may rise from the way the Curves program interprets unusual DNA structures, particularly triplex DNAs. More in depth investigation of these parameters would need to be done in the future.

Figures 5.6 and 5.7 show the average backbone angles for duplexes and triplexes, respectively. The torsional angles $\delta$ and $\xi_{\text {of }}$ both modified duplexes tend to the values that are closer to A-DNA conformation (refer to Table 5.5). While the other angles of both spinlabel modified duplexes seem to have similar values as unmodified duplex. 


\begin{tabular}{|c|c|c|c|}
\hline & $A: T$ & $A:^{S s p} T$ & $A:{ }^{6 s p} T$ \\
\hline Curvature & & & \\
\hline Path length & 47.89 & 45.83 & 44.72 \\
\hline End-to-end & 47.25 & 45.69 & 44.62 \\
\hline Shortening & $1.33 \%$ & $0.29 \%$ & $0.23 \%$ \\
\hline \multirow{3}{*}{ Overall axis bend } & $\mathrm{UU}=13.62$ & $\mathrm{UU}=12.56$ & $\mathrm{UU}=10.62$ \\
& $\mathrm{PP}=14.71$ & $\mathrm{PP}=10.67$ & $\mathrm{PP}=11.07$ \\
\hline
\end{tabular}

\begin{tabular}{|c|c|c|c|}
\hline & $T-A: T$ & ${ }^{S s p} T-A: T$ & ${ }^{{ }^{s p}} T-A: T$ \\
\hline Curvature & & & \\
\hline Path length & 21.72 & 19.27 & 21.40 \\
\hline End-to-end & 18.22 & 15.84 & 16.85 \\
\hline Shortening & $16.11 \%$ & $17.79 \%$ & $21.26 \%$ \\
\hline \multirow{2}{*}{ Overall axis bend } & $\mathrm{UU}=28.91$ & $\mathrm{UU}=21.22$ & $\mathrm{UU}=32.71$ \\
& $\mathrm{PP}=35.39$ & $\mathrm{PP}=75.82$ & $\mathrm{PP}=78.57$ \\
\hline
\end{tabular}

Table 5.9. Global axis parameters for unmodified and $5 \mathrm{sp}$ and $6 \mathrm{sp}$ modified ds and tx DNAs.

For triplexes we do not see much differences in torsional angles (Figure 5.7). It would be difficult to see any changes during $1 \mathrm{~ns}$ molecular dynamics run because of the rigidity of the triplex. In the triplex not only the spin label needs to be accommodated in the groove, but also an additional strand and the changes of the backbone torsional angles would need to be quite large. This can not be achieve at such short dynamics runs. 

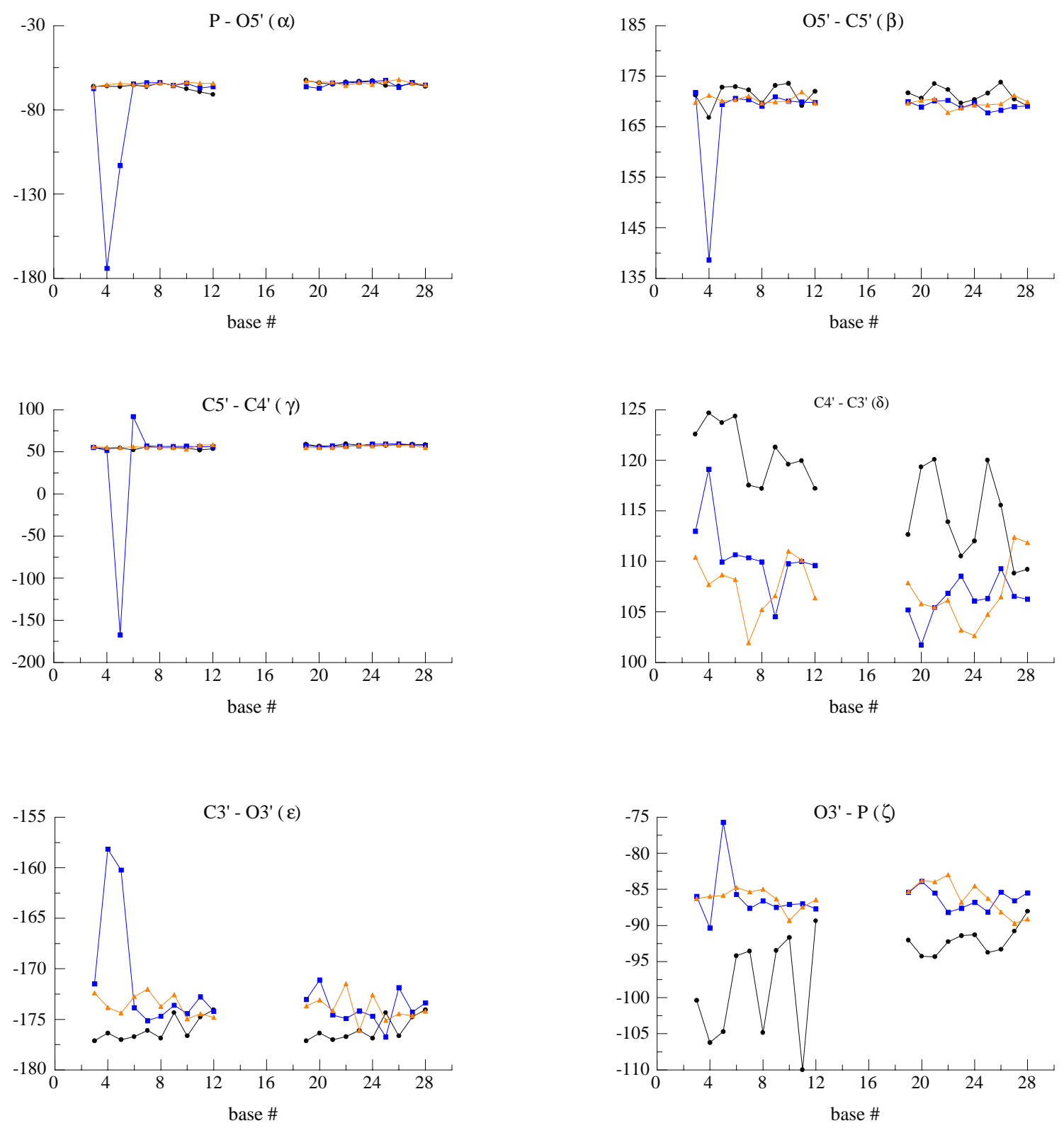

Figure 5.6. Average backbone parameters for double stranded DNAs: $A_{15}: T_{15}(\bullet), A_{15}:{ }^{5 s p} T_{15}(\square)$ and $\mathrm{A}_{15}:{ }^{6 \mathrm{sp}} \mathrm{T}_{15}(\triangle)$. Bases 3-12 are the A-strand, bases 19-28 are the $* \mathrm{~T}$ strand $(*=5 \mathrm{sp}$ or $6 \mathrm{sp})$. 

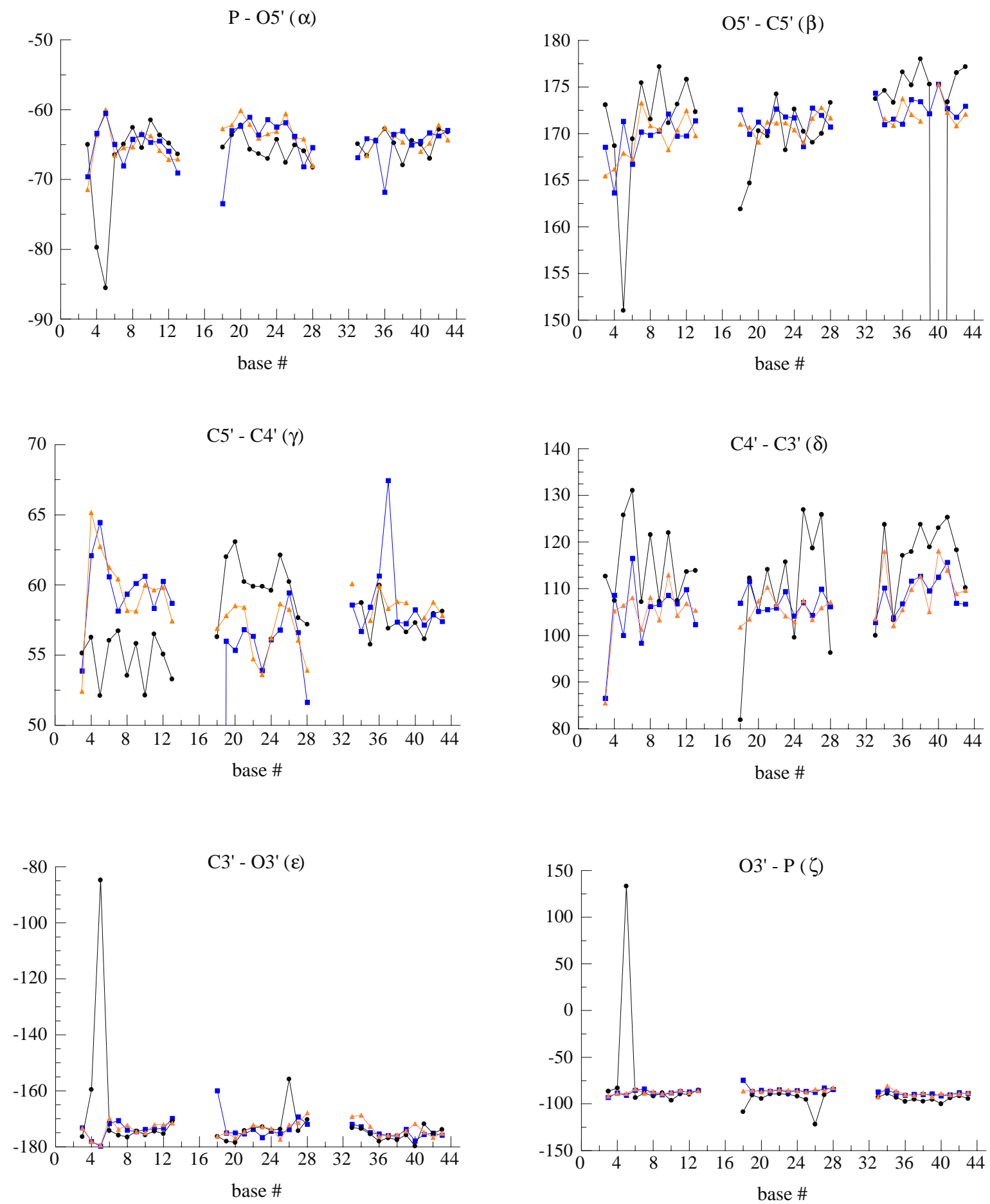

Figure 5.7. Average backbone parameters for triplex DNAs: $\mathrm{T}_{15}-\mathrm{A}_{15}: \mathrm{T}_{15}(\bullet),{ }^{5 \mathrm{sp}} \mathrm{T}_{15}-\mathrm{A}_{15}: \mathrm{T}_{15}(\square)$ and ${ }^{5 \mathrm{sp}} \mathrm{T}_{15}-\mathrm{A}_{15}: \mathrm{T}_{15}(\Delta)$. Bases 3-12 are the T-strand (* $=5 \mathrm{sp}$ or $\left.6 \mathrm{sp}\right)$, bases 19-28 are the A strand, and 32-43 are the T-strand. 
Dynamics analysis: Figures 5.8 and 5.9 show the molecular dynamics production process of double stranded and triple stranded DNAs with 6sp spin-label in a water box from $20 \mathrm{ps}$ to $1.0 \mathrm{~ns}$. The $20 \mathrm{ps}$ equilibration (data not shown), during which the system was heated from $\mathrm{T}=100 \mathrm{~K}$ to $300 \mathrm{~K}$, was reached rather rapidly, within $5 \mathrm{ps,}$, which suggests that $20 \mathrm{ps}$ is a reasonable time for the solvent to relax around solute. During the production run the temperature was kept constant $(\mathrm{T}=300 \mathrm{~K})$. These plots show that all the parameters are fluctuating near their constant values throughout all production run and indicate that there are no drastic changes in the system during the whole run. For the ds DNA (figure 5.3) one can notice rather large spike around $500 \mathrm{ps}$ for some of the parameters such as density, EPtot and EKtot. These spikes are artifacts of restarted runs at that point where the temperature was increased from $100 \mathrm{~K}$ to $300 \mathrm{~K}$ instead of having a constant value of $300 \mathrm{~K}$, and they do not affect the overall stability of a system. Volume and the pressure of the system are well stabilized and stay constant throughout the run. The data for unmodified and $5 \mathrm{sp}$ modified ds and tx DNAs are not shown, since similar behavior is also observed for these structures.

For the energetic analysis, 250 configurations at equally spaced intervals from the $1 \mathrm{~ns}$ run were used. The "gas phase" energy of the nucleic acid solute (omitting all water molecules and counterions) is computed using the modified Cornell et al. force field including all pairwise interactions. Averages of these energies represent contributions from 1) bond, angle, dihedral, which otherwise called internal energy, 2) non-bonded van der Waals, and 1-4 van der Waals energies, and 3) electrostatic and 1-4 electrostatic energies. The calculated energies for various duplex $\mathrm{A}_{15}: \mathrm{T}_{15}, \mathrm{~A}_{15}:{ }^{5 \mathrm{sp}} \mathrm{T}_{15}$ and $\mathrm{A}_{15}{ }^{6 \mathrm{sp}} \mathrm{T}_{15}$ as well as triplex $\mathrm{T}_{15}-\mathrm{A}_{15}: \mathrm{T}_{15}$, 


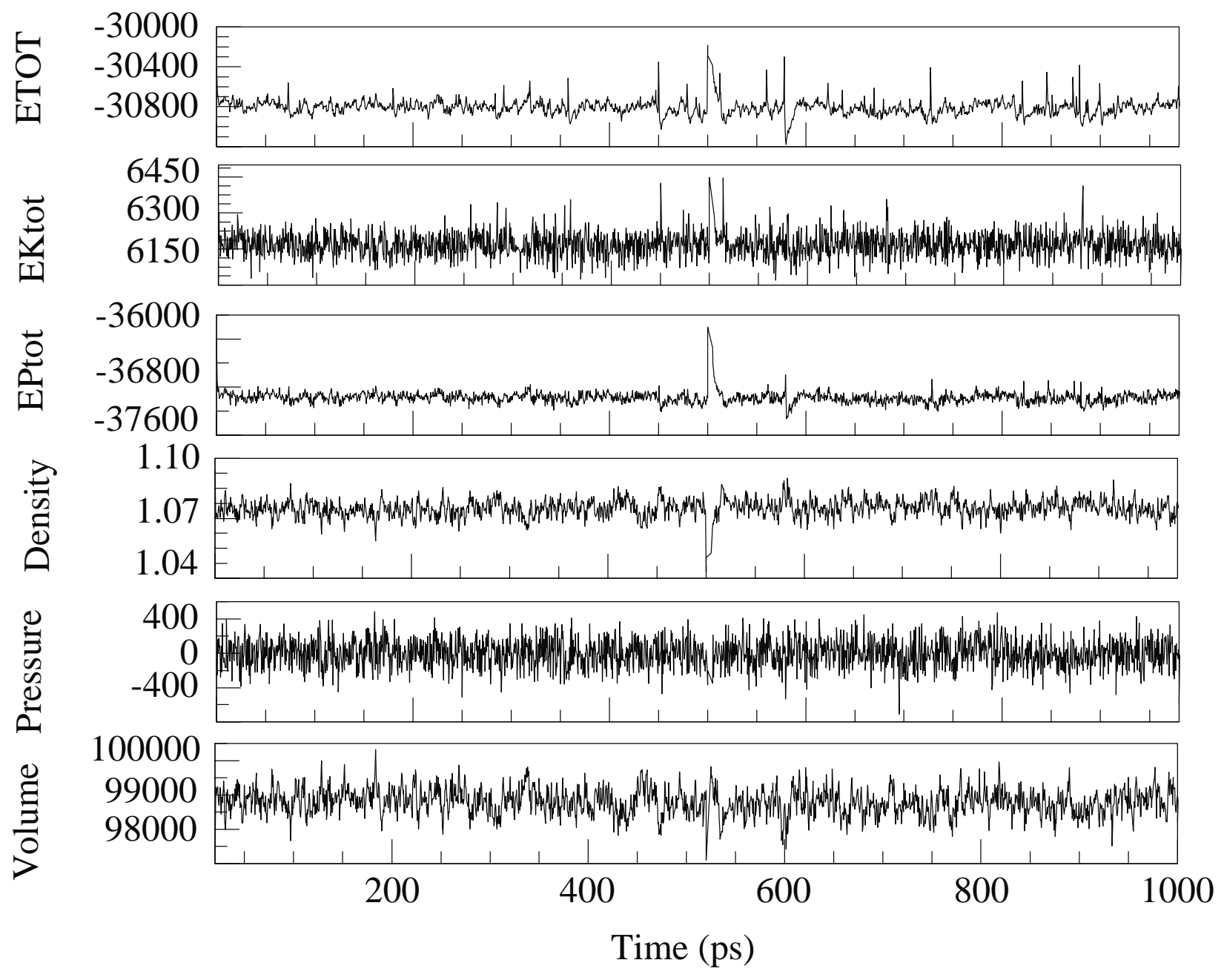

Figure 5.8. Production run for $1 \mathrm{~ns}$ of $\mathrm{A}_{15}:{ }^{6 \mathrm{sp}} \mathrm{T}_{15}$ duplex DNA. Total (ETOT), kinetic (EKtot), and potential (EPtot) energies are in $\mathrm{kcal} / \mathrm{mole}$, density is in $\mathrm{g} / \mathrm{cm}^{3}$, pressure is in atmospheres, and volume in $\AA^{3}$. 


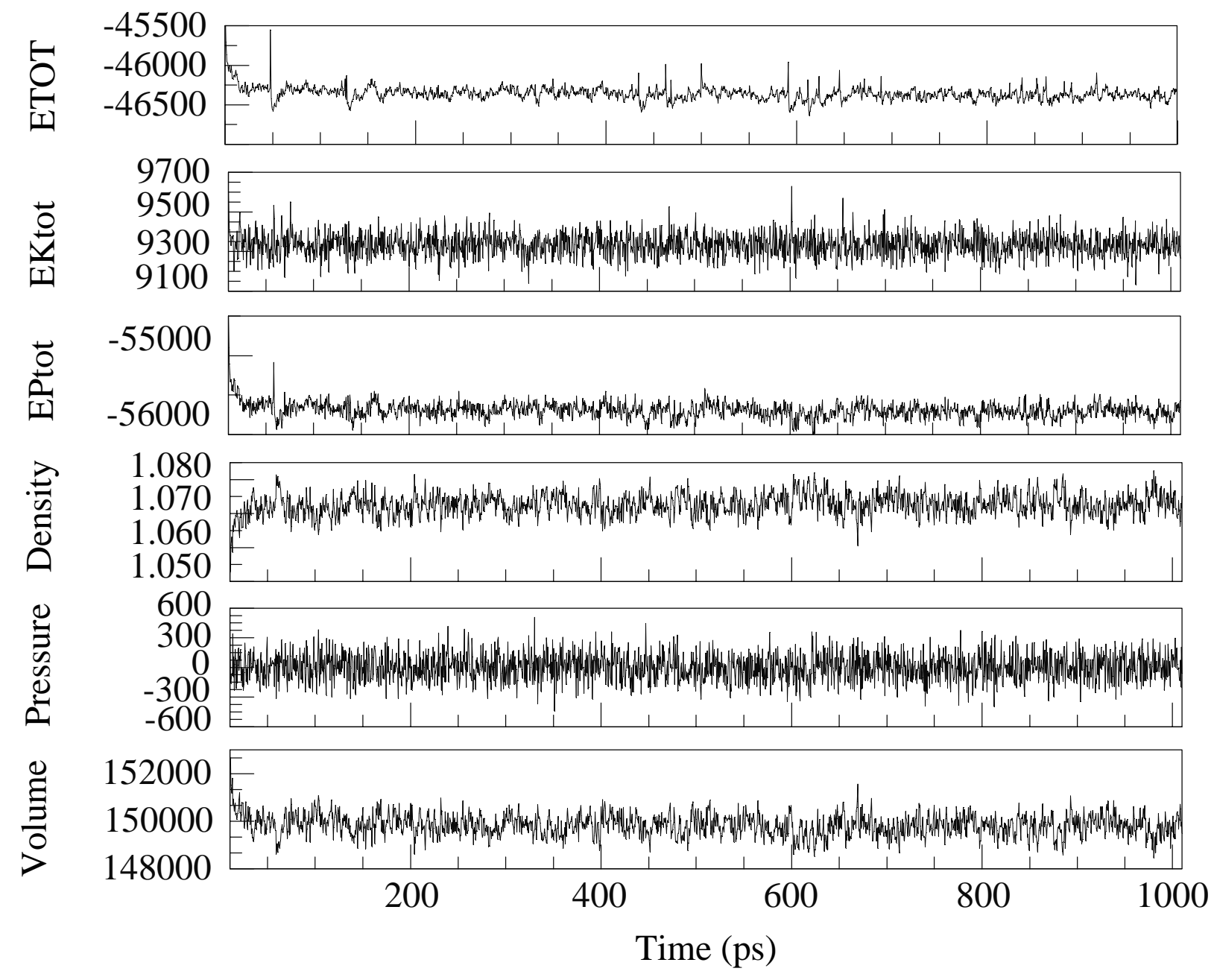

Figure 5.9. Production run for $1 \mathrm{~ns}$ of ${ }^{6 \mathrm{sp}} \mathrm{T}_{15}-\mathrm{A}_{15}: \mathrm{T}_{15}$ triplex DNA. Total (ETOT), kinetic (EKtot), and potential (EPtot) energies are in $\mathrm{kcal} / \mathrm{mole}$, density is in $\mathrm{g} / \mathrm{cm}^{3}$, pressure is in atmospheres, and volume in $\AA^{3}$. 
${ }^{5 \mathrm{sp}} \mathrm{T}_{15}-\mathrm{A}_{15}: \mathrm{T}_{15}$, and ${ }^{6 \mathrm{sp}} \mathrm{T}_{15}-\mathrm{A}_{15}: \mathrm{T}_{15}$, structures are presented in Table 5.10 along with their standard deviations shown in parenthesis. Estimation of the solvation energies for the nucleic solute was performed using Generalized Born (GB) method to calculate the electrostatic component of the solvation free energy. The total energy, $E_{t o t}$, reflects the sum of "gas phase" energy, $E_{g a s}$, and solvation free energy, $E_{G B}$.

\begin{tabular}{|c|c|c|c|c|c|c|}
\hline & ds DNA & & & tx DNA & & \\
\hline & A:T & $\mathrm{A}:^{5 \mathrm{sp}} \mathrm{T}$ & $A:{ }^{6 s p} T$ & T-A:T & ${ }^{5 \mathrm{sp}} \mathrm{T}-\mathrm{A}: \mathrm{T}$ & ${ }^{6{ }^{s p}} \mathrm{~T}-\mathrm{A}: \mathrm{T}$ \\
\hline$E_{\text {gas }}$ & $\begin{array}{l}4,159 \\
(63.4)\end{array}$ & $\begin{array}{l}4,100 \\
(48.1)\end{array}$ & $\begin{array}{l}4,265 \\
(69.3)\end{array}$ & $\begin{array}{l}10,791 \\
(77.1)\end{array}$ & $\begin{array}{l}10,619 \\
(65.1)\end{array}$ & $\begin{array}{l}10,784 \\
(89.3)\end{array}$ \\
\hline$E_{G B}$ & $\begin{array}{l}-8,798 \\
(56.0)\end{array}$ & $\begin{array}{l}-8,653 \\
(41.6)\end{array}$ & $\begin{array}{l}-8,454 \\
(43.9)\end{array}$ & $\begin{array}{l}-17,408 \\
(72.1)\end{array}$ & $\begin{array}{l}-17,150 \\
(61.4)\end{array}$ & $\begin{array}{l}-17,360 \\
(63.0)\end{array}$ \\
\hline$E_{\text {tot }}$ & $-4,637$ & $-4,554$ & $-4,189$ & $-6,617$ & $-6,531$ & $-6,575$ \\
\hline
\end{tabular}

Table 5.10. Internal energies, solvation free energies calculated with GB method, and a total energies of all duplexes and triplexes. All energies are calculated from 250 structures over 1000 ps and the units of energy are $\mathrm{kcal} / \mathrm{mol}$. Standard deviations are presented in parenthesis (italic).

Comparing overall gas energies, we can see that both double and triple $5 \mathrm{sp}$ modified DNAs have lower energies as compared to unmodified and 6sp modified duplexes and triplexes. On the other hand, solvation free energies calculated by GB method give lower energies to unmodified duplex and triplex in comparison to modified ones. This result in overall average energies of AT duplex being lower by $83 \mathrm{kcal} / \mathrm{mol}$ than the average energies of ${ }^{5 \mathrm{sp}} \mathrm{TA}$ and by $448 \mathrm{kcal} / \mathrm{mol}$ of ${ }^{6 \mathrm{sp}} \mathrm{TA}$ modified duplexes. These numbers indicate that the modification of the double stranded DNA does not improve the stability. In contrast to the 
duplexes the difference between unmodified and 5sp and 6sp modified triplexes is not that big. It is only $86 \mathrm{kcal} / \mathrm{mol}$ for TAT vs ${ }^{5 \mathrm{sp}} \mathrm{TAT}$ and $42 \mathrm{kcal} / \mathrm{mol}$ for TAT vs ${ }^{6 \mathrm{sp}} \mathrm{TAT}$. Thus we can conclude that the spin label modified triplexes may have some improved stability over the modified duplexes.

Correlation time calculation: In order to calculate correlation times from MD trajectories, the theoretical model based on the statistical random walk was used. First we define magnetic axis coordinate system of the nitroxide label, as shown in the Figure 5.10, which can be determined directly from the atomic coordinates of a MD trajectory. The probability that a particle is located between $x$ and $x+d x$ after a time $t$ can be approximated by

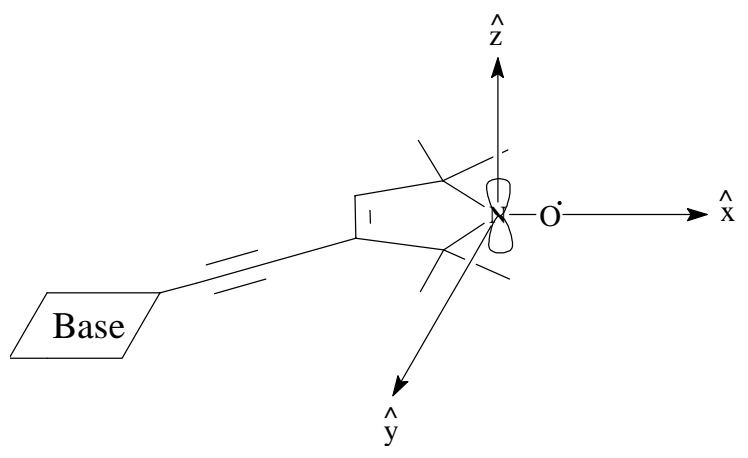

Figure 5.10. Definition of the magnetic axis coordinate system for calculation of rotational diffusion coefficients.

the Gaussian distribution function in the limit of large number of steps. Rotational diffusion coefficient in three-dimensional space, where the diffusive steps are taken with respect to the angle of rotation around a single axis, can be expressed as

$$
\boldsymbol{R}=\frac{1}{2 t} \int \boldsymbol{\theta} P(\boldsymbol{\theta}, t) \boldsymbol{\theta} d^{3} \boldsymbol{\theta}=\frac{C(\boldsymbol{\theta}, t)}{2 t}
$$


Using the MatLab program (Appendix B), the covariance matrix $C(\boldsymbol{\theta}, t)$ is calculated, diagonalized and its principle diffusion axes values are plotted with respect to time. From these plots rotational diffusion coefficients $R_{x}, R_{y}$, and $R_{z}$ are calculated, where the largest $R_{x}$ $=\mathrm{R}_{\|}$and the geometrical average of $\mathrm{R}_{\mathrm{y}}$ and $\mathrm{R}_{\mathrm{z}}=\mathrm{R}_{\perp}$. Considering $\mathrm{R}$ isotropic and axially symmetric within the nitroxide coordinate frame the rotational correlation time $\tau_{R}$, which is analogous to $\tau_{c(\text { local })}$ is given by

$$
\tau_{c(\text { local })}=\frac{1}{6 \sqrt{R_{\perp} R_{\|}}}
$$

In our case $\mathrm{R}$ is not isotropic, which leads to the anisotropic rotational correlation time $\tau_{R}$. Thus, it is better to divide the correlation time into its two components $\tau_{\perp}$ and $\tau_{\|}$, and they can be calculated as

$$
\tau_{\perp}=\frac{1}{6 R_{\perp}}, \quad \tau_{\|}=\frac{1}{6 R_{\|}}
$$

These calculated values along with rotational diffusion coefficients are summarized in the Table 5.11. As expected, the correlation time of the spin label attached to the triplex DNA is slower than the corresponding time of the label in the duplex. These differences are not very large, but they do suggest that the spin label is more constrained in the triplex than in the duplex. This finding also correlates with the correlation time obtained from simulation of EPR data. Though the time scale is different. Which brings to another conclusion that the EPR spectra are mostly affected by global tumbling of the DNA and that the contribution of the spin label motion is small. 


\begin{tabular}{|c|c|c|c|c|c|c|}
\hline & & ${ }^{5 \mathrm{sp}} \mathbf{T}$ & $A:^{5 \mathrm{sp}} \mathrm{T}$ & ${ }^{{ }^{s s p}} \mathrm{~T}-\mathrm{A}: \mathrm{T}$ & $A:^{6 s p} T$ & ${ }^{6 s p} \mathrm{~T}-\mathrm{A}: \mathrm{T}$ \\
\hline $\mathrm{R}_{\mathrm{y}}$ & \multirow{2}{*}{$\mathrm{R}_{\perp}$} & 3.88 & 3.17 & 2.95 & 3.64 & 3.23 \\
\hline $\mathrm{R}_{\mathrm{z}}$ & & 6.10 & 6.56 & 5.28 & 7.44 & 7.57 \\
\hline$\overline{R_{x}}$ & $\mathrm{R}_{\|}$ & 15.54 & 20.63 & 15.12 & 26.15 & 20.48 \\
\hline \multicolumn{2}{|l|}{$\tau_{\perp}$} & 3.34 & $3.42 \mathrm{ps}$ & $4.06 \mathrm{ps}$ & $3.01 \mathrm{ps}$ & $3.86 \mathrm{ps}$ \\
\hline \multicolumn{2}{|l|}{$\tau_{\|}$} & $1.07 \mathrm{ps}$ & $0.81 \mathrm{ps}$ & $1.10 \mathrm{ps}$ & $0.64 \mathrm{ps}$ & $0.81 \mathrm{ps}$ \\
\hline
\end{tabular}

Table 5.11. Rotational diffusion coefficients and correlation times calculated for $5 \mathrm{sp}$ modified ss DNA, and $5 \mathrm{sp}$ and $6 \mathrm{sp}$ modified ds and tx DNAs. Diffusion coefficients are in $10^{10} \mathrm{~s}^{-1}$. 
Single stranded DNA analysis: A special case is represented by the single stranded DNA dynamics. As mentioned earlier, the range of accessible conformations are dependent on environmental factors such as explicit solvent or counterions representation. The dynamic nature of a double stranded DNA is reduced relative to ss DNA because of the hydrogen bonding between complementary strands. For the single stranded DNA such interactions are absent, which makes the molecule more flexible with more degrees of freedom. Several studies have been conducted on studying the interaction of metal ions with single stranded nucleotides to show the importance of counterions on the dynamics and structure of the $\operatorname{stand}^{50}$. So far no studies have been conducted on ss DNAs with covalently attached spin labels. Here we show the influence of such a modification on the global conformation of ss DNA helix.

Following the backbone dynamics for unmodified ss DNA we do not see any substantial deformation of the chain (data not shown) during the course of the simulation. Referring to the average values for backbone torsional parameters for canonical DNA structures are summarized in the table 5.12 and all the angles of the backbone fall into the average defined range. In contrast, looking at the backbone dynamics of 5sp modified ss DNA we notice large changes in several backbone angles during the whole simulation. Selected angles that show anomalous behavior are plotted in the Figure 5.11 as a function of time. The torsional angle $\alpha$ of the $5^{\text {th }}$ base starts changing its average value at about $350 \mathrm{ps}$ (Figure 5.11a). We can observe rather sharp jump from $-50^{\circ}$ to approximately $50^{\circ}$ is at 550 ps. Similar behavior is detected for the torsional angle $\gamma$ of the $5^{\text {th }}$ base. Walking down to the $7^{\text {th }}$ base of the strand we look at the angle $\xi$ (Figure 5.11b). Here, the conformational change 
A

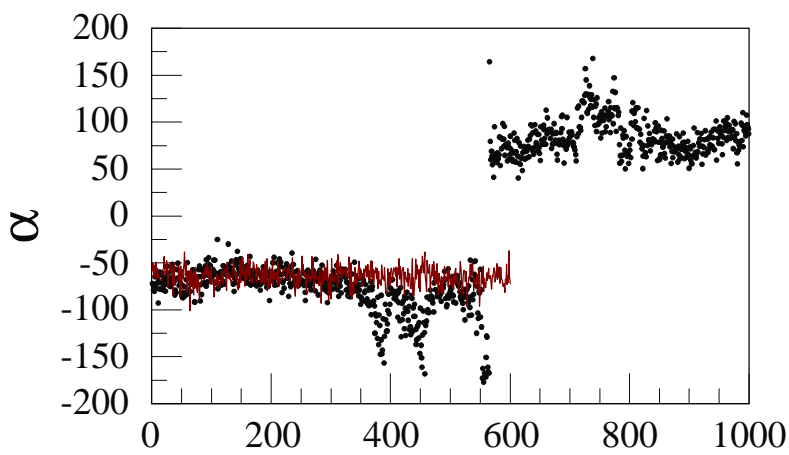

Time (ps)

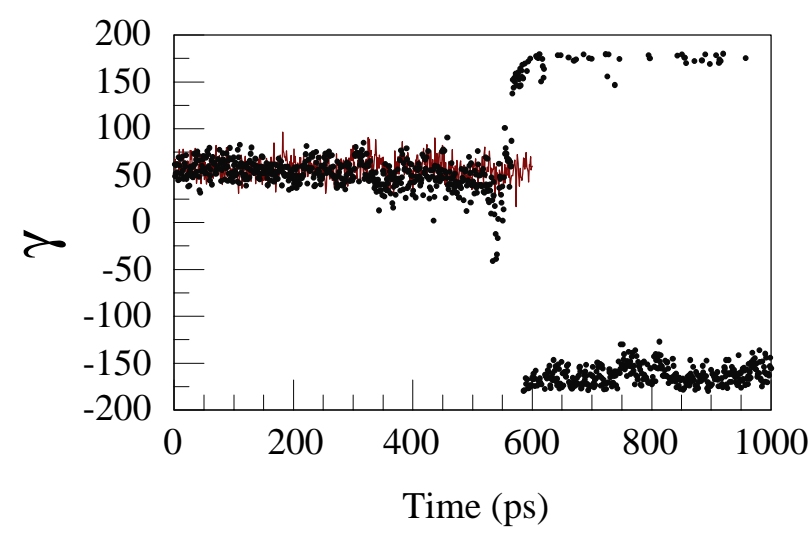

B

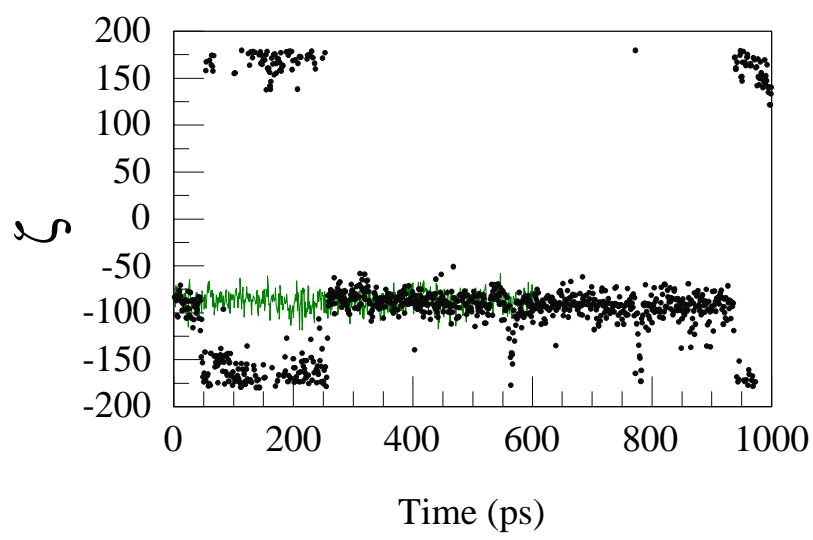

Figure 5.11. Selected backbone torsional parameters for $5 \mathrm{sp}$ modified ss DNA. A) and of the $5^{\text {th }}$ base, B) of the $7^{\text {th }}$ base. Dotted plot represents unconstrained dynamics over $1 \mathrm{~ns}$, solid line represents constrained dynamics over 650 ps run. 
happens at a quite early stage of the dynamics run, around $50 \mathrm{ps}$. This indicates that the presence of the spin label invokes large conformational changes in the single strand and these changes are repeated over the entire simulation. These conformational changes are promoted by the stacking of the acetylenic tether with the adjacent bases up and down the strand. Besides the acetylenic tether, the nitroxide ring is also involved in the stacking interaction with other bases. By looking at the selected configurations of the ss DNA from the entire run (Figure 5.12) the changes in the backbone conformation of the strand become evident. Also, comparing the global axis parameters we notice a substantial change in the curvature of the 5sp modified $\mathrm{T}$ strand with respect to unmodified one (Table 5.12). These changes in the curvature result in the overall bending of the strand axis.

\begin{tabular}{|c|c|c|}
\hline & $T$ & ${ }^{5 s p} T$ \\
\hline Curvature & & \\
\hline Path length & 50.01 & 55.70 \\
\hline End-to-end & 49.37 & 36.86 \\
\hline Shortening & $1.23 \%$ & $33.82 \%$ \\
\hline & $\mathrm{UU}=8.01$ & $\mathrm{UU}=27.99$ \\
Overall axis bend & $\mathrm{PP}=9.27$ & $\mathrm{PP}=53.27$ \\
& & \\
\hline
\end{tabular}

Table 5.12. Global axis parameters for unmodified and 5 sp modified ss DNA. 


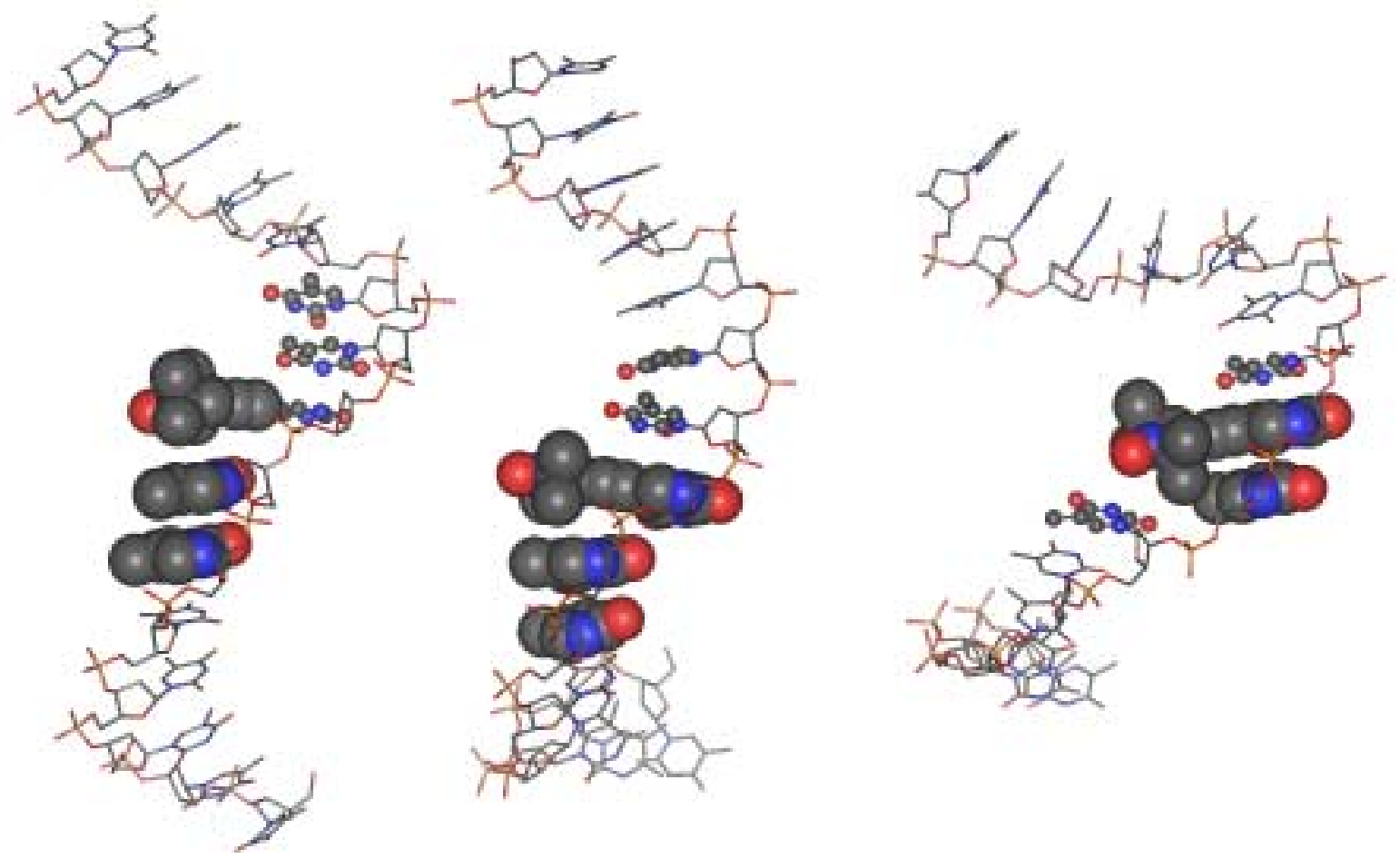

Figure 5.12. $5 \mathrm{sp}$ modified ss DNA snapshots at different points during the $1 \mathrm{~ns}$ run. 


\section{Conclusions}

The synthesis of a new spin label nitroxide in a 6-membered ring, which is rigidly attached to a uridine, has been described. This new spin labeled DNA base, is synthetically simpler to prepare than the previous 5-membered ring analog, and at the same time, provides comparable yields during automated DNA synthesis and similar EPR data. Moreover, this spin label has been used to detect a triplex DNA as well double stranded DNA. The EPR data suggest that the triplex DNA is slightly less mobile than double stranded DNA though this may be due to the increase in size of the triplex DNA relative to the double stranded DNA.

Molecular modeling studies of both 5/6sp modified and unmodified DNAs have shown that the spin label induces conformational changes in both duplexes and triplexes. In the duplexes this conformational change result in the structure which tends to be more ADNA like. To the less extent these changes can be seen in the modified triplexes, since they are more rigid and will require longer simulations in order to achieve noticeable change in the conformation. Correlation time of the nitroxide extracted from MD trajectories indicates slower rotation of the spin label in the triplex than in the duplex as it was shown from EPR data. As for $5 \mathrm{sp}$ modified ss DNA the correlation time came out to be slower than in the duplex, which did not agree with the EPR data. This discrepancy happens to be a result of the altered strand geometry because of the stacking interaction of the nitroxide moiety and the acetylenic tether with adjacent bases.

For the future work 1) constrained MD need to be done for modified ss DNAs, 2) high field EPR data needs to be obtained in order to get correlation times reflecting the motion of the spin label, and to relate these values to the correlation times obtained from MD trajectories. 


\section{References}

1. Felsenfeld, G.; Davies, D. R.; Rich, A. J. Am. Chem. Soc. 79, 2023-2024, (1957).

2. Mirkin, S. M.; Lyamichev, V. I.; Drushlyak, K. N.; Dobrynin, V. N.; Filippov, S. A.; Frank-Kamenetskii, M. D. Nature 330, 495-497, (1987).

3. Dayn, A.; Samadashwily, G. M.; Mirkin, S. M. Proc. Natl. Acad. Sci. USA 89, 1140611410, (1992).

4. Frank-Kamenetskii, M. D.; Mirkin, S. M. Annu. Rev. Biochem. 64, 65-95, (1995).

5. Soliva, R.; Garcia, R. G.; Blas, J. R.; Eritja, R.; Asensio, J. L.; Gonzalez, C.; Luque, F. J.; Orozco, M. Nucl. Acids Res. 28, 4531-4539, (2000).

6. Steinhoff, H.-J.; Hubbell, W. L. Biophysical Journal 71, 2201-2212, (1996).

7. Keyes Robert S.; Cao, Y. Y.; Bobst, E. V.; Rosenberg, J. M.; Bobst Albert M. Journal of Biomolecular Structure \& Dynamics 14, 163-172, (1996).

8. Kao, S.-C.; Bobst, A. M. Biochemistry 24, 5465-5469, (1985).

9. Edwards, T. E.; Okonogi, T. M.; Robinson, B. H.; Sigurdsson, S. Th. J. Am. Chem. Soc. 123, 1528, (2001).

10. Pauly, G. T.; Thomas, I. E.; Bobst., A. M. Biochemistry 26, 7304-7310, (1987).

11. Strobel, O. K.; Kryak, D. D.; Bobst, E. V.; Bobst Albert M. Bioconjugate Chem. 2, 8995, (1991).

12. Keyes, R. S.; Bobst, A. M. Biophysical Chemistry 45, 281-303, (1993).

13. Strobel, O. K.; Keyes Robert S.; Sinden, R. R.; Bobst Albert M. Arch. Biochem. Biophys. 324, 357-366, (1995).

14. Keyes Robert S.; Bobst, E. V.; Cao, Y. Y.; Bobst Albert M. Biophysical Journal 72, 282-290, (1997).

15. Budil, D. E.; Lee, S.; Saxena, S.; Freed Jack H. Journal of Magnetic Resonance Series A 120, 155-189, (1996).

16. Liang Zhichun; Freed Jack H.; Keyes Robert S.; Bobst Albert M. J. Phys. Chem. B 104, 5372-5381, (2000).

17. Qin, P. Z.; Butcher, S. E.; Feigon, J.; Hubbell, W. L. Biochemistry 40, 6936, (2001).

18. Spaltenstein, A.; Robinson, B. H.; Hopkins, P. B. Biochemistry 28, 9484-9495, (1989). 
19. Spaltenstein, A.; Robinson, B. H.; Hopkins, P. B. JACS 111, 2303-2305, (1989).

20. Heystek, L. E.; Zhou, H.; Dande, P.; Gold, B. J. Am. Chem. Soc. 120, 12165-12166, (1998).

21. Colocci, N.; Dervan, P. B. J. Am. Chem. Soc. 116, 785-786, (1994).

22. Gannett, P. M.; Darian, E.; Powell, J. H.; Johnson, M. E. Synthetic Communications 31, 2137-2141, (2001).

23. Lankas, F.; Sponer, J.; Hobza, P.; Langowski, J. J. Mol. Biol. 299, 695-709, (2000).

24. Asensio, J. L.; Carr, R.; Brown, T.; Lane, A. N. J. Am. Chem. Soc. 121, 11063-11070, (1999).

25. Weiner, S. J.; Kollman, P. A.; Case, D. A.; Singh, U. C.; Ghio, C.; Alagona, G.;

Profeta, S. Jr.; Weiner, P. J. Am. Chem. Soc. 106, 765-784, (1984).

26. Brooks, B. R.; Karplus, M.; Olafson, B. D.; States, D. J.; Swaminathan, S.; Karplus, M. J. Comp. Chem 4, 187-217, (1983).

27. Nilsson, L.; Karplus, M. J. Comp. Chem 7, 591-616, (1986).

28. Mohan, V.; Cheng, Y. K.; Marlow, E.; Pettitt, B. M. Biopolymers 1317-1325, (1993).

29. Eriksson, M.; Nielsen, P. E. Quarterly Reviews of Biophysics 29, 369-394, (1996).

30. Ninaber, A.; Goodfellow, J. M. Journal of Biomolecular Structure \& Dynamics 16, 651-661, (1998).

31. CD of Nucleic Acids. In Circular Dichroism: Principles and Applications. 2nd Edition; Berova, N., Nakanishi, K., Woody, R. W., Eds.; A John Wiley \& Sons, Inc.: New York, Chichester, Weinheim, Brisbane, Singapore, Toronto, 2000; Chapter 24, 25.

32. Circular Dichroism: An Introduction. In Circular Dichroism: Principles and Applications. 2nd Edition; Berova, N., Nakanishi, K., Woody, R. W., Eds.; A John Wiley \& Sons, Inc.: New York, Chichester, Weinheim, Brisbane, Singapore, Toronto, 2000; Chapter 1.

33. Poole, C. P. Jr. In Electron Spin Resonance: A Comprehensive Treaties on Experimental Techniques; Dover Publications Inc.: Mineola, New York, 1996.

34. Weil, J. A.; Bolton, J. R.; Wertz, J. E. In Electron Pramagnetic Resonance; JohnWiley \& Sons,Inc: 1994.

35. Orton, J. W. In Electron Pramagnetic Resonance; London Iliffe Books Ltd.: 1968.

36. Martin, R. E.; Pannier, M.; Diederich, F.; Gramlich, V.; Hubrich, M.; Spiess, H. W. Angew. Chem. , Int. Ed. 37, 2834-2837, (1998). 
37. Snyder, C. H.; Soto, A. R. JOC 29, 742-747, (1964).

38. Ozinskas, A.; Bobst, A. M. Helv. Chim. Acta 63, 1407-1411, (1980).

39. Rozantsev, E. G.; and Sholle, V. D. Synthesis 401-414, (1971).

40. Chan, S. S.; Breslauer, K. J.; Hogan, M. E.; Kessl. Biochemistry 29, 6161-6171, (1990).

41. Singleton, S. F.; Dervan, P. B. Biochem. 32, 13171-13179, (1993).

42. Colocci, N.; Distefano, M. D.; Dervan, P. B. J. Am. Chem. Soc. 115, 4468-4473, (1993).

43. Allen, M. P.; Tildesley, D. J. Computer Simulation of Liquids; Oxford University Press: Oxford, 1987.

44. Brooks, C. L. I.; Karplus, M.; Pettitt, B. M. Proteins. A Theoretial Perspective of Dynamics, Structure, and Thermodynamics. In Advances in Chemical Physics; Prigogine, I., Rice, S. A., Eds.; John Wiley \& Sons: new York, 1988; Chapter 71.

45. Beveridge, D. L.; Ravishankar, G. Cur. Opt. Struct. Biol. 4, 246-255, (1994).

46. Cheatham, T. E. I.; Kollman, P. A. J. Mol. Biol. 259, 434-444, (1996).

47. Cheatham, T. E. I.; Miller, J. L.; Fox, T.; Darden, T. A.; Kollman, P. A. J. Am. Chem. Soc. 117, 4193-4194, (1995).

48. Barone, V.; Bencini, A.; Cossi, M.; Di Matteo, A.; Mattesini, M.; Totti, F. J. Am. Chem. Soc. 120, 7069-7078, (1998).

49. Stofer, E.; Lavery, R. Biopolymers 34, 337-346, (1994).

50. Martinez, J. M.; Elmroth, S. K. C.; Kloo, L. J. Am. Chem. Soc. 123, 12279-12289, (2001).

51. Fossella, J. A.; Kim, Y. J.; Shih, H.; Richards, E. G.; Fresco, J. R. Nucl. Acids Res. 21, 4511-4515, (1993). 
Appendix A

Modified parameters for nitroxides

\begin{tabular}{|c|c|c|}
\hline MASS & a.u. & \\
\hline $\mathrm{C} 1$ & 12.01 & \\
\hline LP & 0.0001 & \\
\hline NR & 14.01 & \\
\hline OR & 16.00 & \\
\hline BOND & $K_{r} \mathrm{kcal} /\left(\mathrm{mole} \cdot \AA^{2}\right)$ & $r_{e q}(\AA)$ \\
\hline C1-C1 & 700 & 1.204 \\
\hline C1-HA & 50.0 & 1.056 \\
\hline C1-CM & 600.0 & 1.440 \\
\hline CA-NR & 500.0 & 1.35 \\
\hline CT-NR & 450.1 & 1.47 \\
\hline LP-OR & 325.0 & 0.50 \\
\hline NR-OR & 337.0 & 1.28 \\
\hline ANGLE & $K_{\theta} \mathrm{kcal} /\left(\mathrm{mole} \cdot \mathrm{rad}^{2}\right)$ & $\theta_{e q}($ degree $)$ \\
\hline C1-C1-HA & 32.8 & 180.0 \\
\hline C1-C1-CM & 65.7 & 180.0 \\
\hline C1-CM-CM & 39.4 & 120.0 \\
\hline C1-CM-CT & 39.4 & 120.0 \\
\hline CM-CT-CT & 29.6 & 109.5 \\
\hline CT-CM-HA & 19.7 & 120.0 \\
\hline NR-CT-CT & 80.0 & 105.6 \\
\hline CT-NR-CT & 46.6 & 120.0 \\
\hline OR-NR-CT & 80.2 & 119.3 \\
\hline HC-CT-NR & 50.0 & 109.5 \\
\hline NR-CA-NR & 50.4 & 122.0 \\
\hline NR-CT-CM & 25.2 & 100.1 \\
\hline CA-NR-OR & 50.8 & 124.0 \\
\hline CA-NR-CT & 25.2 & 122.0 \\
\hline LP-OR-LP & 80.0 & 120.0 \\
\hline LP-OR-NR & 80.0 & 120.0 \\
\hline
\end{tabular}




\begin{tabular}{|c|c|c|c|c|}
\hline DIHEDRAL & \# of paths & ${ }^{2} V_{n} / 2$ & ${ }^{3} \gamma$ & ${ }^{4} n^{I}$ \\
\hline $\mathrm{X}-\mathrm{C} 1-\mathrm{C} 1-\mathrm{X}$ & 1 & 0.25 & 180.0 & 1. \\
\hline $\mathrm{X}-\mathrm{CM}-\mathrm{C} 1-\mathrm{X}$ & 2 & 0.0 & 180.0 & 1. \\
\hline NT-CT-CT-X & 9 & 0.2 & 0.0 & 3. \\
\hline X -NR-CT-X & 6 & 0.3 & 0.0 & 2. \\
\hline X -NR-CA-X & 6 & 6.3 & 0.0 & 2. \\
\hline X -NR-OR-X & 4 & 1.0 & 0.0 & 2. \\
\hline OOP & & & & \\
\hline NR-CT-CT-OR & - & 4.0 & 180.0 & 2. \\
\hline X -X -NR-OR & - & 10.1 & 180.0 & 2. \\
\hline X -X -NR-CT & - & 1.2 & 180.0 & 2. \\
\hline NONB & \multicolumn{2}{|c|}{${ }^{5} R *(\AA)$} & \multicolumn{2}{|c|}{${ }^{6} \varepsilon^{*}(\mathrm{kcal} / \mathrm{mole})$} \\
\hline $\mathrm{C} 1$ & \multicolumn{2}{|c|}{1.908} & \multicolumn{2}{|c|}{0.086} \\
\hline NR & \multicolumn{2}{|c|}{3.66} & \multicolumn{2}{|c|}{0.069} \\
\hline OR & \multicolumn{2}{|c|}{0.00} & \multicolumn{2}{|c|}{0.060} \\
\hline LP & \multicolumn{2}{|c|}{0.00} & \multicolumn{2}{|c|}{0.061} \\
\hline
\end{tabular}

\footnotetext{
${ }^{1}$ \# of bond paths that the total $V_{n} / 2$ is divided into. This is equal to the product of the \# of the bonds to each of the middle two atoms.

${ }^{2}$ Magnitude of torsion in $\mathrm{kcal} / \mathrm{mole}$.

${ }^{3}$ Phase offset in degree.

${ }^{4}$ Periodicity of the torsion.

${ }^{5}$ Van der Waals radius for given atom $R_{i j} *=\left(R_{i i} * R_{j j} *\right)^{1 / 2}$.

${ }^{6}$ Van der Waals well depth for given atom. $\varepsilon_{i j} *=\left(\varepsilon_{i i} * \varepsilon_{j j}{ }^{*}\right)^{1 / 2}$
} 


\section{Appendix B}

\section{Matlab script}

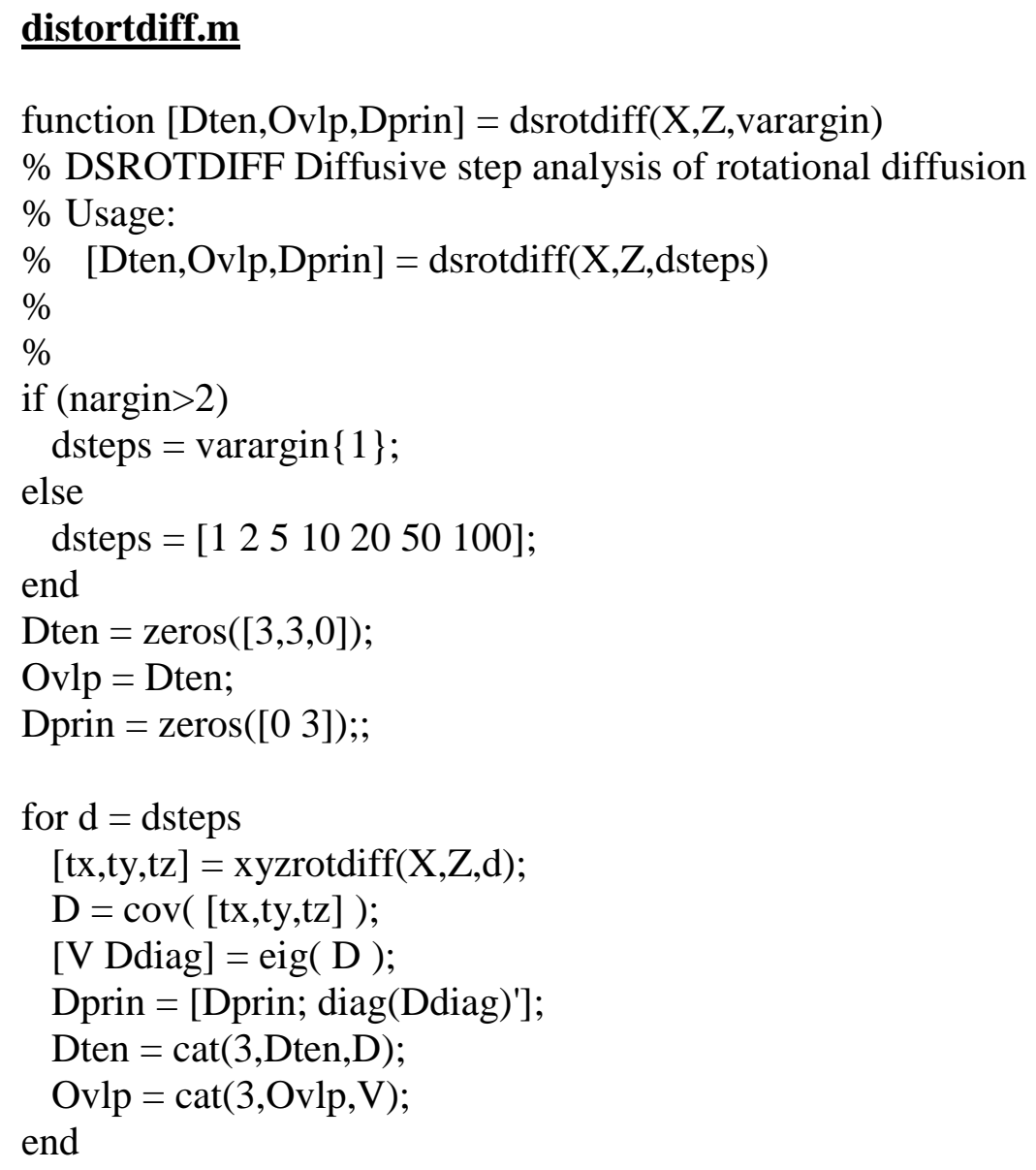

\section{inpgdata.m}

function $[X, Z]=$ inpgdata

$\%$

$\%$ Load in AMBER calculateion results from Peter Gannett's lab

$\%$

pgdir $=[$ 'c: $\backslash$ EdarlMatLab_splat6m_duplex_vectors $\backslash$ '] $\%$ Set this to directory where data is stored

$\% \quad$ Used to be: 'c:Iwindows\profiles ldavid budillmy documents

$\mathrm{NO}=\operatorname{load}([$ pgdir, 'NOvector.tbl']);

$\mathrm{NC1}=\operatorname{load}([$ pgdir,'NC16vector.tbl']);

NC2 = load([pgdir,'C14Nvector.tbl']); 
rownorm = inline('M./kron(sqrt(sum(M.^2,2)),ones(1,size(M,2)))','M');

$\mathrm{X}=\operatorname{rownorm}(\mathrm{NO})$;

$\mathrm{Z}=\operatorname{rownorm}(\operatorname{cross}(\mathrm{NC} 1, \mathrm{NO})+\operatorname{cross}(\mathrm{NC} 2, \mathrm{NO}))$;

\section{diffdistr.m}

function diffdistr(X,Z,varargin)

$\left[\right.$ tx ty tz] = xyzrotdiff $\left(X, Z, \operatorname{varargin}^{51}\right)$;

$\mathrm{C}=\operatorname{cov}([\mathrm{tx}$ ty tz $])$

$[\mathrm{V}, \mathrm{D}]=\operatorname{eig}(\mathrm{C})$;

figure(1);

$[\mathrm{nx}, \mathrm{xx}]=\operatorname{hist}(\mathrm{tx}, 101)$;

$[\mathrm{ny}, \mathrm{xy}]=\operatorname{hist}(\mathrm{ty}, 101)$;

$[\mathrm{nz}, \mathrm{xz}]=\operatorname{hist}(\mathrm{tz}, 101)$;

$\operatorname{subplot}(3,2,1)$

$\operatorname{bar}(\mathrm{xx}, \mathrm{nx})$;

$\operatorname{axis}([-\max (\operatorname{abs}(x x)) \max (\operatorname{abs}(x x)) 0 \max (n x)])$;

ylabel('n (theta_x)')

subplot( $(3,2,3)$;

bar(xy,ny);

$\operatorname{axis}([-\max (\operatorname{abs}(x y)) \max (\operatorname{abs}(x y)) 0 \max (n y)])$;

ylabel('n (theta_y)')

subplot $(3,2,5)$

bar(xz,nz);

$\operatorname{axis}([-\max (\operatorname{abs}(\mathrm{xz})) \max (\operatorname{abs}(\mathrm{xz})) 0 \max (\mathrm{nz})])$;

xlabel ('Rotation angle (rad)');

ylabel('n (theta_z)')

subplot(3,2,2);

plot(tx,ty,'.');

xlabel('theta_x');

ylabel('theta_y')

$\operatorname{subplot}(3,2,4)$

plot(ty,tz,'.');

xlabel('theta_y');

ylabel('theta_z')

subplot(3,2,6); 
plot(tx,tz,'.');

xlabel('theta_x');

ylabel('theta_z');

\section{pmgworkup.m}

$\%$ Macro file to construct angle distribution plots and

$\%$ calculation of diffusion constant from variance

$\%$

$\%$

[X,Z] = inpgdata;

dsteps = $\left[\begin{array}{lllllll}1 & 2 & 5 & 10 & 20 & 50 & 100\end{array}\right] ; \quad \%$ Set list of time steps to be utilized

[Dten,Ovlp,Dprin] = dsrotdiff(X,Z,dsteps); \% Calculate diffusion tensors for each time step

[Dsort,ix] $=\operatorname{sort}($ Dprin, 2$) ; \quad \%$ sort eigenvalues

$\%$

$\%$ Plot distribution of diffusion angles (distribution of theta_x,y,z and correlations)

$\%$

figure(1);

diffdistr(X,Z,50);

figure(2)

$\operatorname{plot}([\mathrm{dsteps}$, Dsort],'o');

zoom on;

$\%$

$\%$ Define range of indices into dsteps vector identifying which steps will be

$\%$ used in linear fit to calculate diffusion tensor

range $=[3: 7]$

$\mathrm{d}=\operatorname{dsteps}([\operatorname{range}(1)$, range $($ end $)])$;

$\%$

$\%$ Fit lines to variance of angle for each principal direction with time

line $1=\operatorname{polyfit}\left(\operatorname{dsteps}(\text { range })^{\prime}\right.$, Dsort $($ range, 1$\left.), 1\right)$;

line2 $=\operatorname{polyfit}($ dsteps $($ range $)$ ',Dsort $($ range, 2$), 1)$;

line3 = polyfit $($ dsteps $($ range $) '$, Dsort $($ range, 3$), 1)$;

$\%$

$\%$ Plot lines on graph

line(d, polyval(line1,d) );

line(d, polyval(line 2,d) );

line $(\mathrm{d}$, polyval $($ line $3, \mathrm{~d}))$;

ylabel('Variance of angle/ $\mathrm{rad}^{\wedge} 2$ ');

xlabel('time/ps');

$\%$

$\%$ Display resulting diffusion coefficients in command window $\operatorname{disp}([$ 'D1 =',num2str(line1(2)/2e-12)]); 
$\operatorname{disp}([$ 'D2 =',num2str(line2(2)/2e-12)]);

$\operatorname{disp}([$ 'D3 =',num2str(line3(2)/2e-12)]);

\section{xyzrotdiff.m}

function $[\mathrm{tx}, \mathrm{ty}, \mathrm{tz}]=\operatorname{xyzrotdiff}(\mathrm{X}, \mathrm{Z}, \operatorname{varargin})$;

$\%$

$\%$ XYZROTDIFF Carry out diffusive step analysis on a nitroxide trajectory

$\%$

$\%$ Usage: $[\mathrm{tx}, \mathrm{ty}, \mathrm{tz}]=\operatorname{diffstep}(\mathrm{X}, \mathrm{Z}, \mathrm{d})$

$\%$

\% Inputs: $\mathrm{X}$ Trajectory matrix for $\mathrm{X}$ axis (cols: $\mathrm{x}, \mathrm{y}, \mathrm{Z}$ coords; each row is $\mathrm{pt}$ in trajectory)

$\% \quad \mathrm{Z}$ Trajectory matrix for $\mathrm{Z}$ axis (cols: $\mathrm{x}, \mathrm{y}, \mathrm{z}$ coords; each row is pt in trajectory)

$\% \quad$ d Trajectory step size for diffusion calculation (default 1 )

$\%$

$\%$

$\%$ Outputs: tx Vector of theta_x values for the trajectory

$\%$ ty Vector of theta_y values for the trajectory

$\%$ tz Vector of theta_z values for the trajectory

$\%$

if nargin $>2$

$\mathrm{d}=\operatorname{varargin}\{1\}$;

else

$\mathrm{d}=1$;

end

$\%$

$\%$ Form an $\mathrm{R}$ matrix for each point in the trajectory (calculate $\mathrm{Y}$ column as ZxX)

$\%$

ntraj = length $(\mathrm{X})$;

$\mathrm{R}=\operatorname{reshape}([\mathrm{X}, \operatorname{cross}(\mathrm{Z}, \mathrm{X}), \mathrm{Z}]$ ', [3 3 ntraj] $)$;

$\%$

$\%$ Build a trajectory of difference rotation matrices for the given step difference

$\%$

$\mathrm{dR}=\operatorname{zeros}(3,3,0)$;

for ix $=1$ :ntraj-d

$\mathrm{dR}=\operatorname{cat}\left(3, \mathrm{dR}, \mathrm{R}(:, ;, \mathrm{ix})^{\prime} * \mathrm{R}(:, ;, \mathrm{ix}+\mathrm{d})\right)$;

end

$\%$

$\%$ Determine the theta_x, theta_y, and theta_z angles for each difference rotation matrix $\%$

$\operatorname{tx}=\operatorname{atan}($ squeeze $(\mathrm{dR}(2,3,:) \cdot / \mathrm{dR}(3,3,:)))$;

ty $=\operatorname{asin}(\operatorname{squeeze}(\mathrm{dR}(1,3,:)))$

$\mathrm{tz}=\operatorname{atan}(\operatorname{squeeze}(\mathrm{dR}(1,2,:) \cdot / \mathrm{dR}(1,1,:))) ;$ 